TRANSACTIONS OF THE

AMERICAN MATHEMATICAL SOCIETY

Volume 351, Number 8, Pages 3073-3117

$\mathrm{S}$ 0002-9947(99)02145-5

Article electronically published on April 8, 1999

\title{
FORCING MINIMAL EXTENSIONS OF BOOLEAN ALGEBRAS
}

\author{
PIOTR KOSZMIDER
}

\begin{abstract}
We employ a forcing approach to extending Boolean algebras. A link between some forcings and some cardinal functions on Boolean algebras is found and exploited. We find the following applications:

1) We make Fedorchuk's method more flexible, obtaining, for every cardinal $\lambda$ of uncountable cofinality, a consistent example of a Boolean algebra $A_{\lambda}$ whose every infinite homomorphic image is of cardinality $\lambda$ and has a countable dense subalgebra (i.e., its Stone space is a compact S-space whose every infinite closed subspace has weight $\lambda$ ). In particular this construction shows that it is consistent that the minimal character of a nonprincipal ultrafilter in a homomorphic image of an algebra $A$ can be strictly less than the minimal size of a homomorphic image of $A$, answering a question of J. D. Monk.

2) We prove that for every cardinal of uncountable cofinality it is consistent that $2^{\omega}=\lambda$ and both $A_{\lambda}$ and $A_{\omega_{1}}$ exist.

3) By combining these algebras we obtain many examples that answer questions of J.D. Monk.

4) We prove the consistency of $\mathrm{MA}+\neg \mathrm{CH}+$ there is a countably tight compact space without a point of countable character, complementing results of A. Dow, V. Malykhin, and I. Juhasz. Although the algebra of clopen sets of the above space has no ultrafilter which is countably generated, it is a subalgebra of an algebra all of whose ultrafilters are countably generated. This proves, answering a question of Arhangel'skii, that it is consistent that there is a first countable compact space which has a continuous image without a point of countable character.

5 ) We prove that for any cardinal $\lambda$ of uncountable cofinality it is consistent that there is a countably tight Boolean algebra $A$ with a distinguished ultrafilter $\infty$ such that for every $a \not \nexists \infty$ the algebra $A \mid a$ is countable and $\infty$ has hereditary character $\lambda$.
\end{abstract}

\section{INTRODUCTION}

In this paper, we use various versions of the following forcing notion:

$$
P(A, u)=\left\{\left(p_{-1}, p_{1}\right) \in[A]^{2}:\left(p_{-1} \cup p_{1}\right) \notin u, p_{-1} \cap p_{1}=\emptyset\right\},
$$

with the order defined by $p \leq q$ if and only if $p_{i} \supseteq q_{i}$ for $i=1,-1$, where $A$ is a subalgebra of the algebra $\wp(\omega)$ and $u$ is an ultrafilter. Similar notions of forcing were used in [G], [BK], [Sh1], [Sh2]. We are using combinatorial notation for

Received by the editors June 6, 1994 and, in revised form, January 20, 1997.

1991 Mathematics Subject Classification. Primary 03E35, 03E50, 03E99, 06E15, 06E99, 54A25, 54A35, 54B35, 54G12, 54G99, 54H10.

Some results presented in this paper were obtained when the author was a Ph.D. student at the University of Toronto, under the supervision of Professors F.D. Tall and W. Weiss. Other results of this paper were obtained when the author was a National Science and Engineering Research Council of Canada postdoctoral fellow at York University, under the supervision of Professor J. Steprans. 
Boolean algebraic operations i.e., $\cup, \cap,-, \emptyset$, that is we treat Boolean algebras, most of the time, as fields of sets, in most cases fields of subsets on the set of integers. The complement of $a$ will be denoted by $a^{-1}$. It can be seen that if $G \subseteq P(A, u)$ is a generic filter in $P(A, u)$ meeting sufficiently many dense sets (see $[\mathrm{K}]$ for definitions), then $g(A, u)=\bigcup\left\{p_{1}: \exists p_{-1}\left(p_{-1}, p_{1}\right) \in G\right\}$ has the property that only one ultrafilter (namely $u$ ) does not generate an ultrafilter in the algebra $\langle A \cup\{g(A, u)\}\rangle$ generated by $A$ and $g(A, u)$. Thus, the extension $\langle A \cup\{g(A, u)\}\rangle$ of the algebra $A$ obtained by adding the element $g(A, u)$ is an example of a minimal extension, the notion introduced and investigated by S. Koppelberg in [Kop1] and [Kop2], and present implicitly in [BK], [Sh1], [Sh2]. Following S. Koppelberg, by a minimally generated Boolean algebra we mean an algebra $A$ generated by the family $\left\{a_{\xi}: \xi<\eta\right\}$ where for every $\xi<\eta$ the element $a_{\xi}$ is minimal over $\left\langle\left\{a_{\xi^{\prime}}: \xi^{\prime}<\xi\right\}\right\rangle_{A}$. In this paper we investigate various ways of constructing minimally generated Boolean algebras (compact spaces) using versions of forcings $P(A, u)$.

In section 2, we recall elementary properties of minimally generated algebras and we propose some specific ways of viewing some minimally generated algebras which correspond to Fedorchuk's language of spectras. This establishes a link between the Boolean algebras we deal with and some trees.

In section 3, we we deal with versions of the forcing notion $P(A, u)$.

In the remaining sections we provide several constructions that serve as examples or counterexamples in the theory of cardinal functions on Boolean algebras or compact spaces (see surveys [M1], [Ju1], [Ju2], [Ju3], [H]). The general problem from this theory which we consider is :

what are the possible gaps between versions of independence: tightness, spread, hereditarily density; and versions of character: hereditary character, minimal character, hereditary minimal character (sometimes called pseudoaltitude (see [vD])), hereditary weight.

In particular, we are interested in the gaps between the countable versions of independence and $h \chi, h \min \chi, \min \chi$, or $h w$. In order to discuss our constructions we need to recall (following the approach of $[\mathrm{vD}]$ ) a few definitions of cardinal functions on Boolean algebras or compact 0-dimensional spaces. Let $A$ denote a Boolean algebra and $X$ its Stone space; any point $x \in X$ is considered as an ultrafilter of $A$. In this context $a \in x$ means the same as $x \in a$, for $a \in A$ and $x \in X$ (for introduction to Stone's duality see [Si]). Consider the following Boolean equation:

$$
\bigcap_{\xi \in F} a_{\xi} \cap \bigcap_{\xi \in G} a_{\xi}^{-1} \neq \emptyset .
$$

A family $\left\{a_{\xi}: \xi<\kappa\right\} \subseteq A$ is called an independent family if and only if $\left(^{*}\right.$ ) holds for every disjoint $F, G \in[\kappa]^{<\omega}$. It is called a discrete family if and only if $(*)$ holds for every disjoint $F, G \in[\kappa]^{<\omega}$, where $|F|=1$. A sequence $\left\{a_{\xi}: \xi<\kappa\right\}$ is called a free sequence if and only if $\left(^{*}\right)$ holds for every $F, G \in[\kappa]^{<\omega}$, such that $\max F<\min G$. Finally a sequence is called a left-separated sequence if and only if $(*)$ holds for every $F, G \in[\kappa]^{<\omega}$, such that $\max F<\min G$ and $|F|=1$.

Now, we may define (following the approach from [vD]) various versions of independence. The independence of a Boolean algebra $A$ (abbreviated $\operatorname{ind}(A)$ in the sequel) is the supremum of all $\kappa$ such that there is an independent family of elements of $A$ of size $\kappa$. The spread of a Boolean algebra $A$ (abbreviated $s(A)$ in the 
sequel) is a supremum of all $\kappa$ such that there is a discrete family of elements of $A$ of size $\kappa$. The tightness of a Boolean algebra $A$ (abbreviated $t(A)$ in the sequel) is a supremum of all $\kappa$ such that there is a free sequence of elements of $A$ of length $\kappa$. The hereditary density of a Boolean algebra $A$ (abbreviated $h d(A)$ in the sequel) is a supremum of all $\kappa$ such that there is a left-separated sequence of elements of $A$ of length $\kappa$. We will say that $A$ is hereditarily separable if its hereditary density is countable. Note that $i n d \leq t \leq s \leq h d$ (see [vD]). For topological translations see $[\mathrm{H}]$ or $[\mathrm{vD}]$.

Now, let us recall the other group of definitions of character, hereditary character, minimal character of a point and hereditary minimal character (also called pseudoaltitude) and hereditary weight:

character: $\quad \chi(x, A)=\chi(x, X)=\min \{|\mathcal{U}|: \mathcal{U} \subset x: \mathcal{U}$ generates $x\}$;

hereditary character:

$$
\begin{aligned}
h \chi(x, X) & =\min \{\chi(x, Y): Y \subset X, \chi(x, Y) \geq \omega, Y \text { is closed }\} \\
& =\min \{\chi(x / J, A / J): J \cap x=\emptyset, J \text { is an ideal of } A, x / J \text { is nonprincipal }\}
\end{aligned}
$$

minimal character:

$$
\min \chi(X)=\min \{\chi(x, X): x \in X\}
$$

character:

$$
\chi(X)=\sup \{\chi(x, X): x \in X\}
$$

hereditary minimal character: $h \min \chi(X)=p a(A)=\min \{h \chi(x, X): x \in X\}$;

hereditary weight: $h w=\min \{|B|: B$ is an infinite homomorphic image of $A\}$.

Note that $h \min \chi=p a \leq \min \chi, h \chi \leq \chi ; h \chi, h \min \chi \leq h w \leq 2^{\omega}$.

The class of minimally generated algebras seems to be a suitable class of Boolean algebras for obtaining the gaps between the above two groups of cardinal functions. By a result of $\mathrm{S}$. Koppelberg, $\operatorname{ind}(A)$ is countable for any minimally generated algebra (see [Kop1]). Our result from section 3 says that if a forcing $P(A, u)$ satisfies c.c.c., then $A$ has countable tightness, a fact that enables us to deduce countable tightness from this forcing theoretic property of $P(A, u)$. (A surprising application of minimally generated algebras to fixed point theory in linear spaces is presented in [Kosz2].)

In section 4, we construct a generic version of a classical example (in its topological version due to Fedorchuk [Fe1]), of a hereditarily separable Boolean space with $h w$ uncountable (thus, without a convergent sequence). The original construction was obtained under the assumption of $\diamond$. Our construction can also be carried out under this assumption, in the style of the construction from [BK]. The point of our forcing approach is to generalize Fedorchuk's construction to higher cardinals. We prove that for every cardinal $\lambda$ of uncountable cofinality it is consistent that there is a Boolean algebra $A_{\lambda}$ which is hereditarily separable (i.e., $h d\left(A_{\lambda}\right)=\omega$ ) and all of whose infinite homomorphic images have cardinality $\lambda$. Thus, we obtain a gap of the type $(\omega, \lambda)$ between $h d$ and $h w$ for any $\lambda$ of uncountable cofinality.

The algebra $A_{\lambda}$ can be also used to prove that problem 39 from [M1] (which is problem 35 from [M2]) has an independent answer. The problem is whether $h \chi=\left(\chi_{H-}=\operatorname{Card}_{H_{-}}\right)=h w$, i.e., whether the minimal infinite cardinal which is the character of a homomorphic image of an algebra $A$ is the same as the minimal 
size of an infinite homomorphic image of $A$. It is consistent to have $h \chi(A)=\omega_{1}$ while $h w(A)=\omega_{2}=2^{\omega}$. The algebra $A_{\omega_{2}}$ of this section works. It has all points of hereditary character $\omega_{1}$, but all its homomorphic images have size $\omega_{2}$. In fact $\omega_{2}$ can be replaced here by any cardinal of uncountable cofinality (thus, making the gap between $h \chi$ and $h w$ arbitrarily large). On the other hand $\mathrm{CH}$ implies the equality $h \chi=h w \leq \omega_{1}$ because, otherwise, we get a convergent sequence to a first countable point in some closed subspace and this sequence gives rise to a second countable closed subspace.

Let us comment on the gaps between hereditary minimal character and the versions of independence. Hereditary minimal character of ours or Fedorchuk's hereditarily separable Boolean algebras is $\omega_{1}$ and it is an open problem (see [vDMR]) whether hereditary minimal character is bounded by $\omega_{1}$ in general for all Boolean algebras. It is true under $\mathrm{CH}$ and in many models of $\neg \mathrm{CH}$ (see [Kosz1] or [Just] for a related result). It was proved in [BSV] that $\operatorname{ind}(A)>\omega$ implies $h \min \chi(A) \leq \omega_{1}$ and later in [JSz] that $t(A)>\omega$ implies $h \min \chi(A) \leq \omega_{1}$. Moreover for countably tight spaces PFA implies that $h \min \chi$ is countable (by a result from [Bal] which says that PFA implies that every compact, countably tight space is sequential) and for hereditary separable compact spaces $\mathrm{MA}+\neg \mathrm{CH}$ itself implies that $\chi \geq h \min \chi$ is countable (by a result from $[\mathrm{Sz}]$ which says that $\mathrm{MA}+\neg \mathrm{CH}$ implies that there are no compact S-spaces ). For surveys of similar problems see [N2], [vDMR].

In section 5, we construct a certain modification of forcing $P(A, u)$ and we use it for proving that for every cardinal $\lambda$ of uncountable cofinality it is consistent that the size of the continuum is $\lambda$ and there is the hereditarily separable Boolean algebra all of whose infinite homomorphic images are of size $\omega_{1}$. We also note that the above algebra may exist in the same model with a hereditarily separable algebra all of whose infinite homomorphic images have size $\lambda$, constructed in section 4 .

In section 6, we derive certain algebras from the constructions obtained in sections 4 and 5 . To explain their properties we need some definitions.

$$
c_{H r}(A)=\{(\kappa, \lambda): \text { There is an infinite homomorphic image } B \text { of } A \text {, }
$$

$$
|B|=\lambda, c(B)=\kappa\}
$$

where $c(B)$ denotes the cellularity of $B$, that is the supremum over sizes of pairwise disjoint subsets of $B$. (Note that $\sup \{c(B): B$ is an infinite homomorphic image of $A\}=s(A)$, see $[\mathrm{vD}]$.)

Using the constructions from sections 4 and 5 we obtain a model of ZFC and $2^{\omega}=\omega_{2}$ in which there are Boolean algebras $A, B, C, D$ such that

$c_{H r}(A)=\left\{\left(\omega, \omega_{2}\right)\right\}$,

$c_{H r}(B)=\left\{\left(\omega, \omega_{1}\right)\right\}$,

$c_{H r}(C)=\left\{\left(\omega, \omega_{2}\right),\left(\omega_{1}, \omega_{2}\right)\right\}$,

$c_{H r}(D)=\left\{\left(\omega, \omega_{1}\right),\left(\omega_{1}, \omega_{1}\right)\right\}$.

In fact $\omega_{2}$ can be replaced above by any cardinal $\lambda$ of uncountable cofinality.

Using these algebras we answer several questions of D.Monk from [M2] which ask whether a given set of pairs of cardinals can consistently be the set $c_{H r}(E)$ for some Boolean algebra $E$.

The algebra $A$ gives the positive answer to Problem 8 i). The algebra $C$ gives the positive answer to problem 8 ii). Clopen $(U l t(A) \cup U l t(B))$ gives the positive answer to Problem $8 \mathrm{v})$ by providing an algebra whose $c_{H r}$ is $\left\{\left(\omega, \omega_{1}\right),\left(\omega, \omega_{2}\right)\right\}$. Problem 
$8 \mathrm{vi})$ is solved in the positive by noting that $c_{H r}$ of the algebra $\operatorname{Clopen}(\operatorname{Ult}(B) \cup$ $\operatorname{Ult}(C))$ is $\left\{\left(\omega, \omega_{1}\right),\left(\omega, \omega_{2}\right),\left(\omega_{1}, \omega_{2}\right)\right\}$. Problem 8 vii) is solved in the positive by noting that the $c_{H r}$ of the algebra Clopen $(U l t(B) \cup \beta \omega)$ is $\left\{\left(\omega, \omega_{1}\right),\left(\omega, \omega_{2}\right),\left(\omega_{1}, \omega_{2}\right)\right.$, $\left.\left(\omega_{2}, \omega_{2}\right)\right\}$ (note that $|\wp(\omega)|=\omega_{2}$ in our model). Problem 8 viii) is solved in the positive by observing that the $c_{H r}$ of the algebra Clopen $(\operatorname{Ult}(C) \cup \operatorname{Ult}(D))$ is $\left\{\left(\omega, \omega_{1}\right),\left(\omega_{1}, \omega_{1}\right),\left(\omega_{2}, \omega_{2}\right)\right\}$. Problem $\left.8 \mathrm{xvi}\right)$ is solved in the positive by making an observation that the $c_{H r}$ of the algebra Clopen $(U l t(C) \cup$ convergent sequence) is $\left\{(\omega, \omega),\left(\left(\omega, \omega_{2}\right),\left(\omega_{1}, \omega_{2}\right)\right\}\right.$. Problem $\left.8 \mathrm{xx}\right)$ is solved in the positive by making an observation that the $c_{H r}$ of the algebra Clopen $\left(U l t(C) \cup \operatorname{Ult}\left(\right.\right.$ FinCofin $\left.\left.\left(\omega_{1}\right)\right)\right)$ is $\left\{(\omega, \omega),\left(\omega_{1}, \omega_{1}\right),\left(\omega, \omega_{2}\right),\left(\omega_{1}, \omega_{2}\right)\right\}$. Problem 8 xxii) is solved in the positive by noting that the $c_{H r}$ of the algebra Clopen $(U l t(C) \cup U l t(B) \cup$ convergent sequence) is $\left\{(\omega, \omega),\left(\omega, \omega_{1}\right),\left(\omega, \omega_{2}\right),\left(\omega_{1}, \omega_{2}\right)\right\}$.

In section 7 , we construct a model of $\mathrm{MA}+\neg \mathrm{CH}$ in which there is a minimally generated Boolean algebra $A$ with its Stone space $X$ such that

4) $\min \chi(x)=\omega_{1}$

5) $t(A)=t(X)=\omega$.

Let us make comments on the gaps between $\min \chi$ and the versions of independence. Interval algebras give ZFC examples of countably independent Boolean spaces (see [Kop3]) with arbitrarily large minimal character. Thus the gap between ind $=\omega$ and $\min \chi$ is arbitrarily large in ZFC. It was proved in [Kosz1] that in many models of $\neg C H, \operatorname{ind}(A)=\omega$ implies that $\min \chi(A) \leq \omega_{1}$ for $A \subseteq \wp(\omega)$ and later this method was applied in [Ju4] for concluding that $t(A)=\omega$ implies $\min \chi(A) \leq \omega_{1}$ in the same class of models. On the other hand, it is still an open question (due to Juhasz) whether it is true in ZFC that countably tight Boolean spaces have a point of character $\leq \omega_{1}$. CH implies that $\omega_{1}$ is attained as the minimal character in countably tight spaces (by a result of V.Malykhin [Mal]). PFA implies that it is not the case i.e., there is always a point of countable character (by a result of A.Dow [Do]). V. Malykhin has also proved (see $[\mathrm{Mal}])$ that $\mathrm{MA}(\sigma$-centered $)+\neg \mathrm{CH}$ (see $[\mathrm{W}]$ ) is consistent with the existence of a countably tight Boolean algebra without a point of countable character. The model considered by Malykhin is a generic extension of any model by one Cohen real; thus these models do not satisfy MA. I. Juhasz has found a principle implicit in Malykhin's proof which he calls $(t)$ (that is a weakening of the principle $\boldsymbol{\alpha}$, thus also a weakening of $\diamond$ ) under which he constructed countably tight compact spaces with $\min \chi$ equal to $\omega_{1}$ (see [Ju5]). MA $+\neg \mathrm{CH}$ for partial orders having precaliber $\omega_{1}$ implies the failure of $(t)$ (for proof see section 7). Thus, our example from section 7 , in the presence of full $\mathrm{MA}+\neg \mathrm{CH}$, provides new models where there exist countably tight, compact spaces of uncountable minimal character.

We also prove that the algebra obtained in section 7 , although without an ultrafilter which is countably generated, is a subalgebra of an algebra all of whose ultrafilters have countable character. This provides an example of a first countable compact space (the Stone space of the bigger algebra) which has a continuous image without a point of countable character, answering a question of A.V. Arhangel'skii (see [Shah], question 4.6., the construction also answers question 4.4. ii). This result shows that the search for properties of compact spaces which would guarantee in ZFC the existence of a point of countable character (see [Shah]) must be limited to properties which are not preserved by continuous mappings of compact spaces.

In section 8, we consider yet another way of viewing some minimally generated Boolean algebras. 
In section 9, using the approach from section 8 and assuming the existence of a family $\mathcal{F} \subseteq[\lambda]^{\omega}$ with some combinatorial properties whose existence is consistent for any cardinal $\lambda$ of uncountable cofinality, we construct a forcing that adds another example of a minimally generated Boolean algebra.

The minimally generated Boolean algebra $A$ (its Stone space $X$ ) that is obtained in section 9 has a distinguished ultrafilter (point) $\infty$ which has the following properties.

1) $h \chi(\infty)=\lambda$.

2) $\forall a \in A a \notin \infty(\infty \notin a) \Rightarrow|A| a \mid=\omega$.

3) $t(A)=t(X)=\omega$.

For regular $\lambda$, the consistent existence of the above algebra can be easily concluded from results of [N1]. Our method works also for singular $\lambda$ of uncountable cofinality. Hence, it is possible to obtain an arbitrary large gap between countable tightness and $h \chi$ (obviously $h \chi$ is always less or equal to the continuum). Note that the results from [Bal] and $[\mathrm{Sz}]$ show that the existence of the space as above cannot be proved in ZFC.

Those constructions characterize possible values of hereditary character in compact spaces of countable tightness. By the above results those values can be any cardinals of uncountable cofinality. It is also easy to see (see section 9) that a cardinal of countable cofinality can be a hereditary character of a point in a compact space if and only if it is $\omega$. A sharper result for $\lambda=\omega_{2}$ has been recently obtained by M. Rabus, who consistently constructed a countably tight algebra with a distinguished point to which there is no $\omega$ - or $\omega_{1}$-convergent sequence (see [Ra]).

As can be seen from the preceding text, we use the Boolean algebraic as well as topological language, switching when convenient. This should not cause problems since it requires only basic knowledge of the Stone duality. As a reference concerning elementary theory of Boolean algebras we suggest the first chapter of the book [Si].

The notation used in this paper is inconsistent in a fairly standard way; it is mostly based on the notation from $[\mathrm{K}]$ and $[\mathrm{KV}]$. Here is a short list of some less standard symbols. If $A$ is a Boolean algebra, then $U l t(A)$ denotes the Stone space of $A ; C \operatorname{Clopen}(X)$ denotes the Boolean algebra of clopen subsets of space $X ; A t(A)$ denotes the ideal generated by the atoms of $A$. $A \mid a$ for $a \in A$ denotes the algebra generated by elements of $A$ below $a$; the unit of $A \mid a$ is identified with $a$. If $X \subseteq A$, then by $\langle X\rangle_{A},\langle X\rangle_{A}^{I d},\langle X\rangle_{A}^{F i}$ we denote, respectively, the subalgebra, the ideal and the filter generated by $X$ in $A$; we will often omit the subscripts. $X^{+}$denotes the set of all complements of elements of $X . A\langle x\rangle$ denotes the one-extension of $A$ obtained by adding an element $x$. If $J$ is an ideal of $A$, then by $x / J, X / J, A / J$ we mean the image of, respectively, $x, X, A$ under the canonical homomorphism from $A$ into the quotient algebra $A / J$. We use combinatorial terminology with respect to Boolean algebraic operations; this includes such expressions as subset, superset, intersection, etc, as well as their symbols $\subseteq, \supseteq, \cap$. Sometimes we use notation $a^{-1}$ for the complement of $a, a^{1}$ for $a$ and $a^{0}$ for the unit of a Boolean algebra. $\{0,1\}$ may denote the two element Boolean algebra.

$f^{\prime \prime}(X)$ denotes the image of $X$ under a function $f . f \mid X$ denotes the restriction of $f$ to $X$. $\subset, \supset$ refer to strict inclusion unlike $\subseteq, \supseteq$, which include the possibility of equality. If $\mathcal{F}$ is a family of sets, then $\mathcal{F} \mid X=\{Y \in \mathcal{F}: Y \subset X\}$. The notation referring to iterated forcing is based on the paper [Ba1]. If the forcing sign $\|-$ is used in the text, the sentence, in the forcing language, to which it applies is italicized. 


\section{Minimal extensions}

Reading this section requires from the reader basic knowledge of Boolean algebras which can be found e.g. in [Si]. For example we will be using the fact that if $A$ is a proper subalgebra of $B$, then there is an ultrafilter of $A$ which does not generate an ultrafilter in $B$ (i.e., that compact topologies are minimal among Hausdorff topologies), or that if generators of a Boolean algebra generate a filter, then it is an ultrafilter, or that any element of a one-extension $A\langle x\rangle$ of $A$ is of the form $a_{1} \cap x \cup a_{2} \cap x^{-1}$ for some $a_{1}, a_{2} \in A$.

Definition 2.1. Let $Z$ be a set. Suppose that $A \subseteq \wp(Z)$ is a Boolean algebra and $u$ is an ultrafilter of $A$. An element $x \subseteq Z$ is called minimal for $(A, u)$ if and only if $u$ is the only ultrafilter in $A$ which does not generate an ultrafilter in the algebra $A\langle x\rangle$.

Our terminology is motivated by paper [Kop1], where an extension $B$ of a Boolean algebra $A$ is called minimal if and only if there is no $C$ such that $A \subset$ $C \subset B$. It is proved in [Kop1] that $B$ is a minimal extension of $A$ if and only if $B=A\langle x\rangle$ for some (any) $x \in B-A$ and $x$ is minimal for $(A, u)$ for some ultrafilter $u$ of $A$. Most of the observations of this section are reformulations of results obtained by S. Koppelberg. Minimal elements were implicitly considered by S. Shelah ([Sh1], [Sh2]), S. Grigorieff ([G]) and J. Baumgartner and P. Komjath ([BK]). The following is a reformulation of a result obtained in [Kop1].

Proposition 2.2. Suppose that $A \subseteq \wp(Z)$ is a Boolean algebra, $u$ is an ultrafilter of $A, x \subseteq Z$ and $x \notin A$; then the following are equivalent:

a) $\{a \in A: a \cap x \in A\}=u^{+}$,

b) $\{a \in A: a \cap x \notin A\}=u$,

c) $x$ is minimal for $(A, u)$.

Proof. a) $\rightarrow$ b). If $a \cap x \notin A$, then by a) we have $a \notin u^{+}$, so $a \in u$ as $u$ is an ultrafilter. If $a \in u$, then $a^{-1} \notin u$ and by a) $x \cap a^{-1} \in A$, so since $x \notin A$ and $x=x \cap a^{-1} \cup x \cap a$ we have $x \cap a \notin A$.

b) $\rightarrow$ c). As $x \notin A$, some ultrafilter of $A$ does not generate an ultrafilter in $A\langle x\rangle$, so it is enough to prove that, for each ultrafilter $v \neq u$ of $A, v$ generates an ultrafilter in $A\langle x\rangle$.

Let $c \in v-u$; then $c \cap x \in A$ and so $c \cap x^{-1}=c-(c \cap x) \in A$. Now let $v^{\prime}$ be an ultrafilter of $A\langle x\rangle$ such that $v \subseteq v^{\prime}$, and take $y \in v^{\prime} ; y$ is of the form $b_{1} \cap x \cup b_{2} \cap x^{-1}$ for some $b_{1}, b_{2} \in A$, so $y \cap c=b_{1} \cap x \cap c \cup b_{2} \cap x^{-1} \cap c \in A$, so $y \cap c \in v^{\prime} \cap A=v$ and $y \cap c \subseteq y$, so $v$ generates $v^{\prime}$.

c) $\rightarrow$ a). Suppose $a \cap x \in A$; then $a \cap x^{-1}=a-(a \cap x) \in A$ and so for every $y \in A\langle x\rangle$ we have $a \cap y \in A$ (as $y=b_{1} \cap x \cup b_{2} \cap x^{-1}$ for some $b_{1}, b_{2} \in A$ ) so every ultrafilter $v$ of $A$ containing $a$ generates an ultrafilter in $A\langle x\rangle$, hence $a \notin u$, so $a \in u^{+}$.

If $a \in u^{+}$, then by the minimality of $x$ for $(A, u)$, every ultrafilter $v$ of $A$ containing $a$ generates an ultrafilter of $A\langle x\rangle$, hence $a \cap x$ is in the algebra $A \mid a$, so $a \cap x \in A$.

Lemma 2.3. Let $A \subseteq \wp(Z)$ be a Boolean algebra and let $u$ be its ultrafilter. Then $x$ is minimal for $(A, u)$ if and only if $x^{-1}$ is minimal for $(A, u)$.

Proof. Suppose $x$ is minimal for $(A, u)$; then by proposition 2.2 we have that $\{a \in$ $A: a \cap x \notin A\}=u$. Now, if $a \in u$, we have $a \cap x^{-1}=a-(a \cap x)$, so $a \cap x^{-1} \notin A$. Also if 
$a \cap x^{-1} \notin A$, then $a \cap x=a-\left(a \cap x^{-1}\right) \notin A$, so $a \in u$ and so $\left\{a \in A: a \cap x^{-1} \notin A\right\}=u$; hence by proposition 2.2 the element $x^{-1}$ is minimal for $(A, u)$. The opposite implication is symmetric.

Lemma 2.4. Suppose that $A \subseteq B \subseteq \wp(\omega)$ are Boolean algebras, and $u$ is an ultrafilter of $A$ which generates an ultrafilter $v$ of $B$. If $x$ is minimal for $(A, u)$, then $x$ is minimal for $(B, v)$.

Proof. If $b \in v^{+}$, then there is $a \in u^{+}$such that $b \subseteq a$. Then $b \cap x=b \cap a \cap x$. But $a \cap x \in A$ by proposition 2.2 , so $b \cap x \in B$. Thus every ultrafilter of $B$ containing $b$ generates an ultrafilter in $B\langle x\rangle$, so $v$ is the only ultrafilter of $B$ which does not generate an ultrafilter in $B\langle x\rangle$; hence $x$ is minimal for $(B, v)$.

Definition 2.5 ([Kop1]). A Boolean algebra $A$ is called a minimally generated algebra if and only if $A=\left\langle\left\{a_{\alpha}: \alpha<\beta\right\}\right\rangle$ for some ordinal $\beta$ and for every $\alpha<\beta$ the element $a_{\alpha}$ is minimal for $\left(A_{\alpha}, u_{\alpha}\right)$, where $A_{\alpha}=\left\langle\left\{a_{\gamma}: \gamma<\alpha\right\}\right\rangle$ and $u_{\alpha}$ is some ultrafilter of $A_{\alpha}$.

Now we present a certain way of building minimally generated algebras, where a set of distinct generators is indexed by nodes of a tree. Algebras built this way have the peculiar property that ultrafilters correspond exactly to maximal branches of a tree which indexes irredundant generators of the algebra, along which we carry out the construction of the algebra. This property will be essential in many constructions we provide in the following sections of the paper. This property easily enables us to identify ultrafilters but also plays an important role when, as in section 7 , we force with forcings that do not add any uncountable branches through $\omega_{1}$-trees.

Definition 2.6. A subtree $T$ of $S=\left\{f: \exists \alpha<\omega_{1} f: \alpha+1 \rightarrow\{-1,1\}\right\}$ with inclusion as the order is called acceptable if and only if $T$ is downward closed in $S$ and $s \frown i \in T$ implies $s \frown(-i) \in T$ for any $s \in 2^{<\omega_{1}}$ and $i \in\{-1,1\}$. We say $T_{1} \prec T_{2}$ if and only if $T_{2}$ end-extends $T_{1}$, for acceptable $T_{1}, T_{2}$.

Definition 2.7. Let $T$ be an acceptable tree. Then a Boolean algebra $A$ is called a $T$-algebra if and only if $A$ is generated by a set of distinct generators $\left\{a_{t}: t \in T\right\}$ and

1) For every $t \in T$ the filter generated by $\left\{a_{s}: s<t\right\}$ denoted by $u_{t}$ is a proper filter and $a_{t}$ is minimal for $\left(A_{t}, u_{t}\right)$, where $A_{t}$ denotes the algebra generated by the elements $\left\{a_{s}: s<t\right\}$.

2) For every $t \in 2^{<\omega_{1}}$ such that $t \frown i \in T$ for some $i \in\{-1,1\}$, we have

$$
a_{t \frown i}=\left(a_{t \frown(-i)}\right)^{-1} \text {. }
$$

If $T_{1} \prec T_{2}$ are acceptable trees and $A_{i}$ is a $T_{i}$ algebra for $i=1,2$, then we say that $A_{1} \prec A_{2}$ if and only if there is an embedding of Boolean algebras $i: A_{1} \rightarrow A_{2}$, such that $i\left(a_{t}\right)=b_{t}$, where $A_{1}=\left\langle\left\{a_{t}: t \in T_{1}\right\}\right\rangle, A_{2}=\left\langle\left\{b_{t}: t \in T_{2}\right\}\right\rangle$.

Lemma 2.8. Let $A$ be a T-algebra for some acceptable tree $T$. Let $u$ be an ultrafilter of $A$. Then there is a maximal branch $b$ of $T$ such that

$$
u=u_{b}=\left\langle\left\{a_{t}: t \in b\right\}\right\rangle_{A}^{F i} .
$$

Moreover any filter of the form $u_{b}$ for a maximal branch $b$ of $T$ is an ultrafilter in A. 
Proof. Assume that $u$ is an ultrafilter. First note that there is some maximal branch $b$ of $T$ such that $u_{b} \subseteq u$. Define $b$ by induction. If we have defined $b \mid \xi$ for $\xi<h t(T)$ such that $u_{b \mid \xi} \subseteq u$ and $b \mid \xi$ is not a maximal branch, then $(b \mid \xi) \frown(-1),(b \mid \xi) \frown 1 \in T$, because $T$ is acceptable. Since $u$ is an ultrafilter of $A$, we conclude that either $a_{b \mid \xi-(-1)} \in u$ or $a_{b \mid \xi \frown 1} \in u$, (by 2 of definition 2.7) so we can extend $b \mid \xi$ to $b \mid(\xi+1)$. Now, in order to finish the proof of the lemma, it is enough to see that $u_{b}$ is an ultrafilter of $A$ for every maximal branch $b$ of $T$.

We prove by induction on the height of an acceptable tree $T$ that for every maximal branch $b$ of $T$ the filter $u_{b}$ is an ultrafilter. If the height is a limit ordinal, the proof is immediate by the inductive assumption, as $u_{b \mid a}$ 's are ultrafilters in the algebras corresponding to trees $T \mid \alpha$ for $\alpha<h t(T)$. If the height is a successor, say $\beta+1$, then the inductive assumption implies that $u_{b \mid \beta+1}$ is an ultrafilter in $T \mid \beta+1$. It stays an ultrafilter in the algebra $B$ generated by $\left\{a_{t}: t \in T, b(\beta) \nsupseteq t\right\}$ because all new elements in $B$ do not extend $u_{b \mid \beta+1}$ (by 2.7. 1)). Now $u_{b}$ generates an ultrafilter in $B\left\langle a_{b(\beta+1)}\right\rangle$, because the latter algebra is generated over $B$ by an element in $u_{b}$.

Example 2.9. The free algebra with countably many free generators, i.e., the algebra of clopen subsets of the Cantor set, $2^{\omega}$ is a $\left(2^{<\omega}-\{\emptyset\}\right)$-algebra.

For convenience we work with $2^{\{-1,1\}}$ instead of $2^{\omega}$. For $s \in 2^{\{-1,1\}}$ put

$$
a_{s \frown 1}=\left\{x \in\{-1,1\}^{\omega}: s \frown 1 \subseteq x\right\}, a_{s \frown-1}=\left(a_{s \frown 1}\right)^{-1} .
$$

Take $a_{s \frown 1}$ and consider $t \subseteq s$. If $t=r \frown 1$, then $a_{s \frown 1} \subseteq a_{t}$. If $t=r \frown(-1)$, $a_{r \frown 1} \cap a_{s \frown 1}=\emptyset$, so since $a_{t}=a_{r \frown(-1)}=\left(a_{r}-1\right)^{-1}$ we get $a_{s \frown 1} \subseteq a_{t}$ as well.

So the family $\left\{a_{t}: t \subseteq s\right\}$ generating $u_{s} \frown 1$ has the finite intersection property; hence it generates an ultrafilter in $A_{s \frown 1}$. We have to show that $a_{s \frown 1}, a_{s \frown(-1)}$ are minimal for $\left(A_{s \frown 1}, u_{s \frown 1}\right)=\left(A_{s \frown(-1)}, u_{s \frown(-1)}\right)$. By lemma 2.3 , it is enough to show that $a_{s \frown 1}$ is minimal for $\left(A_{s \frown 1}, u_{s \frown 1}\right)$. By proposition 2.2, since $\left(a_{t}: t \subseteq s\right)$ generates $A_{s \frown 1}$, it is enough to show that $a_{t}^{-1} \cap a_{s \frown 1} \in A_{s \frown 1}$ for $t \subseteq s$. We have seen above that $a_{s \frown 1} \subseteq a_{t}$ for $t \subseteq s$, so it follows that $a_{t}^{-1} \cap a_{s \frown 1}=\emptyset \in A_{s \frown 1}$, for $t \subseteq s$.

Now let us note several elementary facts regarding $T$-algebras for an acceptable tree $T$.

Fact 2.10. Suppose $A$ is a $T$-algebra for some acceptable tree $T$. Then $A$ is a minimally generated algebra.

Proof. Order the generators of $A$ " $T$-level-wise" and see that this ordering satisfies definition 2.5.

Fact 2.11. Suppose that $A$ is a $T$-algebra for some acceptable tree $T$. Suppose that cofinality of every maximal branch of $T$ is greater or equal to a cardinal $\kappa$. Then $\min \chi(A) \geq \kappa$.

Fact 2.12. Suppose that $T$ is a $T$-algebra for some acceptable tree $T$. Suppose that cofinality of some maximal branch of $T$ is greater or equal to a cardinal $\kappa$. Then $\chi(A) \geq \kappa$. 


\section{Fording minimal eXtensions}

In this section, first we present a way in which minimal elements for a pair $(A, u)$ can be obtained using a forcing notion $P(A, u)$; then we prove some properties of the forcing $P(A, u)$ and of some related forcings.

Definition 3.1. Let $A \subseteq \wp(\omega)$ be a Boolean algebra, and let $F$ be a filter in $A$; then

$$
\begin{aligned}
P(A, F)= & \left\{p: p=\left(p_{-1}, p_{1}\right) \in A^{2}, p_{-1} \cap p_{1}=\emptyset, p_{-1} \cup p_{1} \notin F\right\}, \\
& \left(p_{-1}, p_{1}\right) \leq\left(q_{-1}, q_{1}\right) \text { iff } p_{-1} \supseteq q_{-1}, p_{1} \supseteq q_{1} .
\end{aligned}
$$

An element $g \subseteq \omega$ is called $(A, F)$-minimal over $\mathcal{M}$ if and only if there is a generic filter $G \subseteq P(A, F)$ which meets all the dense subsets of $P(A, F)$ from $\mathcal{M}$ such that $g=\bigcup\left\{p_{1}: \exists\left(p_{1}, p_{-1}\right) \in G\right\}$.

Fact 3.2. Let $A \subseteq \wp(\omega)$ be a Boolean algebra and $u$ a nonprincipal ultrafilter of $A$. If $\mathcal{M}$ is a model of sufficiently large fragment of set theory, $\mathcal{M} \cap A$ generates $u$ and $A, u \in \mathcal{M}$, then every $(A, u)$-minimal element over $\mathcal{M}$ is minimal for the pair $(A, u)$.

Proof. For $a \in u^{+} \cap \mathcal{M}$, define

$$
D_{a}=\left\{p \in P(A, u): a \subseteq p_{-1} \cup p_{1},\left(p_{-1}-a\right) \neq \emptyset,\left(p_{1}-a\right) \neq \emptyset\right\} .
$$

Nonprincipality of $u$ implies that the sets $D_{a}$ are dense. We may assume that each $D_{a} \in \mathcal{M}$. Let $g \subseteq \omega$ be $(A, u)$-minimal over $\mathcal{M}$. Find $p \in G$ such that $p \in D_{a}$. Then $p_{1} \subseteq g, p_{-1} \subseteq g^{-1}$, so $g \cap a=p_{1} \cap a$; hence, for every $a \in u^{+} \cap \mathcal{M}$ we have $a \cap g \in A$, i.e., $u^{+} \cap \mathcal{M} \subseteq\{a \in A: a \cap g \in A\}$ and $u^{+} \cap \mathcal{M}$ generates a maximal ideal of $A$. Also $g \neq a, a^{-1}$ for $a \in u^{+}$, so $\{a \in A: a \cap g \in A\}$ is a proper ideal. Hence, $u^{+}=\{a \in A: a \cap g \in A\}$, and so $g$ is minimal for $(A, u)$ by Proposition 2.2 .

Fact 3.3. Let $A$ be a Boolean algebra, $u$ an ultrafilter in $A$. If $P(A, u)$ satisfies the c.c.c., then the following conditions are satisfied:

$$
\forall a \in u^{+}|A| a \mid \leq \omega
$$

$$
t(A)=\omega .
$$

Proof. If $\exists a \in u^{+}|A| a \mid>\omega$, then $\{(b, a-b): b \subset a\}$ is an uncountable antichain, so 1) is proved.

For the proof of 2) we are using the topological version of tightness. The algebra $A$ has countable tightness if and only if for every ultrafilter $v$ the tightness at this ultrafilter is countable, i.e., $t(v, X)=\omega$, where $X$ is the Stone space of $A$ and $t(v, X)=\omega$ means that for every $Y \subseteq X$, if $v \in \operatorname{cl}(Y)$, then there is a countable $Y^{\prime} \subset Y$ such that $v \in \operatorname{cl}\left(Y^{\prime}\right)$ (see $\left.[\mathrm{vD}],[\mathrm{H}]\right)$.

By 1) in order to check $t(A)=\omega$ it is enough to ensure that $t(u, X)=\omega$, because any other ultrafilter $v$ of $A$ has countable character and so $t(v, X)=\omega$, where $X$ is the Stone space of $A$. So suppose that there is $Y=\left\{y_{\alpha}: \alpha<\omega_{1}\right\}$ such that $c l\left(\left\{y_{\gamma}: \gamma<\alpha\right\}\right) \cap\left\{u, y_{\alpha}\right\}=\emptyset$. Let $p_{\alpha}$ have the property that $\operatorname{cl}\left(\left\{y_{\gamma}: \gamma<\alpha\right\}\right) \subseteq$ $p_{-1}^{\alpha}, y_{\alpha} \in p_{1}^{\alpha}$. Note that $p_{-1}^{\alpha}, p_{1}^{\alpha}$ may be chosen from $u^{+}$. Now, if $\alpha<\beta$, then $y_{\alpha} \in p_{1}^{\alpha} \cap p_{-1}^{\beta}$; hence $\left\{p_{\alpha}: \alpha \in \omega_{1}\right\}$ is an uncountable antichain. 
Fact 3.4. Suppose that $A$ is a Boolean algebra such that for every ultrafilter $u$ of $A$ there is a subalgebra $A_{u} \subseteq A$ such that $u$ is generated from $A_{u}$ and $P\left(A_{u}, A_{u} \cap u\right)$ is a c.c.c. notion of forcing. Then the tightness of $A, t(A)$ is countable.

Proof. Fix $u$ which is generated from $A_{u}$. Suppose $u \subseteq \operatorname{cl}\left\{y_{\alpha}: \alpha<\kappa\right\}$, where $\kappa>\omega$. By Fact 3.3 we have that $t\left(A_{u}\right)$ is countable for every ultrafilter $u$ of $A$. Thus there is $E \in[\kappa]^{\omega}$ such that $u \cap A_{u} \in \operatorname{cl}_{U l t\left(A_{u}\right)}\left\{y_{\alpha} \cap A_{u}: \alpha \in E\right\}$. We will show that $u \in \operatorname{cl}\left\{y_{\alpha} \cap A_{u}: \alpha \in E\right\}$. Take any $a \in u$; there is $b \in u \cap A_{u}$ such that $b \subseteq u$ and there is $\alpha \in E$ such that $y_{\alpha} \cap A_{u} \ni b$, so $y_{\alpha} \ni b$, which completes the proof.

Fact 3.5. There are a Boolean algebra $A$ and an ultrafilter $u$ of $A$ such that $P(A, u)$ is a c.c.c. notion of forcing and the spread of $A, s(A)$, is uncountable

Proof. Let $A$ be a Boolean algebra consisting of all finite and cofinite subsets of any uncountable cardinal $\kappa$ and let $u$ be the ultrafilter consisting of all cofinite subsets of $\kappa$. Then $P(A, u)$ is forcing with finite functions from $\kappa$ into 2 ; hence by the classical result (see $[\mathrm{K}]$ ) it is a c.c.c. notion of forcing. On the other hand $s(A)=\kappa$ (see $[\mathrm{vD}])$.

In spite of fact 3.5, we will often construct minimally generated Boolean algebras whose spread is countable or even whose hereditary density is countable. In those cases the genericity of the extending minimal element will be used. Namely, if the extending element is $(A, u)$-minimal over a sufficiently large collection of dense sets, then a lot of substructures of the smaller algebra are preserved to the extended algebra.

Below we present an example of a preservation lemma related to spread, which may be interesting by itself; it will not be used in the sequel. Recall that spread of an algebra $A$ is the supremum over the sizes of all discrete families of an algebra $A$ and recall that $\mathcal{X} \subseteq A$ is called a discrete family if and only if for every $x \in \mathcal{X}$ and for every $\mathcal{Y} \in[\mathcal{X}-\{x\}]^{<\omega}$

$$
x-\bigcup \mathcal{Y} \neq \emptyset
$$

holds.

Proposition 3.6. Let $A$ be a subalgebra of $\wp(\omega)$ and let $u$ be an ultrafilter of $A$. Let $\mathcal{X}$ be a maximal discrete family in $A$. Then there is a family $\mathcal{D}(\mathcal{X}, A, u)$, of size not greater than the size of $A$, of dense sets of $P(A, x)$ such that whenever $g$ is $(A, u)$-minimal over $\mathcal{D}(\mathcal{X}, A, u)$, then $\mathcal{X}$ is a maximal discrete family in $\langle A \cup\{g\}\rangle$.

Proof. Fix $e, f \in A$. By $\mathcal{B}$ denote the family of pairs of the form $\left(r_{-1}, r_{1}\right) \in$ $\wp(\omega) \times \wp(\omega)$ such that $r_{-1}=\omega-r_{1}$; introduce a relationship $\leq^{*}$ in $\mathcal{B} \times P(A, u)$ by putting $\left(x_{-1}, x_{1}\right) \leq^{*}\left(p_{-1}, p_{1}\right)$ if and only if $x_{i} \supseteq p_{i}$ for $i \in\{-1,1\}$. For every $p \in \mathcal{B} \cup P(A, u)$ we define $p^{*}=\left(e \cap p_{1}\right) \cup\left(f \cap p_{-1}\right)$. We will show that the set

$$
\begin{aligned}
D_{e, f}= & \left\{p \in P(A, u): \forall r \in \mathcal{B} r \leq^{*} p \text { either }(1) r^{*} \cup x_{1} \cup \ldots \cup x_{n} \supseteq x_{n+1}\right. \\
& \text { or } \left.(2) r^{*} \subseteq x_{1} \cup \ldots \cup x_{n} \text { for some } x_{1}, \ldots x_{n}, x_{n+1} \in \mathcal{X}\right\}
\end{aligned}
$$

is dense in $P(A, u)$, So fix $p \in P(A, u)$. We will find $q \leq p$ such that $q \in D_{e, f}$. Put $a=\omega-\left(p_{1} \cup p_{-1}\right), e^{\prime}=a \cap e, f^{\prime}=a \cap f$. One of the following conditions is true:

i) $e^{\prime} \cap f^{\prime} \in u$,

ii) $\omega-\left(e^{\prime} \cup f^{\prime}\right) \in u$, 
iii) $e^{\prime}-f^{\prime} \in u$,

iv) $f^{\prime}-e^{\prime} \in u$.

If i) or ii) is satisfied, then $p$ can be extended to a condition $q$ such that $q_{1} \cup q_{-1} \supseteq$ $\left(e^{\prime}-f^{\prime}\right) \cup\left(f^{\prime}-e^{\prime}\right)$ but then by De Morgan's laws we have $r^{*}=\left[r^{*} \cap(e \cap f)^{-1}\right] \cup$ $\left[r^{*} \cap(e \cap f)\right]=q^{*} \cup(e \cap f)$ for every $r \leq^{*} q$. This means that $r^{*} \in A$, so by the maximality of $X$ we have 1) or 2) from the definition of $D_{e, f}$. So we may w.l.o.g. assume that the conditions i) and ii) are false.

Suppose iii) holds. We may w.lo.g. assume that $a \subset e-f$. By maximality of $X$ in $A$ we have

$$
p^{*} \cup x_{1} \cup \ldots \cup x_{n} \supseteq x_{n+1} \text { or } p^{*} \subseteq x_{1} \cup \ldots \cup x_{n}
$$

for some $x_{1}, \ldots x_{n+1} \in X$. If the first part of the above alternative is satisfied, then we immediately obtain that for each $r \in \mathcal{B}$ such that $r \leq^{*} p$ we have satisfied 1) from the definition of the set $D_{e, f}$. Suppose, then, that the second part of the above alternative is satisfied. Again by maximality of $X$ in $A$ one of the following conditions is satisfied:

v) $a \subseteq y_{1} \cup \ldots \cup y_{m}$,

vi) $a \cup y_{1} \cup \ldots \cup y_{m} \supseteq y_{m+1}$

for some $y_{1}, \ldots, y_{m+1} \in X$.

If $\mathrm{v}$ ) is the case, then, by our assumption about $p$, for each $r \leq^{*} p$ we have $r^{*} \subseteq x_{1} \cup \ldots \cup x_{n} \cup y_{1} \cup \ldots \cup y_{m}$, so 2) from the definition of the set $D_{e, f}$ is satisfied.

If vi) is the case and $a \cap y_{m+1} \in u$, then for $q$ such that $q_{1}=p_{1} \cup\left(a-y_{m+1}\right), q_{-1}=$ $p_{-1}$ we have $q \in P(A, u)$ and for each $r \leq^{*} q, r^{*} \subseteq x_{1} \cup \ldots \cup x_{n} \cup y_{m+1}$. So 2) from the definition of $D_{e, f}$ is satisfied in this case.

If vi) is the case and $a \cap y_{m+1} \in u^{+}$, then for $q$ such that $q_{1}=p_{1} \cup\left(a \cap y_{m+1}\right), q_{-1}=$ $p_{-1}$ we have $q \in P(A, u)$ and for each $r \leq^{*} q, r^{*} \cup y_{1} \cup \ldots \cup y_{m} \supseteq y_{m+1}$. So 1) from the definition of $D_{e, f}$ is satisfied in this case.

The case when iv) holds is similar. Hence $D_{e, f}$ is dense for every $e, f \in A$. To finish the proof of the lemma note that any element of $\langle A \cup\{g\}\rangle$ is of the form $g^{*}$ for some $e, f \in A$; hence if we put $\mathcal{D}(X, A, u)=\left\{D_{e, f}: e, f \in A\right\}$, then whenever $g$ is minimal over $\mathcal{D}(X, A, u)$ for any $a \in\langle A \cup\{g\}\rangle$ we have

$$
a \cup x_{1} \cup \ldots \cup x_{n} \supseteq x_{n+1} \text { or } a \subseteq x_{1} \cup \ldots \cup x_{n}
$$

for some $x_{1}, \ldots, x_{n+1} \in \mathcal{X}$. This completes the proof of the maximality of $X$ in $\langle A \cup\{g\}\rangle$.

The above lemma can be generalized to the situation when $X$ is a maximal discrete family in some bigger algebra $A_{0} \supseteq A$ and $u$ generates an ultrafilter in $A_{0}$.

Now let us introduce another version of the forcing $P(A, u)$ which will be relevant to finite products of forcings $P(A, u)$.

Definition 3.7. Let $A$ be a Boolean algebra and $F$ a filter of $A$. Then

$$
\begin{aligned}
& P(A, F, n)=\left\{p \in A^{n}: p=\left(p_{1}, p_{2}, \ldots p_{n}\right), \forall i<j \leq n p_{i} \cap p_{j}=\emptyset \& \bigcup_{i \leq n} p_{i} \notin F\right\} . \\
& \left(p_{1}, \ldots, p_{n}\right) \leq\left(q_{1}, \ldots, q_{n}\right) \text { if and only if } p_{i} \supseteq q_{i} \text { for all } i \leq n .
\end{aligned}
$$

Note that the forcings $P(A, F)$ and $P(A, F, 2)$ are the same.

Lemma 3.8. Let $A$ be a Boolean algebra and $u$ an ultrafilter on $A$ and let $n<\omega$. There is an order preserving $\sigma: P\left(A, u, 2^{n}\right) \rightarrow P^{n}(A, u)$, where $P^{n}(A, u)$ denotes 
the product of $n$ copies of the forcing $P(A, u)$, such that the range of $\sigma$ is dense in $P^{n}(A, u)$. In particular if $P\left(A, u, 2^{n}\right)$ satisfies the c.c.c., then $P^{n}(A, u)$ satisfies the c.c.c.

Proof. It is enough to show that for each $n<\omega$ we have an order preserving function $\sigma_{n+1}: P\left(A, u, 2^{n+1}\right) \rightarrow P\left(A, u, 2^{n}\right) \times P(A, u, 2)$, with a dense range, as then the application of the composition of those functions gives an order preserving $\sigma: P\left(A, u, 2^{n}\right) \rightarrow P^{n}(A, u, 2)$, and it is enough to note that $P(A, u)=P(A, u, 2)$.

So define $\sigma_{n+1}\left(p_{1}, \ldots p_{2^{n+1}}\right)$ for $\left(p_{1}, \ldots p_{2^{n+1}}\right) \in P\left(A, u, 2^{n+1}\right)$ as

$$
\left(p_{1} \cup p_{2}, p_{3} \cup p_{4}, \ldots, p_{2^{n}-1} \cup p_{2^{n}}\right) \times\left(\bigcup\left\{p_{i}: i \text { is } \operatorname{Odd}\right\}, \bigcup\left\{p_{i}: i \text { is Even }\right\}\right) .
$$

$\sigma$ is order preserving because $a \subseteq b, c \subseteq d$ implies $a \cup c \subseteq b \cup d$.

Now let us see that the range of $\sigma_{n+1}$ is dense in $P\left(A, u, 2^{n}\right) \times P(A, u, 2)$. For any condition in $P\left(A, u, 2^{n}\right) \times P(A, u, 2)$ we can find a stronger condition of the form $\left(s_{1}, \ldots, s_{2^{n}}\right) \times\left(q_{1}, q_{2}\right)$ such that $\bigcup_{i \leq 2^{n}} s_{i}=q_{1} \cup q_{2}$. Do the calculation:

$$
\begin{aligned}
\sigma\left(s_{1}\right. & \left.\cap q_{1}, s_{1} \cap q_{2}, s_{2} \cap q_{1}, s_{2} \cap q_{2}, \ldots, s_{2^{n}} \cap q_{1}, s_{2^{n}} \cap q_{2}\right) \\
& =\left(s_{1} \cap\left(q_{1} \cup q_{2}\right), \ldots, s_{2^{n}} \cap\left(q_{1} \cup q_{2}\right)\right)\left(q_{1} \cap \bigcup_{i \leq 2^{n}} s_{i}, q_{2} \cap \bigcup_{i \leq 2^{n}} s_{i}\right) \\
& =\left(s_{1}, \ldots s_{2^{n}}\right) \times\left(q_{1}, q_{2}\right) .
\end{aligned}
$$

So the range of $\sigma_{n+1}$ is dense, as required.

\section{Hereditary separable Boolean algebras WITHOUT SMALL HOMOMORPHIC IMAGES}

In this section we provide constructions of minimally generated algebras where minimal extensions are obtained by forcing. The purpose of using forcing is to preserve some properties of subsets of the initial algebra in the extended algebra. For every subset $X$ of a given kind we associate a collection of dense subsets of the extending forcing $P(A, u)$ in such a way that if $g$ is $(A, u)$-minimal over this collection, then a given property of $X$ is preserved in $\langle A \cup\{g\}\rangle$.

Definition 4.1. Let $A$ be a Boolean algebra and let $I, J$ be proper ideals in $A$ such that $J \subset I$. We say that $X$ is a splitting $\pi$-base for $(A, I, J)$ if and only if $X \subset A-J$ and for every $a \in A-I$ there are $x_{1}, x_{2} \in X$ such that $x_{1}, x_{2} \subseteq a$ and $x_{1} \cap x_{2}=\emptyset$.

Note that the notions of a splitting $\pi$-base for $(A, I, J)$ and $\pi$-base in $A / J$ are not equivalent even when $A / J$ has no atoms and $I=J$. In the definition of a splitting $\pi$-base we require $x_{i} \subseteq a$ which is stronger than $\left[x_{i}\right]_{J} \subseteq[a]_{J}$.

Lemma 4.2. Suppose that $A \subseteq A_{0}$ are Boolean algebras which are subalgebras of $\wp(\omega)$. Let $u$ be an ultrafilter of $A$ which generates a maximal ultrafilter in $A_{0}$. Let $I \supseteq J$ be proper ideals of $A_{0}$. Suppose that $X \subseteq A_{0}$ is a splitting $\pi$-base for $\left(A_{0}, I, J\right)$. Then there is a family $\mathcal{E}\left(X, A_{0}, A, u, I, J\right)$ of dense subsets of $P(A, u)$ of size not greater than the size of $A_{0}$, such that whenever $g$ is $(A, u)$-minimal over $\mathcal{E}\left(X, A_{0}, A, u, I, J\right)$, then $X$ is a splitting $\pi$-base for $\left(B,\langle I\rangle_{I d}^{B},\langle J\rangle_{I d}^{B}\right)$, where $B=\langle A \cup\{g\}\rangle$. 
Proof. Fix any elements $e, f \in A$ and $p \in P(A, u), p=\left(p_{-1}, p_{1}\right)$ and define $r^{*}=$ $\left(r_{1} \cap e\right) \cup\left(r_{-1} \cap f\right)$ for any pair $r=\left(r_{-1}, r_{1}\right)$ of disjoint subsets of $\omega$. Also let $\underline{g}$ denote a name for the pair $\left(g^{-1}, g\right)$. We will show that

$$
\begin{aligned}
& D_{e, f}=\left\{p \in P(A, u) \text { : either } p \Vdash \underline{g}^{*} \in\langle J\rangle_{B}^{I d}\right. \\
& \left.\quad \text { or } \exists x_{1}, x_{2} \in X \quad x_{1} \cap x_{2}=\emptyset, p \Vdash x_{1}, x_{2} \subseteq \underline{g}^{*}\right\}
\end{aligned}
$$

is a dense subset of $P(A, u)$. This will be enough, since any element of $B$ is of the form $\underline{g}^{*}$ for some $e, f$ in $A$.

Take $p \in P(A, u)$. We will find $q \leq p$ from $D_{e, f}$. Consider $a=(e \cup f)-$ $\left((e-f) \cap p_{-1} \cup(f-e) \cap p_{1}\right)$. If $a \in J$, we are done since $p \| g^{*} \subseteq a$. Otherwise, using the definition of a splitting $\pi$-base, we can construct by induction three disjoint elements of $X$ included in $a$. So choose two of them $x_{1}, x_{2}$ which are not in $u$. Here we used the fact that $u$ generates an ultrafilter in $A_{0}$.

Put $q=\left(p_{-1} \cup\left[\left(x_{1} \cup x_{2}\right) \cap(f-e)\right], p_{1} \cup\left[\left(x_{1} \cup x_{2}\right) \cap(e-f)\right]\right)$. First we will prove that $q \in P(A, u)$. Since $x_{1}, x_{2}$ are not from $u$, we only have to prove that $\left(p_{1} \cap(f-e)\right) \cap\left(x_{1} \cup x_{2}\right)=p_{-1} \cap(e-f) \cap\left(x_{1} \cup x_{2}\right)=\emptyset$. This follows from the fact that $x_{1} \cup x_{2} \subseteq a$ and the fact that, by the definition, $a$ is disjoint from the sets $p_{1} \cap(f-e)$ and $p_{-1} \cap(e-f)$. Now we are left with the proof that $q \Vdash x_{1}, x_{2} \subseteq \underline{g}^{*}$. Note that $\left(x_{1} \cup x_{2}\right) \cap(e-f \cup f-e) \subseteq \underline{g}^{*}$, so $q \Vdash\left(x_{1} \cup x_{2}\right) \cap(e-f \cup f-e) \subseteq \underline{g}^{*}$ but also $q \Vdash e \cap f \subseteq \underline{g}^{*}$, so $q$ is as required.

Lemma 4.3. Suppose that $u$ is an ultrafilter of a Boolean algebra $A$ and $J$ is an ideal of $A$ such that $J \subseteq u^{+}$. Suppose that $u / J$ is a nonprincipal ultrafilter of $A / J$. Then $u^{+}-J$ is a splitting $\pi$-base for $\left(A, u^{+}, J\right)$.

Proof. Obviously $\left(u^{+}-J\right) \cap J=\emptyset$. Take $a \in A-u^{+}=u$. Since $u / J$ is a nonprincipal ultrafilter there are $y_{1}, y_{2} \in u$, such that $y_{1} \subseteq y_{2} \subseteq a$ and $\left[y_{1}\right]_{J} \subset\left[y_{2}\right]_{J} \subset[a]_{J}$. Now $x_{1}=y_{2}-y_{1}, x_{2}=a-y_{2}$ are in $u^{+}-J$ and they are disjoint and included in $a$.

Lemma 4.4. Suppose that $A$ is a hereditarily separable Boolean algebra all of whose ultrafilters are countably generated. Then for every proper ideal $J \subseteq A$ there is a countable splitting $\pi$-base $D \subseteq A$ for $(A, I, J)$, where $I=\left\langle\left\{a:[a]_{J} \in A t(A / J)\right\}\right\rangle_{A}^{I d}$.

Proof. Using the fact that the algebra is hereditary separable if and only if its every quotient $A / J$ has a countable dense subalgebra (this is the result from [Shap], see [vD] or $[\mathrm{H}]$ ), find a countable $C \subseteq A$ such that $C / J$ is dense in $A / J$.

For every $c \in C$ find an ultrafilter $u_{c}$ such that $c \in u_{c}, J \subseteq u_{c}^{+}$. Let $\{a(c, n)$ : $n<\omega\}$ be a countable base of $u_{c}$ consisting of subsets of $c$. We will show that $D=\{a(c, n): c \in C, n<\omega\}$ is a splitting $\pi$-base for $(A, I, J)$.

Suppose that $[a]_{J} \notin A t(A / J)$, so there are disjoint $a_{1}, a_{2} \in A-J$, included in $a$. Find $c_{i} \in C$ such that $\left[c_{i}\right]_{J} \subseteq\left[a_{i}\right]_{J}$ for $i=1,2$. Since $c_{i}-a_{i} \in J \subseteq u_{c_{i}}^{+}$, there are $n_{1}, n_{2}<\omega$ such that $a\left(c_{i}, n_{i}\right) \subseteq a_{i} \subseteq a$, for $i=1,2$, which completes the proof of the lemma.

Lemma 4.5. Suppose that $A$ is a hereditary separable Boolean algebra. Then every Boolean algebra $B \supseteq A$ which is countably generated over $A$ is hereditary separable as well.

Proof. It is enough to prove the lemma in the case when $B$ is one generated over $A$, i.e., $B=A\langle a\rangle$. 
This is clear, if we recall that $U l t(A\langle a\rangle) \subseteq U l t(A) \times 2$, and that an algebra is hereditary separable if and only if every subspace of its Stone space is topologically separable.

Lemma 4.6. If $h d(A)=\omega$, then $P \| h d(A)=\omega$ for a $\sigma$-centered $P$.

Proof. Suppose the opposite, so $P \Vdash\left\{\dot{a}_{\xi}: \xi<\omega_{1}\right\} \subseteq A$ is a left-separated sequence. Now we can find a centered $\left(p_{\xi}: \xi \in \omega_{1}\right) \subseteq P$ and $\left\{a_{\xi}: \xi<\omega_{1}\right\}$ such that $p_{\xi} \Vdash \check{a}_{\xi}=\dot{a}_{\xi}$. Since $h d(A)=\omega$, there are a finite $F \in \omega_{1}$ and $\eta \in \min F$, such that $\bigcap_{\xi \in F} a_{\xi}^{-1} \cap a_{\eta}=\emptyset$. But as $p_{\xi}$ 's were centered, there is $p \leq p_{\eta}, p_{\xi}$, for $\xi \in F$, so $p \Vdash\left\{\dot{a}_{\xi}: \xi \in \omega_{1}\right\}$ is not left separated, a contradiction. This completes the proof of the lemma. $\square$

Theorem 4.7. Let $\lambda$ be a cardinal such that $\lambda^{\omega}=\lambda$. Let $F n(\lambda \times \omega, 2)$ be the forcing consisting of finite partial functions from $\lambda \times \omega$ into 2 with the inverse inclusion as the order. Then in $V^{F n(\lambda \times \omega, 2)}$ there is a hereditary separable Boolean algebra $A_{\lambda}$ whose every infinite homomorphic image has size $\lambda$.

Proof. Since the forcing $F n(\lambda \times \omega, 2)$ is equivalent to its square, we may w.l.o.g. assume that $\left(2^{\omega}\right)^{V}=\lambda$.

Let $\left(X_{\alpha}: \alpha<\omega_{1}\right)$ be such a strictly increasing sequence of subsets of $\lambda$ that $\lambda=\bigcup_{\alpha<\omega_{1}} X_{\alpha}$ and $X_{\alpha+1}-X_{\alpha}$ is of size $\lambda$.

Put $M_{\alpha}=V^{F n\left(X_{\alpha} \times \omega, 2\right)}$ where $F n(Y, 2)$ denotes a subforcing of $F n(\lambda \times \omega, 2)$ consisting of these functions whose domains are included in $Y$.

Note in $V^{F n(\lambda \times \omega, 2)}$ the following facts:

1a) $\left\{M_{\alpha}: \alpha<\omega_{1}\right\}$ is a sequence of models of ZFC.

1b) $\wp(\omega)=\bigcup_{\alpha<\omega_{1}} \wp(\omega) \cap M_{\alpha}$ and for each $\alpha \leq \beta$ we have $M_{\alpha} \subseteq M_{\beta}$.

1c) For each $X \subseteq \omega_{1}$ the set $S_{X}=\left\{\alpha<\omega_{1}: X \cap \alpha \in M_{\alpha}\right\}$ is stationary in $\omega_{1}$.

Now define in $V^{F n(\lambda \times \omega, 2)}$

2a) $\left\{T_{\alpha}: \alpha \leq \omega_{1}\right\}$, a sequence of acceptable trees such that $h t\left(T_{\alpha}\right)=\alpha$.

2b) $T_{\alpha} \in M_{\alpha}$ for $\alpha<\omega_{1} . T_{\alpha} \prec T_{\beta}$ for $\alpha<\beta<\omega_{1} . T_{\omega}=2^{<\omega}$.

2c) If $\alpha$ is limit, then $T_{\alpha}=\bigcup_{\beta<\alpha} T_{\beta}$. If $\alpha=\beta+1$, then

$T_{\alpha}=T_{\beta} \cup\left\{b \frown i: b\right.$ is a maximal branch of $\left.T_{\alpha}, b \in M_{\alpha}, i \in\{-1,1\}\right\}$.

We have to note that $\bigcup_{\beta<\alpha} T_{\beta} \in M_{\alpha}$ : the definitions of $T_{\beta}$ 's are absolute and $\left(M_{\beta}: \beta<\alpha\right) \in M_{\alpha}$, so $\left.2 \mathrm{~b}\right)$ is satisfied.

Claim 1. $T_{\omega_{1}}=2^{<\omega_{1}}$. In particular all maximal branches of $T_{\omega_{1}}$ are uncountable.

Proof. Suppose $b \subseteq T_{\omega_{1}}$ is a countable maximal branch. Since $T_{\omega_{1}}$ is a union of an increasing sequence of trees, there is $\alpha<\omega_{1}$ such that $b \subseteq T_{\alpha}$, and obviously $b$ is a maximal branch of $T_{\beta}$ for each $\alpha \leq \beta \leq \omega_{1}$.

Now $b \in\left[\omega_{1}\right]^{\aleph_{0}}$, so use 1c) to find $\gamma \geq \alpha$ such that $b \in M_{\gamma}$; now by $2 \mathrm{c}$ ) we have $b^{\frown} i \in T_{\gamma+1} \subseteq T_{\omega_{1}}$ and this contradicts the maximality of $b$ in $T_{\gamma+1}$ and $T_{\omega_{1}}$.

Now define in $V^{F n(\lambda \times \omega, 2)}$

3a) $\left\{M_{X}^{\alpha}: X \in[\lambda]^{\aleph_{0}} \cap M_{\alpha}\right\}$, a sequence of models of ZFC,

by

$$
M_{X}^{\alpha}=V^{F n\left(\left(X_{\alpha} \times \omega\right) \cup\left(i_{\alpha} "(X) \times \omega\right), 2\right)}
$$

where $i_{\alpha}$ is a bijection between $\lambda$ and $X_{\alpha+1}-X_{\alpha}$ belonging to $M_{\alpha}$. Then we have the following: 
3b) For each $X \subseteq Y, X, Y \in[\lambda]^{\aleph_{0}} \cap M_{\alpha}$ we have $M_{\alpha} \subseteq M_{X}^{\alpha} \subseteq M_{Y}^{\alpha} \subseteq M_{\alpha+1}$ and $M_{\alpha+1} \cap[\lambda]^{\aleph_{0}}=\bigcup\left\{M_{X}^{\alpha} \cap[\lambda]^{\aleph_{0}}: X \in[\lambda]^{\aleph_{0}} \cap M_{\alpha}\right\}$.

3c) For each $\alpha<\omega_{1}$ and for each $X \in \wp(\lambda) \cap M_{\alpha+1}$ the set

$$
R_{X}=\left\{Y \in[\lambda]^{\aleph_{0}} \cap M_{\alpha}: Y \cap X \in M_{Y}^{\alpha}\right\}
$$

is stationary in $[\lambda]^{\aleph_{0}}$.

Before we define the algebras, we find in $M_{\alpha}$, a 1-1, onto function $\xi: B\left(T_{\alpha}\right) \rightarrow \lambda$, where $B\left(T_{\alpha}\right)$ denotes the collection of maximal branches of $T_{\alpha}$. We want the generators and algebras to satisfy the following:

4a) $\left\{a_{t}: t \in T_{\omega_{1}}\right\}$ is a sequence of subsets of $\omega$.

4b) $A_{\alpha}=\left\langle\left\{a_{t}: t \in T_{\alpha}\right\}\right\rangle \in M_{\alpha}, A_{\alpha}$ is a $T_{\alpha}$-algebra. If $\alpha<\beta$, then $A_{\alpha} \prec A_{\beta}$.

4c) If $\omega \leq \alpha<\omega_{1}, b$ is a maximal branch of $T_{\alpha}$ belonging to $M_{\alpha}$ (in particular $b \frown i \in T_{\alpha+1}$ by $\left.3 \mathrm{c}\right)$ ), then for $i=-1,1$ we have $a_{b \frown i} \in M_{\{\xi(b)\}}^{\alpha}$.

$4 d)$ Under the assumptions of $4 c), a_{b \frown i}$ is $\left(A_{b}, u_{b}\right)$-minimal over each $M_{X}^{\alpha}$ such that $\xi(b) \notin X \in[\lambda]^{\aleph_{0}}\left(u_{b}\right.$ is as in 2.8). If $Y \in[\lambda]^{\aleph_{0}} \cap M_{\alpha}$, then

$$
A\left\langle\left\{a_{b \frown i}: \xi(b) \in Y, i=-1,1\right\}\right\rangle \in M_{Y}^{\alpha} .
$$

The construction of these elements is by induction. In $M_{\{\xi(b)\}}$ define $a_{b} \frown i$ as follows. Find a dense embedding of $P\left(A_{b}, u_{b}\right)$ into $F n(\{\xi(b)\} \times \omega, 2)$. This is possible since $P\left(A_{b}, u_{b}\right)$ is a countable forcing (see $\left.[\mathrm{K}]\right)$. Thus a generic $\Gamma$ in $F n(\{\xi(b)\} \times \omega, 2)$ gives rise to a generic $\sigma(\Gamma)$ in $P\left(A_{b}, u_{b}\right)$. So let $\dot{\Gamma}$ be the canonical name for a generic set in $F n(\{\xi(b)\} \times \omega, 2)$ and define $a_{b-1}=\bigcup\left\{p_{1}: \exists p_{-1}\left(p_{-1}, p_{1}\right) \in \sigma(\dot{\Gamma})\right\}$ and then put $a_{b \frown-1}=a_{b \frown 1}^{-1}$.

Then 4c) is satisfied. By the product lemma (see $[\mathrm{K}]$ ), the restriction of the generic in $F n(\lambda \times \omega, 2)$ to $F n(\{\xi(b)\} \times \omega, 2)$ is a generic over $V^{F n(\lambda-\{\xi(b)\}, 2)}$, so $a_{b \frown i}$ is $\left(A_{b}, u_{b}\right)$-minimal over $M_{X}^{\alpha}$ for $X$ such that $\xi(b) \notin X$. So the first part of $4 \mathrm{~d})$ is satisfied.

Now if $Y \in[\lambda]^{\aleph_{0}} \cap M_{\alpha}$, then $\left\{a_{b-i}: \xi(b) \in Y\right\} \in M_{Y}^{\alpha}$, since the sequence of names for $a_{b \frown i}$, belongs to $M_{\alpha}$ and the names involve only conditions of $F n(Y \times \omega, 2)$. So 4d) is satisfied.

We note that by the construction $A_{\omega_{1}}$ is a $T_{\omega_{1}}$-algebra. We will prove that $A=A_{\omega_{1}}=\bigcup_{\alpha<\omega_{1}} A_{\alpha}$ is a hereditary separable Boolean algebra with the hereditary weight equal to $\lambda$.

It follows from claim 1 and fact 2.11, that each ultrafilter of $A_{\omega_{1}}$ has uncountable character, but we need to prove more, namely that $h w\left(A_{\omega_{1}}\right)=\lambda$.

So now, let us prove that $A / J$ is finite or of cardinality $\lambda$ for each ideal $J \subseteq A$. Assume then that $A / J$ is infinite. Let $b$ be a maximal branch of $T_{\omega_{1}}$ such that $J \subseteq u_{b}^{+}$(using the notation of lemma 2.8) and $u_{b} / J$ is a nonprincipal ultrafilter in $A / J$. This branch can be found by lemma 2.8. By claim $1, b$ is uncountable. Since $u_{b} / J$ is a nonprincipal ultrafilter in $A / J$ and $A_{b}$ generates $u_{b}$, where $A_{b}=\left\langle u_{b}\right\rangle_{A_{\omega_{1}}}$ we have that $\left(u_{b} \cap A_{b}\right) /\left(J \cap A_{b}\right)$ is a nonprincipal ultrafilter in $A_{b} / J \cap A_{b}$. Note that by the standard closure argument, the set

$C=\left\{\alpha:\left(u_{b} \cap A_{b \mid \alpha}\right) /\left(J \cap A_{b \mid \alpha}\right)\right.$ is a nonprincipal ultrafilter in $\left.\left(A_{b \mid \alpha}\right) /\left(J \cap A_{b \mid \alpha}\right)\right\}$

contains a club subset of $\omega_{1}$, where $A_{b \mid \alpha}=\left\langle\left\{a_{t}: t \in b, \operatorname{dom}(t) \subseteq \alpha\right\}\right\rangle$. The above follows from the fact that the algebras $A_{b \mid \alpha}$ are countable. Also the set

$$
D=\left\{\alpha: b \mid \alpha \subseteq T_{\alpha}\right\}
$$


contains a club subset of $\omega_{1}$. Now use 1c) to find $\alpha \in C \cap D$ such that $J \cap A_{b \mid \alpha}, b \mid \alpha \in$ $M_{\alpha}$. By 2 a) $h t\left(T_{\alpha}\right)=\alpha$, so $b \mid \alpha$ is a maximal branch of $T_{\alpha}$.

Note that $X=u_{b}^{+} \cap A_{b \mid \alpha}-\left(J \cap A_{b \mid \alpha}\right)$ is a splitting $\pi$-base for $\left(A_{b \mid \alpha}, u_{b}^{+} \cap A_{b \mid \alpha}, J\right)$ by lemma 4.3. Also $X \in M_{\alpha}$. Now, note that each $a_{t}$ for $t$ such that $b|\alpha=t| \alpha$ is minimal over $M_{\alpha} \ni X$, so we can inductively prove, using lemma 4.2 , that $X$ stays as a splitting $\pi$-base for $\left(B,\left\langle u_{b} \cap A_{b \mid \alpha}\right\rangle_{B}, J \cap B\right)$, where $B=\left\langle\left\{a_{t}: t|\alpha=b| \alpha\right\}\right\rangle$. Since $M_{\alpha} \models 2^{\omega}=\lambda$, by 2c) in $M_{\alpha+\omega}$ we have $\lambda$-many $t^{\prime}$ 's in $T_{\alpha+\omega+1}$ such that $t|\alpha=b| \alpha$; call them $\left\{t_{\xi}: \xi<\lambda\right\}$, i.e., each $t_{\xi}$ has height $\alpha+\omega+1$ and $t_{\xi}=b_{\xi} 1$, for distinct maximal branches $b_{\xi}$ of $T_{\alpha+\omega}$ such that $b_{\xi}|\alpha=b| \alpha$.

So we are left with noting that if $\eta<\xi<\lambda$, then $\left[a_{t_{\eta}}\right]_{J} \neq\left[a_{t_{\xi}}\right]_{J}$. Actually it is enough to prove that $\left[a_{t_{\eta}} \triangle a_{t_{\xi}}\right]_{K} \neq \emptyset$, where $K=\left\langle u_{b}^{+} \cap A_{b \mid \alpha}\right\rangle_{B}^{i d}$ because then there is $x \in X$ such that $x \subseteq a_{t_{\eta}} \triangle a_{t_{\xi}}$, and $X \cap J=\emptyset$.

If there is $a \in u_{b \mid \alpha}^{+}$such that $a_{t_{\eta}}-a=a_{t_{\xi}}-a$, then since $b \mid \alpha \subseteq b_{\eta}, b_{\xi}$, we conclude that $a_{t_{\eta}}-a$ is minimal over $u_{b_{\eta}}$ and $a_{t_{\xi}}-a$ is minimal over $u_{b_{\eta}}$, but it is impossible that one element is minimal over two distinct ultrafilters.

Now let us turn to the proof of the hereditary separability. First we will prove, by induction on $\alpha<\omega_{1}$, that each $A_{\alpha}$ is a hereditary separable Boolean algebra; then we will prove that $A_{\omega_{1}}$ is itself hereditary separable. So let us concentrate now on the first task.

Since limit stages have countable cofinality, they are trivial. So suppose that $A_{\alpha}$ is hereditary separable, i.e., $h d\left(A_{\alpha}\right)=\omega$ and let us aim at the proof of hereditary separability of $A_{\alpha+1}$. We will work in $M_{\alpha+1}$, where $A_{\alpha+1}$ belongs by $\left.4 \mathrm{~b}\right)$. Consider an infinite quotient $A_{\alpha+1} / J$; we will find its dense countable subalgebra.

For every $X \in[\lambda]^{\aleph_{0}}$, define

$$
A_{X}=\left\langle\left\{a_{b \frown i}: \xi(b) \in X, i \in\{-1,1\}\right\} \cup A_{\alpha}\right\rangle
$$

where $\xi(b)$ is as in $4 \mathrm{c})$. Also define

$$
I_{X}=\left\langle\left\{a \in A_{X}:[a]_{J \cap A_{X}} \in \operatorname{At}\left(A_{X} / J \cap A_{X}\right)\right\}\right\rangle_{A_{\alpha+1}}^{I d} .
$$

Note that $J \cap A_{X} \subseteq I_{X}$, and $I_{X}$ is countably generated over $J \cap A_{X}$ as $A_{X}$ is hereditary separable by lemma 4.5 .

Now find a one-to-one function $f$ from $[\lambda]^{\aleph_{0}}$ into the ordinals of $\lambda$ which are limits of limit ordinals.

By lemma 4.4, for each $X \in[\lambda]^{\aleph_{0}}$, there is a countable $D_{X} \subseteq A_{X}$ which is a splitting $\pi$-base for $\left(A_{X}, I_{X}, J \cap A_{X}\right)$. Let $Z \subseteq \lambda$ code $D_{X}$ 's, i.e., define $Z$ to have the property that if $f(X)=\alpha$, then

$$
\{\{k: \alpha+n \cdot \omega+k \in Z\}: n<\omega\}=D_{X} .
$$

Note again that $\left|A t\left(A_{X} / J \cap A_{X}\right)\right| \leq \aleph_{0}$, so we can define $g:[\lambda]^{<\omega} \rightarrow[\lambda]^{\aleph_{0}}$ such that $g(F) \supseteq F$ and if $a \in A t\left(A_{F} / A_{F} \cap J\right)$ and $F \in[\lambda]^{<\omega}$, but $a \notin A t\left(A_{\alpha+1} / J\right)$, then $a \notin A t\left(A_{g(F)} / J \cap A_{g(F)}\right)$. This can be arranged since witnessing the fact that $a$ is not an atom requires only countably many witnesses, i.e., disjoint subsets of $a$ not in $J$.

Now find a club set $\mathcal{C} \subseteq[\lambda]^{\aleph_{0}}$ such that for $X \in \mathcal{C}$, if $F \in[X]^{<\omega}$, then $\left[f(g(F)), f(g(F))+\omega^{2}\right) \subseteq X$. Now apply 3c) against $Z$ and find $Y \in[\lambda]^{\aleph_{0}} \cap M_{\alpha}$ such that $Y \in \mathcal{C}$ and $Z \cap Y \in M_{Y}^{\alpha}$.

Claim 2. $A_{Y}, I_{Y} \in M_{Y}^{\alpha}$ and there is a countable splitting $\pi$-base $D \in M_{Y}^{\alpha}$ for $\left(A_{Y}, I_{Y}, J\right)$. 
Proof. Work in $M_{Y}^{\alpha}$. Let $D=\{\{k: \alpha+n \cdot \omega+k \in Z\}: \alpha$ is a limit of limit ordinals in $\lambda, \alpha \in Y, n<\omega\}$. Since $Y \in M_{\alpha} \subseteq M_{Y}^{\alpha}, Z \cap Y \in M_{Y}^{\alpha}$, we have that $D \in M_{Y}^{\alpha}$. The fact that $Y \in \mathcal{C}$ implies that, for each $a \notin A t\left(A_{Y} / J \cap A_{Y}\right)$, there are disjoint $d_{1}, d_{2} \in D$ such that $d_{1}, d_{2} \subseteq a$. Also $D \cap J=\emptyset$, so $D$ is a splitting $\pi$-base for $\left(A_{Y}, I_{Y}, J \cap A_{Y}\right)$.

Note that the formula "there are infinitely many disjoint $d$ 's in $D$ below $x$ " in one free variable $x$ defines exactly $A_{Y}-I_{Y}$, since $Y \in \mathcal{C}$ and if $a \in I_{Y}$, then there cannot be infinitely many disjoint elements below $a$ from outside of $J$. So $I_{Y} \in M_{Y}^{\alpha}$ and this completes the proof of the claim.

Note that by $4 \mathrm{~d}$ ) since $\xi(b) \notin Y$, if $a_{b \frown i} \notin A_{Y}$, and so, all the generators of $A_{\alpha+1}$ over $A_{Y}$ are $\left(A_{b}, u_{b}\right)$-minimal over $M_{Y}^{\alpha}$, then using lemma 4.2 inductively we prove that $D$ is a splitting $\pi$-base for $\left(A_{\alpha+1},\left\langle I_{Y}\right\rangle, J\right)$.

Now note that $D$ and atoms of $A_{Y} / J \cap A_{Y}$ generate a countable dense subalgebra of $A_{\alpha+1} / J$. If $a \in A_{\alpha+1}-\left\langle I_{Y}\right\rangle$, then there is $d \subseteq a, d \in D$. If $a \in\left\langle I_{Y}\right\rangle$, then $a$ is covered by finitely many atoms of $A_{Y} / A_{Y} \cap J$; since those atoms are real atoms of $A_{\alpha+1} / J$ by the choice of $Y \in \mathcal{C}$, one of them has to be below a modulo $J$. So $h d\left(A_{\alpha+1}\right)=\omega$ in $M_{\alpha+1}$, so $h d\left(A_{\alpha+1}\right)=\omega$ by 4.6 .

Now we are left with the proof that $A_{\omega_{1}}$ is hereditary separable. We will find a countable dense subalgebra in any homomorphic image of $A_{\omega_{1}}$. So, take an ideal $J \subseteq A_{\omega_{1}}$. For each limit of limit ordinals $\xi$ in $\omega_{1}$, define

$$
I_{\xi}=\left\langle\left\{a \in A_{\xi}:[a]_{J \cap A_{\xi}} \in \operatorname{At}\left(A_{\xi} / J \cap A_{\xi}\right)\right\}\right\rangle .
$$

Note that $J \cap A_{\xi} \subseteq I_{\xi}$ and $I_{\xi}$ is countably generated over $J \cap A_{\xi}$; as $A_{\xi}$ is hereditary separable, $A_{\xi} / J \cap A_{\xi}$ has at most countably many atoms.

As $h d\left(A_{\xi}\right)$ and $A_{\xi}$ have all ultrafilters countably generated (by lemma 4.4 and the fact that $A_{\xi}$ is a $T_{\xi}$-algebra and $h t\left(T_{\xi}\right)=\xi$ by $\left.\left.2 \mathrm{a}\right)\right)$ there is a countable $D_{\xi} \subseteq A_{\xi}$ which is a splitting $\pi$-base for $\left(A_{\xi}, I_{\xi}, J \cap A_{\xi}\right)$. Let $X \subseteq \omega_{1}$ code $D_{\xi}$ 's, i.e., define $X$ to have the property that

$$
\{\{k: \alpha+n \cdot \omega+k \in X\}: n<\omega\}=D_{\xi} .
$$

Note again that $\left|A t\left(A_{\xi} / J \cap A_{\xi}\right)\right| \leq \aleph_{0}$, so we can find $g: \omega_{1} \rightarrow \omega_{1}$ such that $g(\xi) \geq \xi$ and if $a \in A t\left(A_{\xi} / J \cap A_{\xi}\right)$, but $a \notin A t\left(A_{\omega_{1}} / J\right)$, then $a \notin A t\left(A_{g(\xi)} / J \cap A_{g(\xi)}\right)$. The argument is as before.

Now find a club set $C$ in $\omega_{1}$, consisting of limits of countable limit ordinals such that for $\xi \in C$ if $\eta<\xi$, then $g(\eta)<\xi$. Now apply 1c) against $X$ and find $\xi \in C$ such that $X \cap \xi \in M_{\xi}$. As before we prove

Claim 3. $A_{\xi}, I_{\xi} \in M_{\xi}$ and there is a countable $D \in M_{\xi}$ such that $D$ is a splitting $\pi$-base for $\left(A_{\xi}, I_{\xi}, J\right)$.

Proof. The proof is similar to the proof of the previous claim. One needs to replace $Z$ with $X \cap \xi$ and $Y$ with $\xi$.

Now note that by $4 \mathrm{~d})$ all generators of $A_{\omega_{1}}$ over $A_{\xi}$ are $\left(A_{b}, u_{b}\right)$-minimal over $M_{\xi}$ for some branch $b$ of $T_{\xi}$, so using lemma 4.2 inductively we prove that $D$ is a splitting $\pi$-base for $\left(A_{\xi},\left\langle I_{\xi}\right\rangle_{A_{\omega_{1}}}, J\right)$. As before we argue that $D$ and the atoms of $A_{\xi} / J \cap A_{\xi}$ generate a countable dense subalgebra of $A_{\omega_{1}} / J$ which completes the proof of the lemma.

As a corollary from the proof of the previous theorem we obtain the following 
Lemma 4.8. Suppose that $2^{\omega}=\lambda$ and that there are the following sequences:

1a) $\left\{M_{\alpha}: \alpha<\omega_{1}\right\}$, a sequence of models of a sufficiently large fragment of ZFC.

1b) $\wp(\omega)=\bigcup_{\alpha<\omega_{1}} \wp(\omega) \cap M_{\alpha}$ and for each $\alpha \leq \beta$ we have $M_{\alpha} \subseteq M_{\beta}$.

1c) For each $X \subseteq \omega_{1}$ the set $S_{X}=\left\{\alpha<\omega_{1}: X \cap \alpha \in M_{\alpha}\right\}$ is stationary in $\omega_{1}$.

2a) $\left\{T_{\alpha}: \alpha<\omega_{1}\right\}$, a sequence of acceptable trees such that $h t\left(T_{\alpha}\right)=\alpha$.

2b) $T_{\alpha} \in M_{\alpha}$ for $\alpha<\omega_{1} . T_{\alpha} \prec T_{\beta}$ for $\alpha<\beta<\omega_{1} . T_{\omega}=2^{<\omega}$.

2c) If $\alpha$ is limit, then $T_{\alpha}=\bigcup_{\beta<\alpha} T_{\beta}$. If $\alpha=\beta+1$, then $T_{\alpha}=T_{\beta} \cup\{b \frown i$ : $b$ is a maximal branch of $\left.T_{\alpha}, b \in M_{\alpha}, i \in\{-1,1\}\right\}$.

3a) $\left\{M_{X}^{\alpha}: X \in[\lambda]^{\aleph_{0}} \cap M_{\alpha}\right\}$, a sequence of models of a sufficiently large fragment of $Z F C$.

3b) For each $X \subseteq Y, X, Y \in[\lambda]^{\aleph_{0}} \cap M_{\alpha}$ we have $M_{\alpha} \subseteq M_{X}^{\alpha} \subseteq M_{Y}^{\alpha} \subseteq M_{\alpha+1}$ and $M_{\alpha+1} \cap[\lambda]^{\aleph_{0}}=\bigcup\left\{M_{X}^{\alpha} \cap[\lambda]^{\aleph_{0}}: X \in[\lambda]^{\aleph_{0}} \cap M_{\alpha}\right\}$.

3c) For each $\alpha<\omega_{1}$ and for each $X \in \wp(\lambda) \cap M_{\alpha+1}$ the set $R_{X}=\{Y \in$ $\left.[\lambda]^{\aleph_{0}} \cap M_{\alpha}: Y \cap X \in M_{Y}^{\alpha}\right\}$ is stationary in $[\lambda]^{\aleph_{0}}$.

4a) $\left\{a_{t}: t \in T_{\omega_{1}}\right\}$, a sequence of subsets of $\omega$.

4b) $A_{\alpha}=\left\langle\left\{a_{t}: t \in T_{\alpha}\right\}\right\rangle \in M_{\alpha}, A_{\alpha}$ is a $T_{\alpha}$-algebra. If $\alpha<\beta$, then $A_{\alpha} \prec A_{\beta}$.

4c) If $\omega \leq \alpha<\omega_{1}, b$ is a maximal branch of $T_{\alpha}$ belonging to $M_{\alpha}$ (in particular $b \frown i \in T_{\alpha+1}$ by 3c), then for $i=-1,1$ we have $a_{b \frown i} \in M_{\{\xi(b)\}}^{\alpha}$ for some $\xi(b) \in \lambda$. If $b_{1} \neq b_{2}$, then $\xi\left(b_{1}\right) \neq \xi\left(b_{2}\right)$.

4d) Under the assumptions of $4 c), a_{b \frown i}$ is $\left(A_{b}, u_{b}\right)$-minimal over each $M_{X}^{\alpha}$ such that $\xi(b) \notin X \in[\lambda]^{\aleph_{0}}$. If $Y \in[\lambda]^{\aleph_{0}} \cap\left[M_{\alpha}\right]$, then $A\left\langle\left\{a_{b \frown i}: \xi(b) \in Y, i=-1,1\right\}\right\rangle \in$ $M_{Y}^{\alpha}$.

5) If $A \in M_{\alpha}$ and $M_{\alpha}=h d(A)=\omega$, then $h d(A)=\omega$.

Then $A=A_{\omega_{1}}=\bigcup_{\alpha<\omega_{1}} A_{\alpha}$ is a hereditary separable Boolean algebra with the hereditary weight equal to $\lambda$.

The above lemma enables us to prove the following generalization of theorem 4.7 which will be useful in the following section.

Theorem 4.9. Suppose that $2^{\omega}=\lambda$. Let $\left(P_{\alpha}, P^{\alpha}\right)_{\alpha<\omega_{1}}$ be a finite support iteration such that

$$
P_{\alpha} \Vdash P^{\alpha}=R^{\alpha} \times F n(\lambda \times \omega, 2), \quad R^{\alpha} \text { is } \sigma \text {-centered, }
$$

Then in $V^{P_{\omega_{1}}}$, the assumptions of lemma 4.8 are satisfied.

Proof. Define $M_{\xi}=V^{P_{\xi}}$, for $\xi<\omega_{1}$. Then, using the standard argument for c.c.c. forcings we obtain 1a), 1b), 1c).

Define in $V^{P_{\alpha}}, M_{X}^{\alpha}$ as $V^{P_{\alpha} *\left(R^{\alpha} \times F n(X \times \omega, 2)\right)}$. Then using the standard argument for c.c.c. forcings $2 \mathrm{a}), 2 \mathrm{~b}), 2 \mathrm{c}$ ) are satisfied.

Now define $\left(T_{\alpha}: \alpha<\omega_{1}\right)$ as in $\left.\left.3 \mathrm{a}\right), 3 \mathrm{c}\right)$. We have to note that $\bigcup_{\beta<\alpha} T_{\beta} \in M_{\alpha}$, but the definitions of $T_{\beta}$ 's are absolute and $\left(M_{\beta}: \beta<\alpha\right) \in M_{\alpha}$, so $\left.3 \mathrm{~b}\right)$ is satisfied.

Now define the $A_{\alpha}$ 's. For this in $M_{\alpha}$, find a 1-1, onto function $\xi: B\left(T_{\alpha}\right) \rightarrow \lambda$, where $B\left(T_{\alpha}\right)$ denotes the collection of maximal branches of $T_{\alpha}$.

In $M_{\{\xi(b)\}}$ define $a_{b} \frown i$ as follows. Find a dense embedding of $P\left(A_{b}, u_{b}\right)$ into $F n(\{\xi(b)\} \times \omega, 2)$. This is possible since $P\left(A_{b}, u_{b}\right)$ is a countable forcing (see $\left.[\mathrm{K}]\right)$. Thus a generic $\Gamma$ in $F n(\{\xi(b)\} \times \omega, 2)$ gives rise to a generic $\sigma(\Gamma))$ in $P\left(A_{b}, u_{b}\right)$. So let $\dot{\Gamma}$ be the canonical name for a generic set in $F n(\{\xi(b)\} \times \omega, 2)$ and define $a_{b-1}=\bigcup\left\{p_{1}: \exists p_{-1}\left(p_{-1}, p_{1}\right) \in \sigma(\dot{\Gamma})\right\}$ and then put $a_{b}--1=a_{b \frown 1}^{-1}$.

Then $4 \mathrm{c}$ ) is satisfied. By the product lemma (see $[\mathrm{K}]$ ), the restriction of the generic in $F n(\lambda \times \omega, 2)$ to $F n(\{\xi(b)\} \times \omega, 2)$ is a generic over $V^{F n(\lambda-\{\xi(b)\}, 2)}$, so 
$a_{b-i}$ is $\left(A_{b}, u_{b}\right)$-minimal over $M_{X}^{\alpha}$ for $X$ such that $\xi(b) \notin X$. So the first part of $4 \mathrm{~d})$ is satisfied.

Now if $Y \in[\lambda]^{\aleph_{0}} \cap M_{\alpha}$, then $\left\{a_{b-i}: \xi(b) \in Y\right\} \in M_{Y}^{\alpha}$, since the sequence of names for $a_{b \frown i}$, belongs to $M_{\alpha}$ and the names involve only conditions of $F n(Y, 2)$. So $4 \mathrm{~d}$ ) is satisfied.

To prove 5), note that $P_{\alpha} \Vdash P_{\left[\alpha, \omega_{1}\right)}$ is $\sigma$-centered and apply lemma 4.6.

\section{A MOdified CONSTRUCtion OF A HEREDitARY SEPARABlE ALGEBRA WITH UNCOUNTABLE HEREDITARY WEIGHT}

The main purpose of this section is to construct a Boolean algebra of size $\omega_{1}$ with hereditary density countable, without a converging sequence, in its Stone's space, in a presence of a arbitrary negation of $\mathrm{CH}$ (up to necessary set-theoretic restrictions). Our construction will take place in a model in which there exists an algebra as constructed in section 4 . Thus we will prove the consistency of the simultaneous existence of Boolean algebras with the homomorphic cellularity relation equal to $\left\{\omega, \omega_{1}\right\}$ and $\left\{\omega, 2^{\omega}\right\}$ where $2^{\omega}$ can be any cardinal of uncountable cofinality. We will consider the following forcing notion.

Definition 5.1. Let $A$ be a Boolean algebra; then

$$
\begin{gathered}
R(A)=\left\{p=\left(p_{-1}, p_{1} ; u_{1}, \ldots, u_{n}\right):\left(p_{-1}, p_{1}\right) \in \bigcap_{i \leq n} P\left(A, u_{i}\right), \forall i \leq n u_{i} \in \operatorname{Ult}(A)\right\}, \\
\left(p_{-1}, p_{1} ; u_{1}, \ldots, u_{n}\right) \leq\left(q_{-1}, q_{1}, v_{1}, \ldots, v_{m}\right) \text { iff } \\
p_{-1} \supseteq q_{-1}, p_{1} \supseteq q_{1},\left\{u_{1}, \ldots, u_{n}\right\} \supseteq\left\{v_{1}, \ldots, v_{m}\right\} .
\end{gathered}
$$

Note that $R(A)$ does not give elements which are minimal over $A$ as we had in the case of the forcing $P(A, u)$. One can see that the set of ultrafilters which are extended by adding a generic set for $R(A)$ to the algebra $A$ is nowhere dense in the Stone space of $A$. Nevertheless, since this nowhere dense set is added generically, $R(A)$ has a lot of properties of $P(A, u)$; for example maximal discrete families in $A$ stay maximal in $A\langle g\rangle$ if $g$ is obtained from a generic set in $R(A)$ over a model containing a given discrete family. This fact will not be needed in this section and its proof which is similar to the proof of lemma 3.6 is left to the reader as an exercise. We will not analyze in detail extending algebras with the forcing $R(A)$ since the application we have in mind is not complicated. We will separately state only the following fact, and the next preservation lemma.

Fact 5.2. $R(A)$ is a $\sigma$-centered notion of forcing for any countable, atomless Boolean algebra $A$.

Proof. Note that elements with the same first two coordinates are compatible.

Lemma 5.3. Suppose that $A$ is a Boolean algebra and $J \subseteq I$ are proper ideals of $A$. Suppose that $X \subseteq A$ is a splitting $\pi$-base for $(A, I, J)$. Then there is a family $\mathcal{E}(X, A, I, J)$ of dense subsets of $R(A)$ of size not greater than the size of $A$, such that whenever $h(A)=\bigcup\left\{p_{1}: \exists p_{-1}, u_{1}, \ldots, u_{n}\left(p_{-1}, p_{1} ; u_{1}, \ldots, u_{n}\right) \in G\right\}$ where $G$ denotes the canonical name for generic in $R(A)$, then $X$ is a splitting $\pi$-base for $\left(B,\langle I\rangle_{B}^{I d},\langle J\rangle_{B}^{I d}\right)$, where $B=\langle A \cup\{h(A)\}\rangle$. 
Proof. Fix any elements $e, f \in A$ and $p \in R(A), p=\left(p_{-1}, p_{1} ; u_{1}, \ldots, u_{m}\right)$ and define $r^{*}=\left(r_{1} \cap e\right) \cup\left(r_{-1} \cap f\right)$ for any pair $\left(r_{-1}, r_{1}\right)$ of disjoint subsets of $\omega$. Also let $h$ denote a name for the pair $\left(h(A)^{-1}, h(A)\right)$. We will show that

$$
\begin{aligned}
& D_{e, f}=\left\{p \in R(A): \text { either } p \Vdash h^{*} \in\langle I\rangle_{B}^{I d}\right. \\
& \text { or } \left.\exists x_{1}, x_{2} \in X x_{1} \cap x_{2}=\emptyset, p \Vdash x_{1}, x_{2} \subseteq h^{*}\right\}
\end{aligned}
$$

is a dense subset of $R(A)$. This will be enough, since any element of $B$ is of the form $h^{*}$ for some $e, f$ in $A$.

Take $p \in R(A)$. We will find $q \leq p$ from $D_{e, f}$. Consider

$$
a=(e \cup f)-\left((e-f) \cap p_{-1} \cup(f-e) \cap p_{1}\right) .
$$

If $a \in I$, we are done since $p \|-h^{*} \subseteq a$. Otherwise, using the definition of a splitting $\pi$-base, we can construct by induction $m+2$ pairwise disjoint elements of $X$ included in $a$. So choose two of them $x_{1}, x_{2}$ which are in none of the $u_{1}, \ldots, u_{m}$.

Put $q=\left(p_{-1} \cup\left[\left(x_{1} \cup x_{2}\right) \cap(f-e)\right], p_{1} \cup\left[\left(x_{1} \cup x_{2}\right) \cap(e-f)\right] ;\left\{u_{1}, \ldots, u_{m}\right\}\right)$. First we will prove that $q \in R(A)$. Since $x_{1}, x_{2}$ are disjoint from $\left\{u_{1}, \ldots, u_{m}\right\}$ we only have to prove that $p_{1} \cap(f-e) \cap\left(x_{1} \cup x_{2}\right)=p_{-1} \cap(e-f) \cap\left(x_{1} \cup x_{2}\right)=\emptyset$. This follows from the fact that $x_{1} \cup x_{2} \subseteq a$ and the fact that by the definition, $a$ is disjoint from the sets $p_{1} \cap(f-e)$ and $p_{-1} \cap(e-f)$. Now we are left with the proof that $q \Vdash x_{1}, x_{2} \subseteq h^{*}$. Note that $\left(x_{1} \cup x_{2}\right) \cap(e-f \cup f-e) \subseteq q^{*}$, so $q^{*} \|\left(x_{1} \cup x_{2}\right) \cap(e-f \cup f-e) \subseteq h^{*}$ but also $q \Vdash e \cap f \subseteq h^{*}$ since $q \Vdash h(A)^{-1} \cup h(A)=\omega$. So $q$ is as required.

Theorem 5.4. Suppose that $\left(P_{\alpha}, P^{\alpha}\right)_{\alpha<\omega_{1}}$ is a finite support iteration of forcings such that

$$
P_{\alpha} \Vdash P^{\alpha}=R\left(\operatorname{Clop}\left(2^{\omega}\right)\right) * Q^{\alpha}, Q^{\alpha} \text { satisfies the c.c.c. }
$$

Then in $V^{P_{\omega_{1}}}$ there is a hereditary separable Boolean algebra $A$ of size $\omega_{1}$, without countably infinite homomorphic images.

Proof. By induction on $\omega \leq \alpha<\omega_{1}$, we construct $A_{\alpha}$ such that

0) $A_{\omega}=\operatorname{clop}\left(2^{\omega}\right)$.

1) $A_{\alpha} \in V^{P_{\alpha}}, A_{\alpha} \subseteq A_{\beta}$ for $\alpha \leq \beta$.

2) If $\alpha$ is a limit ordinal, then $A_{\alpha}=\bigcup_{\beta<\alpha} A_{\beta}$.

3) If $\alpha=\beta+1$, for some $\beta<\omega_{1}$, then in $V^{P_{\beta+1}}$ we define $A_{\beta+1}$ as the algebra generated over $A_{\beta}$ by the element $a_{\beta}$ where

$$
a_{\beta}=\bigcup\left\{p_{1}: \exists p_{-1}, u_{1}, \ldots, u_{n}\left(p_{-1}, p_{1} ; u_{1}, \ldots, u_{n}\right) \in G_{\beta}\right\}
$$

where $G_{\beta}$ denotes a generic set in $R\left(A_{\beta}\right)$ over $V^{P_{\beta}}$.

4) $A=A_{\omega_{1}}=\bigcup_{\alpha<\omega_{1}} A_{\alpha}$.

To prove that the construction can be carried out, we have to argue that in $V^{P_{\beta+1}}$ there is a generic in $R\left(A_{\beta}\right)$ over $V^{P_{\beta}}$. First note that nonempty clopen subsets of $2^{\omega}$ form a splitting $\pi$-base for $\left(A_{\omega},\{\emptyset\},\{\emptyset\}\right)$, so we can argue by induction, using lemma 5.3 , that those sets form a splitting $\pi$-basis for $\left(A_{\beta},\{\emptyset\},\{\emptyset\}\right)$. Thus $A_{\beta}$ is atomless; hence isomorphic to $\operatorname{clop}\left(2^{\omega}\right)$. It follows that $R\left(A_{\beta}\right)$ is isomorphic to $R\left(\operatorname{clop}\left(2^{\omega}\right)\right)$; hence the generic in $R\left(\operatorname{clop}\left(2^{\omega}\right)\right)$ over $V^{P_{\beta}}$, which exists in $V^{P_{\beta+1}}$ by the assumption of the theorem, gives rise to a generic in $R\left(A_{\beta}\right)$ over $V^{P_{\beta}}$; hence the construction can be accomplished in our model.

Claim 1. A has no countably infinite homomorphic images 
Proof. First we will note that no ultrafilter of $A_{\omega_{1}}$ is countably generated. Suppose the opposite; let $u$ have countable character. Then, as $P_{\omega_{1}}$ is an iteration of c.c.c. forcings, there is $\alpha<\omega_{1}$ such that $u \in V^{P_{\alpha}}$. Work in $V^{P_{\alpha}}$. Consider any condition $p \in P_{\left(\alpha, \omega_{1}\right]}$. Let $\beta \notin \operatorname{supp}(p)$ and put $q=p \frown\{\langle\beta,(\emptyset, \emptyset ;\{u\})\rangle\}$. Then $q \in P_{\left(\alpha, \omega_{1}\right]}$ and obviously $q$ forces that $u$ is not an ultrafilter in $A_{\beta+1}$, a contradiction.

Now, suppose that there is an ideal $J \subseteq A$ such that $A / J=C$ is countably infinite. Let $I^{\prime} \subseteq C$ be a countably generated nonprincipal maximal ideal. Let $I=\pi^{-1}\left(I^{\prime}\right)$, where $\pi: A \rightarrow C$ is the canonical epimorphism. Note that $X=A-J$ is a splitting $\pi$-base for $(A, I, J)$; this follows from lemma 4.3. Now using the standard closure argument and the fact that our iteration is an iteration with finite supports of forcings satisfying the c.c.c., we can find $\alpha<\omega_{1}$ such that $X \cap A_{\alpha}$ is a splitting $\pi$-base for $\left(A_{\alpha}, I \cap A_{\alpha}, J \cap A_{\alpha}\right)$ and $X \cap A_{\alpha}, I \cap A_{\alpha}, J \cap A_{\alpha} \in V^{P_{\alpha}}$. By lemma 5.3 used inductively we conclude that $X \cap A_{\alpha}$ is a splitting $\pi$-basis for $\left(A,\left\langle I \cap A_{\alpha}\right\rangle, J\right)$.

Now using the fact that there are no countably generated ultrafilters in $A$ we find a sequence $\left\{i_{\xi}: \xi<\omega_{1}\right\} \subseteq I$ generating $I$ over $I \cap A_{\alpha}$ such that $i_{\xi} \notin I_{\xi}$ where $I_{0}=I \cap A_{\alpha}$ and $I_{\xi}=\left\langle\left\{i_{\eta}: \eta<\xi\right\} \cup I_{0}\right\rangle_{A}^{I d}$ for $0<\xi<\omega_{1}$. We will show that for every $\xi_{1}<\xi_{2}<\omega_{1}$ we have $\pi\left(i_{\xi_{2}}\right)-\pi\left(i_{\xi_{1}}\right) \neq \emptyset$. This will prove that $A / J$ is uncountable.

Suppose $\xi_{1}<\xi_{2}$. Since $i_{\xi} \notin I_{\xi}$ we conclude that $i_{\xi_{2}}-i_{\xi_{1}} \notin I_{\xi_{1}} \supseteq I_{0}=I \cap A_{\alpha}$; hence since $X \cap A_{\alpha}$ is a splitting $\pi$-base for $\left(A,\left\langle I \cap A_{\alpha}\right\rangle, J\right)$, there is $a \in X \cap A_{\alpha}$ such that $a \notin J$ and so $a \notin I$, and $a \subseteq i_{\xi_{1}}-i_{\xi_{2}}$, i.e., $\pi\left(i_{\xi_{1}}\right)-\pi\left(i_{\xi_{2}}\right) \neq \emptyset$. This completes the proof of claim 1 .

Claim 2. The hereditary density of $A$ is countable.

Proof. We will show that every quotient $A / J$ of $A$ has a countable dense subalgebra; this is enough by a result from [Shap] (see $[\mathrm{vD}]$ or $[\mathrm{H}]$ ).

So fix an ideal $J \subseteq A$. Using the fact that our iteration is a finite support iteration of forcings satisfying the c.c.c. and the fact that each $A_{\alpha}$ is countable and applying the standard closure argument (see $[\mathrm{K}]$ ), we can find $\alpha<\omega_{1}$ such that:

a) $J \cap A_{\alpha} \in V^{P_{\alpha}}$.

b) In $V^{P_{\alpha}}$, if $a \in A t\left(A_{\alpha} /\left(J \cap A_{\alpha}\right)\right)$, then $a \in A t(A /(J \cap A))$.

c) $A_{\alpha} /\left(J \cap A_{\alpha}\right)$ is infinite.

Now $X=A_{\alpha}-J$ is a splitting $\pi$-base for $\left(A_{\alpha}, I_{\alpha}, J_{\alpha}\right)$ where

$$
I_{\alpha}=\left\langle\left\{a:[a]_{J \cap A_{\alpha}} \in \operatorname{At}\left(A_{\alpha} /\left(J \cap A_{\alpha}\right)\right)\right\}\right\rangle
$$

and by a), b), c) we have $X, I_{\alpha} \in V^{P_{\alpha}}$. So applying lemma 5.3 inductively we obtain that $X$ is a splitting $\pi$-base for $\left(A,\left\langle I_{\alpha}\right\rangle_{A}, J\right)$.

We will prove that $X$ and atoms of $A_{\alpha} / J \cap A_{\alpha}$ generate a countable dense subalgebra of $A / J$. If $a \in A-\left\langle I_{\alpha}\right\rangle$, then there is $x \in X \subseteq A-J, x \subseteq a$, by the definition of a splitting $\pi$-base. If $a \in\left\langle I_{\alpha}\right\rangle$, then there are $a_{1}, \ldots, a_{n}$ which are atoms of $A_{\alpha} / J \cap A_{\alpha}$ and $a \subseteq a_{1} \cup \ldots \cup a_{n}$. By b) we get that $\left[a_{1}\right]_{J}, \ldots,\left[a_{n}\right]_{J}$ are atoms of $A / J$, so for some $i \leq n$ we have $\left[a_{i}\right]_{J} \subseteq[a]_{J}$. Obviously $I_{\alpha}$ is countable since $A_{\alpha}$ is countable, so this completes the proof of the claim and the proof of the theorem.

Theorem 5.5. Suppose that $\lambda$ is a cardinal of uncountable cofinality. If $Z F C$ is consistent, then so is the following: $Z F C+$ there are Boolean algebras $A_{\lambda}$ and $A_{\omega_{1}}$ such that $h d\left(A_{\lambda}\right)=h d\left(A_{\omega_{1}}\right)=\omega$ and $h w\left(A_{\lambda}\right)=\lambda$ and $h w\left(A_{\omega_{1}}\right)=\omega_{1}$. 
Proof. Note that the assumptions of theorem 5.4 and theorem 4.9 can be satisfied in the same model of ZFC. For this note that, by lemma 5.2, the iteration from theorem 4.9, satisfies the assumptions of theorem 5.4.

\section{Some DERIVED ALGEBRAS}

The main purpose of this section is to derive a Boolean algebra with the cellularity homomorphic relation (see the introduction for the definition) equal to $\left\{\left(\omega_{1}, \lambda\right),(\omega, \lambda)\right\}$ from a Boolean algebra with the cellularity homomorphic relation equal to $\{(\omega, \lambda)\}$, for an uncountable cardinal $\lambda$. The construction will involve a structure of direct sums of Boolean algebras. For the convenience of the reader we state elementary definitions and propositions concerning finite direct sums (or disjoint sums, in the topological language). (Note that our notation is different than in [Kop3].)

Definition 6.1. Suppose that $A, B$ are infinite Boolean algebras. We write $A \leq_{h}$ $B$ if and only if $A$ is a homomorphic image of $B$. We write $A \leq B$ if and only if $A$ is a subalgebra of $B$.

Definition 6.2. Suppose $A$ and $B$ are Boolean algebras. Then $A \oplus B$ denotes the Boolean algebra constructed as follows: Find disjoint sets $X, Y$ such that $A$ and $B$ are fields of subsets of $X$ and $Y$ respectively. Let $A \oplus B$ be the algebra isomorphic to the field of subsets of $X \cup Y$ generated by $A \cup B$.

Lemma 6.3. Suppose that $C \leq_{h} A_{1} \oplus \ldots \oplus A_{n}$. Then there are algebras $A_{1}^{\prime}, \ldots, A_{n}^{\prime}$ such that $C=A_{1}^{\prime} \oplus \ldots \oplus A_{n}^{\prime}$ and $A_{i}^{\prime} \leq_{h} A_{i}$ for $i \leq n$.

Definition 6.4. Suppose $A_{i}$, for $i<\omega$, and $B$ are Boolean algebras and $[\omega]<\aleph_{0} \subset$ $B \subseteq \wp(\omega)$. Then $\bigoplus_{i<\omega}^{B} A_{i}$ is the Boolean algebra obtained as follows: Find disjoint sets $X_{i}$ for $i<\omega$ such that $A_{i}$ 's are fields of subsets of $X_{i}$ 's respectively. Then $\bigoplus_{i<\omega}^{B} A_{i}$ is the Boolean algebra isomorphic to the field of subsets of $\bigcup_{i<\omega} X_{i}$ consisting of elements of the form

$$
c=c\left(b, F,\left\{a_{i}: i \in F\right\}\right)=\bigcup_{i \in b} X_{i} \cup\left\{a_{i}: i \in F\right\}
$$

where $b \in B, F \in[\omega]^{<\omega}, a_{i} \in A_{i}$ for all $i \in F$.

Lemma 6.5. Suppose $A_{i}$, for $i<\omega$, and $B$ are as in definition 6.4. Suppose $C \leq_{h} \bigoplus_{i<\omega}^{B} A_{i}$. Then there is $D \leq_{h} C$ such that either $D \leq_{h} B$ or $D \leq_{h} A_{i}$ for some $i<\omega$.

Proof. Let $I$ be an ideal of $\bigoplus_{i<\omega}^{B} A_{i}$ such that the obtained quotient is equal to $C$. Let $J_{i}=\left\{b \subseteq X_{i}: \exists e \in J e \cap X_{i}=b\right\}$ for any ideal $J \subseteq \bigoplus_{i<\omega}^{B} A_{i}$.

Case 1. For all but finitely many $i<\omega, X_{i} \in I$ and for every $i<\omega$ the algebra $A_{i} / I_{i}$ is finite.

Let $K$ be the ideal generated by $I \cup\left\{X_{j}: X_{j} \notin I\right\}$. First let us prove that $\left(\bigoplus_{i<\omega}^{B} A_{i}\right) / K$ is infinite. $K$ is one generated over $I$ by $X=\bigcup\left\{X_{i}: X_{i} \notin I\right\}$, since there are only finitely many $i<\omega$ such that $X_{i} \notin I$. If $\left(\bigoplus_{i<\omega}^{B} A_{i}\right) / K$ were finite, it would mean that $\left(\bigoplus_{i<\omega}^{B} A_{i} \mid Y\right) /(I \mid Y)$ is finite, where $Y=\bigcup_{i<\omega} X_{i}-X$. But the size of every $A_{i} / I_{i}$ is finite so $\left(\bigoplus_{i<\omega}^{B} A_{i} \mid X\right) /(I \mid X)$ is finite; thus $\left(\bigoplus_{i<\omega}^{B} A_{i}\right) / I$ is finite, a contradiction. Now note that it follows from definition 6.4 that for 
every $c \in \bigoplus_{i<\omega}^{B} A_{i}$ we have $\left[c\left(b, F,\left\{a_{i}: i \in F\right\}\right)\right]_{K}=[c(b, \emptyset, \emptyset)]_{K}$. Note that if $B^{\prime}=\{c(b, \emptyset, \emptyset): b \in B\}$, then there is an isomorphism $\pi: B \rightarrow B^{\prime} \leq \bigoplus_{i<\omega}^{B} A_{i}$, so $\bigoplus_{i<\omega}^{B} A_{i} / K=B^{\prime} /\left(K \cap B^{\prime}\right)=B /\left(\pi^{-1}\left(K \cap B^{\prime}\right)\right)=D \leq{ }_{h} C$ because $I \subseteq K$; thus $D$ is as required.

Case 2. For infinitely many $i<\omega$ we have $X_{i} \notin I$ and for every $i<\omega$ the algebra $A_{i} / I_{i}$ is finite.

If $X_{i} \notin I$, let $K_{i}$ be an extension of $I_{i}$ to a maximal principal ideal of $A_{i}$. Let $K$ be the ideal generated by $I \cup \bigcup K_{i}$. In this way $\bigoplus_{i<\omega}^{B} A_{i} / K$ is infinite (since, if $X_{i} \notin I$, then $\left.X_{i} \notin K\right)$ and $A_{i} / K_{i}=\left\{\emptyset, X_{i}\right\}$. Now note that it follows from definition 6.4 that for every $c \in \bigoplus_{i<\omega}^{B} A_{i}$ we have $\left[c\left(b, F,\left\{a_{i}: i \in F\right\}\right)\right]_{K}=\left[c\left(b^{\prime}, \emptyset, \emptyset\right)\right]_{K}$, where $b^{\prime}$ is defined by $b^{\prime}=b \cup\left\{i \in F: a_{i} \notin K_{i}\right\}$. Note that if $B^{\prime}=\{c(b, \emptyset, \emptyset): b \in B\}$, then there is an isomorphism $\pi: B \rightarrow B^{\prime} \leq \bigoplus^{B} A_{i}$, so $\left(\bigoplus_{i<\omega}^{B} A_{i}\right) / K=B^{\prime}\left(K \cap B^{\prime}\right)=$ $B /\left(\pi^{-1}\left(K \cap B^{\prime}\right)\right)=D \leq_{h} C$ because $I \subseteq K$; thus $D$ is as required.

Case 3. For some $i_{0}<\omega$ the algebra $A_{i_{0}} / I_{i_{0}}$ is infinite.

Let $K$ be generated by $I$ and $\bigcup_{i \neq i_{0}} X_{i}$. Note that it follows from definition 6.4 that for every $c \in \bigoplus_{i<\omega}^{B} A_{i}$ there are $a \in A_{i_{0}}$ such that $[c]_{K}=[a]_{K}$. Also note that $K_{i_{0}}=I_{i_{0}}$, so $\left(\bigoplus_{i<\omega}^{B} A_{i}\right) / K=A_{i_{0}} / I_{i_{0}}$. So $A_{i_{0}} / I_{i_{0}}=\left(\bigoplus_{i<\omega}^{B} A_{i}\right) / K \leq_{h}$ $\left(\bigoplus_{i<\omega}^{B} A_{i}\right) / I=C$, since $I \subseteq K$. So, $D=A_{i_{0}} / I_{i_{0}}$ works.

Lemma 6.6. Suppose $A_{i}$, for $i<\omega$, and $B$ are as in definition 6.4. Suppose that $X$ is an uncountable subset of the algebra $C=\bigoplus_{i<\omega}^{B} A_{i}$ of a regular cardinality $\kappa$. Then there are $Y \in[X]^{\kappa}$ and a finite $F \subseteq \omega$ such that $\langle Y\rangle_{C} \leq \bigoplus_{i \in F} A_{i} \oplus B$.

Proof. Let $\left(F_{\xi}: \xi<\kappa\right) \subseteq[\omega]^{<\omega},\left(b_{\xi}: \xi<\kappa\right) \subseteq B,\{a(\xi, i): \xi<\kappa, i<\omega\}$ be such that $X=\left\{c\left(b_{\xi}, F_{\xi},\left\{a(i, \xi): i \in F_{\xi}\right\}\right): \xi<\kappa\right\}$. Let $Y \subseteq X$ be a subset of $X$ of size $\kappa$ such that there is $F \in[\omega]^{<\omega}$ such that $F_{\xi}=F$ for each $\xi \in Y$. Let $B^{\prime}=\{c(b, \emptyset, \emptyset): b \cap F=\emptyset$ or $b \supseteq F\}$; then $B^{\prime}$ is isomorphic to a subalgebra of $B$ consisting of elements disjoint from or including $F$. Also $Y \subseteq \bigoplus_{i \in F}^{B} A_{i} \oplus B^{\prime} \subseteq$ $\bigoplus_{i \in F}^{B} A_{i} \oplus B$. Thus $F$ is as required.

The next definition and the following lemma concern subsets of $\omega_{1}$. We need to fix certain terminology. If $\alpha<\beta<\omega_{1}$, then $[\alpha, \beta)=\left\{\gamma<\omega_{1}: \alpha \leq \gamma<\beta\right\}$. If $X \subseteq \omega_{1}$, then $X^{\circ}=\left\{\alpha<\omega_{1}: \exists Y \subseteq X-\{\alpha\} \bigcup Y=\alpha\right\}$, i.e., $X^{\circ}$ is a collection of all limits of nonconstant sequences from $X$.

Definition 6.7. Let $\mathcal{C}=\left(C_{\alpha}: \alpha \in\left(\left(\omega_{1}\right)^{\circ}\right)^{\circ}\right)$ be a sequence such that $C_{\alpha}=$ $\left(c_{\alpha}^{n} ; n<\omega\right) \subseteq \alpha^{\circ}$ is strictly increasing and cofinal in $\alpha$ and $c_{\alpha}^{0}=0$ for any $\alpha \in$ $\left(\left(\omega_{1}\right)^{\circ}\right)^{\circ}$. Let $A, B$ be subalgebras of $\wp(\omega)$ such that $[\omega]^{<\omega} \subseteq B$. Then $\bigoplus_{\mathcal{C}}^{B} A$ is a subalgebra of the algebra of countable and cocountable subsets of $\omega_{1}$ which is generated by the elements of the union of an inductively constructed sequence of algebras $A_{\alpha} \subseteq \wp(\alpha)$ for $\alpha \in\left(\omega_{1}\right)^{\circ}$ satisfying $A_{\alpha}=A_{\beta} \oplus A^{\alpha}$ if $\alpha=\beta+\omega, \beta \in\left(\omega_{1}\right)^{\circ}$, and $A^{\alpha}$ is a copy of $A$ constructed on the set $[\beta, \alpha)$ and $A_{\alpha}=\bigoplus_{i<\omega}^{B} A_{i}$ where $A_{i}=\left\langle\left\{a \in \bigcup_{\beta<\alpha} A_{\beta} ; a \subseteq\left[c_{\alpha}^{i}, c_{\alpha}^{i+1}\right)\right\}\right\rangle$ if $\alpha \in\left(\left(\omega_{1}\right)^{\circ}\right)^{\circ}$.

Lemma 6.8. Suppose $\mathcal{C}, A, B$ are as in definition 6.7. Then

a) $\forall \alpha<\beta, \alpha, \beta \in\left(\omega_{1}\right)^{\circ}, A_{\alpha} \subseteq A_{\beta}$.

b) $\forall \alpha_{1}<\alpha_{2} \leq \beta$ with $\alpha_{1}, \alpha_{2}, \beta \in\left(\omega_{1}\right)^{\circ},\left[\alpha_{1}, \alpha_{2}\right) \in A_{\beta}$. 
Proof. The proof in by induction on $\beta<\omega_{1}$. If $\beta \in\left(\omega_{1}^{\circ}\right)-\left(\omega_{1}^{\circ}\right)^{\circ}$, then a) is clear by definition 6.7 and the inductive assumption. For b) consider two cases. The first case is when $\alpha_{2}<\beta$. Then $\left[\alpha_{1}, \alpha_{2}\right) \in A_{\alpha_{2}} \subseteq A_{\beta}$ by the inductive assumption b) and a). The second case, when $\alpha_{2}=\beta$, follows from the fact that $\left[0, \alpha_{1}\right) \in A_{\alpha_{1}} \subseteq A_{\beta}$, so $\left[\alpha_{1}, \alpha_{2}\right)=[0, \beta)-\left[0, \alpha_{1}\right) \in A_{\beta}$. Now consider the inductive step when $\beta \in\left(\omega_{1}^{\circ}\right)^{\circ}$. First prove b). Note that it is enough to prove that $\left[0, \alpha_{2}\right) \in A_{\beta}$. Since $[0, \beta)=\beta \in A_{\beta}$, we have to worry only about $\alpha_{2}<\beta$. Take $i_{0}<\omega$ minimal such that $c_{\beta}^{i_{0}}>\alpha_{2}$. Now note that by the inductive assumptions

$$
\left[c_{\beta}^{0}, c_{\beta}^{1}\right) \in A_{c_{\beta}^{1}}, \ldots,\left[c_{\beta}^{i-1}, c_{\beta}^{i}\right) \in A_{c_{\beta}^{i}}, \ldots,\left[c_{\beta}^{i_{0}-1}, \alpha_{2}\right) \in A_{c_{\beta}}^{i_{0}} .
$$

Thus $\left[c_{\beta}^{i-1}, c_{\beta}^{i}\right) \in A_{i}$ from definition 6.7 , so $\left[c_{\beta}^{i-1}, c_{\beta}^{i}\right) \cap\left[0, \alpha_{2}\right) \in A_{\beta}$ for $i \leq i_{0}$ by definition 6.7 , but $\bigcup_{i \leq i_{0}}\left(c_{\beta}^{i-1}, c_{\beta}^{i}\right]=c_{\beta}^{i_{0}}$, so $\left[0, \alpha_{2}\right) \in A_{\beta}$ as required. To conclude the proof of the lemma find the minimal $i_{0}<\omega$, such that $\alpha<c_{\beta}^{i}$, and note that by b) we may assume that for every $a \in A_{\alpha}$, for every $i \leq i_{0}$ we have $\left[c_{\beta}^{i-1}, c_{\beta}^{i}\right) \cap a \in A_{\alpha}$; thus $A_{\alpha} \subseteq A_{\beta}$ which was required.

Lemma 6.9. Suppose $\mathcal{C}, A, B$ are as in definition 6.7, and suppose that $D \leq_{h}$ $\bigoplus_{\mathcal{C}}^{B} A$. Then there are $E \leq D$ and $F \leq_{h} E$ such that $F \leq_{h} A$ or $F \leq_{h} B$.

Proof. Let $J$ be an ideal of $\bigoplus_{\mathcal{C}}^{B} A$, such that the obtained quotient $D$ is infinite. Let $a_{i} \in \bigoplus_{\mathcal{C}}^{B} A$ for $i<\omega$ be such that $\left[a_{i}\right]_{J} \neq\left[a_{j}\right]_{J}$ for all $i \neq j$. By definition 6.7 and lemma 6.8 , the algebra $\bigoplus_{\mathcal{C}}^{B} A$ is the union of an increasing sequence of algebras $A_{\alpha}$ for $\alpha<\omega_{1}$. So let $\alpha<\omega_{1}$ be such that $\left\{a_{i}: i<\omega\right\} \subseteq A_{\alpha}$. Now $E=A_{\alpha} /\left(A_{\alpha} \cap J\right)$ is infinite and $E \leq D$.

It is enough to prove that for every $\alpha<\omega_{1}$ and every $E \leq_{h} A_{\alpha}$, there is $F \leq_{h} E$ such that either $F \leq_{h} A$ or $F \leq_{h} B$.

The proof is by induction on $\alpha<\omega_{1}$. If $\alpha=\beta+\omega$, then by definition 6.7, $A_{\alpha}=A_{\beta} \oplus A$; thus by lemma 6.3 we have $E=A_{\beta}^{\prime} \oplus A^{\prime}$ where $A_{\beta}^{\prime} \leq_{h} A_{\beta}$ and $A^{\prime} \leq_{h} A$. Since $E$ is infinite, there is an infinite $E_{1} \leq_{h} E$ such that $E_{1}=A_{\beta}^{\prime} \leq A_{\beta}$ or $E_{1}=A^{\prime} \leq_{h} A$. In the latter case we are done. Otherwise by the inductive assumption about $A_{\beta}$ we get $E_{2} \leq_{h} E_{1}$ such that $E_{2} \leq_{h} A$ or $E_{2} \leq_{h} B$, thus $F=E_{2}$ works.

Now consider the case of $\alpha \in\left(\left(\omega_{1}\right)^{\circ}\right)^{\circ}$. First note that $A_{i}$ as in definition 6.7 is an infinite homomorphic image of $A_{c_{\alpha}^{i+1}}$. This follows from the fact that $\left[c_{\alpha}^{i}, c_{\alpha}^{i+1}\right) \in A_{c_{\alpha}^{i+1}}$ which follows from lemma $6.8 \mathrm{~b}$ ). Thus, if $E \leq_{h} A_{\alpha}=\bigoplus_{i<\omega}^{B} A_{i}$, then lemma 6.5 implies that there is $E_{1} \leq E$ such that $E_{1} \leq_{h} B$ or $E_{1} \leq_{h} A_{i}$ for some $i \in \omega$. In the first case we are done since $F=E_{1}$ works. In the second case we use the fact that $E_{1} \leq_{h} A_{i} \leq_{h} A_{c_{\alpha}^{i+1}}$ and the inductive assumption about $A_{c_{\alpha}^{i+1}}$ obtaining $F \leq_{h} E_{1}$ such that $F \leq_{h} B$ or $F \leq_{h} A$.

By $n A$ we will denote the sum $A \oplus \ldots \oplus A$ consisting of $n$ copies of algebra $A$.

Lemma 6.10. Suppose $A, B, \mathcal{C}$ are as in definition 6.7. Suppose that $X$ is a subset of the algebra $C=\bigoplus_{\mathcal{C}}^{B} A$ of a regular cardinality $\kappa$ bigger that $\aleph_{1}$. Then there are $n<\omega$ and $Y \in[X]^{\kappa}$ such that such that $\langle Y\rangle_{C} \leq n(A \oplus B)$.

Proof. By definition $6.7 \bigoplus_{\mathcal{C}}^{B} A$ is the union of an increasing chain of subalgebras $\left(A_{\alpha}: \alpha<\omega_{1}\right)$. Thus by the regularity of $\kappa$, there is $\alpha<\omega_{1}$ such that $X \cap A_{\alpha}$ is of the full size $\kappa$. So it is enough to prove, by induction on $\alpha<\omega_{1}$, that if $Y \subseteq A_{\alpha}$ is of size $\kappa$, then there are $n<\omega$ and $Z \in[Y]^{\kappa}$ such that $\langle Z\rangle_{A_{\alpha}} \leq n(A \oplus B)$. 
If $\alpha=\beta+\omega$ for some $\beta<\alpha$, then $A_{\alpha}=A_{\beta} \oplus A$, so $Y=\left\{v_{\xi} \cup a_{\xi}: \xi<\kappa\right.$, where $\left.v_{\xi} \in A_{\beta}, a_{\xi} \in A\right\}$. Consider $V=\left\{v_{\xi}: \xi<\kappa\right\}$ and $W=\left\{a_{\xi}: \xi<\kappa\right\}$. If $|V|<\kappa$, then there are $v \in A_{\beta}$ and $\Gamma \in[\kappa]^{\kappa}$ such that $v=v_{\xi}$ for $\xi \in \Gamma$ so $Z=\left\{v \cup a_{\xi}: \xi \in \Gamma\right\}$ is of size $\kappa$ and can be embedded into $A \oplus\{0,1\}$, so $\langle Z\rangle_{A_{\alpha}} \leq(A \oplus B)$ as required. If $|V|=\kappa$, apply the inductive assumption for $V$ and $A_{\beta}$ to conclude that there is $U \in[V]^{\kappa}$ such that $\langle U\rangle_{A_{\beta}} \leq n(A \oplus B)$ for some $n<\omega$; then for $Z=\left\{v_{\xi} \cup a_{\xi}: \xi \in U\right\}$ we have $\langle Z\rangle_{A_{\alpha}} \leq n(A \oplus B) \oplus A$ as $A_{\alpha}=A_{\beta} \oplus A$.

Now consider the case when $\alpha \in\left(\omega_{1}^{\circ}\right)^{\circ}$. Then $A_{\alpha}=\bigoplus_{i<\omega}^{B} A_{i}$, where $A_{i}$ are as in definition 6.7. By lemma 6.6 there are $U \in[Y]^{\kappa}$ and $F \in[\omega]^{<\omega}$ such that $\langle U\rangle_{A_{\alpha}} \leq \bigoplus_{i \in F} A_{i} \oplus B$ has size $\kappa$. Note that $A_{i} \leq A_{c_{\alpha}^{i+1}}$ by lemma $\left.6.8 \mathrm{~b}\right)$. For each $i \in F$ let $X_{i}=\left[c_{\alpha}^{i}, c_{\alpha}^{i+1}\right)$. Let $U_{i}=\left\{z \cap X_{i}: z \in\left[U \cap \bigoplus_{i \in F} A_{i} \oplus B\right]\right\}$. By regularity of $\kappa$ we may w.l.o.g. assume that either $\left|U_{i}\right|=1$ or $\left|U_{i}\right|=\kappa$. Let $G$ be the set of those $i \in F$ such that $U_{i}$ has size $\kappa$. Note that $G$ is nonempty. So we can apply successively the inductive assumption for $A_{i} \leq A_{c_{\alpha}^{i}}$ and $U_{i}$ for every $i \in G$. We obtain $Z_{i} \in\left[U_{i}\right]^{\kappa}$ and $n_{i}<\omega$ such that $\left\langle Z_{i}\right\rangle_{A_{i}} \leq n_{i}(A \oplus B)$ such that moreover

$$
\left\langle\left\{z_{i_{1}} \cup z_{i_{2}} \cup \ldots \cup z_{i_{k}}: z_{i} \in Z_{i}\right\}\right\rangle_{\oplus_{i \in G} A_{i}} \leq\left(\sum_{i \in G} n_{i}\right)(A \oplus B) .
$$

So now $Z=\left\{z_{i_{1}} \cup z_{i_{2}} \cup \ldots \cup z_{i_{k}} \cup X_{j_{1}} \cup \ldots \cup X_{j_{m}}: z_{i} \in Z_{i}, j \in F-G\right\}$ has this

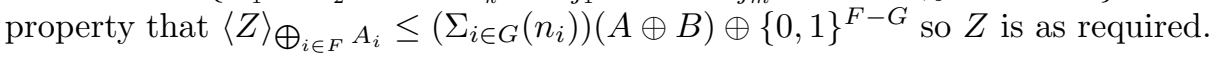

Theorem 6.11. Suppose that there exists a (hereditarily separable) Boolean algebra $A$ with its homomorphic cellularity relation equal to $\{(\omega, \lambda)\}$. Then there is an algebra (with its hereditary density $\omega_{1}$ ) whose homomorphic cellularity relation is $\left\{(\omega, \lambda),\left(\omega_{1}, \lambda\right)\right\}$.

Proof. By taking an infinite, homomorphic image we can w.l.o.g. assume that $[\omega]^{<\omega} \subseteq A \subseteq \wp(\omega)$. Now let $A=B$ and $\mathcal{C}$ be as in definition 6.7. We will prove that $\bigoplus_{\mathcal{C}}^{A} A$ satisfies the statement of the theorem. First we prove that every infinite homomorphic image of $A$ has size $\lambda$. By the assumption about $A$ and lemma 6.9 we get that $F$ as in lemma 6.9 has size $\lambda$; thus $D$ has to have size $\lambda$ for every homomorphic image $D$ of $\bigoplus_{\mathcal{C}}^{A} A$.

Now let us prove that homomorphic images of $\bigoplus_{\mathcal{C}}^{A} A$ may have cellularity either $\omega$ or $\omega_{1}$. $\bigoplus_{\mathcal{C}}^{A} A$ has cellularity $\omega_{1}$ itself, since $[\alpha, \beta) \in \bigoplus_{\mathcal{C}}^{A} A$ for every $\alpha<\beta<$ $\omega_{1}, \alpha, \beta \in(\omega)^{\circ}$, by lemma $\left.6.8 \mathrm{~b}\right)$ and a). Also $A$ is a homomorphic image of $\bigoplus_{\mathcal{C}}^{A} A$ (send $a \in \bigoplus_{\mathcal{C}}^{A} A$ to $a \cap \omega \in A$ ). So there are homomorphic images of $\bigoplus_{\mathcal{C}}^{A} A$ of cellularity $\omega$ or $\omega_{1}$. Now exclude other possibilities. Suppose the opposite; then there is $X$, a discrete family in $\bigoplus_{\mathcal{C}}^{A} A$ of size $\kappa>\omega_{1}$. ( $X$ is a left separated sequence if we assume countable hereditary density of $A$ and intend to prove that the hereditary density of $\bigoplus_{\mathcal{C}}^{A} A$ is $\omega_{1}$.) Now apply lemma 6.10 to get $[Y] \in[X]^{\kappa}$ such that for some $n\langle\omega\langle Y\rangle \leq n(A \oplus B)=2 n A$. This implies that $A$ includes a discrete (left-separated sequence) of size $|X|$ which is uncountable which contradicts the assumption about $A$ and the definition of direct sum.

\section{MA And a COUntably tight Algebra Without a POINT OF COUNTABLE CHARACTER}

In this section we construct a model of Martin's Axiom in which there is a minimally generated Boolean algebra with the properties as in the title of this section. 
Not all models of MA possess this property. For example models of PFA, the proper forcing axiom (see [Ba 2]), do not. It was proven by Alan Dow (see [Do]) that PFA implies that every countably tight Boolean algebra has a countably generated ultrafilter. On the other hand V. I. Malykhin (see [Mal]) constructed a model of $\mathrm{MA}(\sigma$-centered) (see $[\mathrm{W}])$ in which there is a countably tight Boolean algebra without an ultrafilter of countable character. I. Juhasz (in [Ju5]) has formulated a combinatorial principle which he calls $(t)$. This principle is implicitly present in Malykhin's proof and it implies the existence of the above algebra. Let us recall this principle which, on the other hand, is a weakening of the principle.

Definition 7.1. (t) is the following principle: There are a sequence $\left\{S_{\lambda}: \lambda \in\right.$ $\left.\operatorname{Lim}\left(\omega_{1}\right)\right\}$ and for each $\lambda \in \operatorname{Lim}\left(\omega_{1}\right)$ a disjoint partition $S_{\lambda}=\bigcup_{n \in \omega} S_{\lambda}^{n}$ such that $S_{\lambda}$ is cofinal $\omega$-type subset of $\lambda$ and for each $X \in\left[\omega_{1}\right]^{\omega_{1}}$ there is some $\lambda$ with $\left|X \cap S_{\lambda}^{n}\right|=\omega$ for all $n \in \omega$.

Proposition 7.2. $M A_{\omega_{1}}\left(\right.$ Precaliber $\left.\omega_{1}\right)$ implies $\neg(t)$

Proof. Consider the following partial order $P$ : The elements of $P$ are functions $f$ into 2 whose domains are at most countable subsets of $\omega_{1}$ having at most finitely many limit points in $\omega_{1}$ and such that the preimage $f^{-1}(\{1\})$ is finite. The order is inverse inclusion.

Let us prove that $P$ has precaliber $\omega_{1}$. Suppose $\left\{p_{\xi}: \xi<\omega_{1}\right\}$ is a sequence of conditions of $P$, and let $p_{\xi}^{-1}(\{1\})=X_{\xi}$. We may w.l.o.g. assume that $X_{\xi}$ 's form a $\Delta$-system with a root $X$, so that the sets $Y_{\xi}=X_{\xi}-X$ form an uncountable collection of finite pairwise disjoint subsets of $\omega_{1}$. By thinning out the sequence of conditions we may w.l.o.g. assume that

$$
\min Y_{\xi} \supseteq \sup \bigcup_{\eta<\xi} \operatorname{dom}\left(p_{\eta}\right) .
$$

Now, let us derive a contradiction from the assumption that $\left\{p_{\xi}: \xi<\omega_{1}\right\}$ contains no uncountable centered subfamily. It is easy to construct a sequence $\left\{A_{\alpha}: \alpha<\right.$ $\left.\omega^{2}+1\right\}$ of countable subsets of $P$ such that $A_{\alpha}$ is a maximal centered family of

$$
\left\{p_{\xi}: \xi>\sup \left\{\eta: p_{\eta} \in A_{\beta}, \beta<\alpha\right\}\right\} .
$$

Any $p_{\gamma} \in A_{\omega^{2}}$ is incompatible with some element $p_{\eta_{\beta}} \in A_{\beta}$ for every $\beta<\omega^{2}$; hence $\operatorname{dom}\left(p_{\gamma}\right) \cap Y_{\eta_{\beta}} \neq \emptyset$. But this implies that $\operatorname{dom}\left(p_{\gamma}\right)$ contains a subset of order type $\omega^{2}$ which implies that $\operatorname{dom}\left(p_{\gamma}\right)$ has infinitely many limit points, a contradiction.

Now suppose $\mathrm{MA}_{\omega_{1}}\left(\right.$ Precaliber $\left.\omega_{1}\right)$ holds and $\left(S_{\lambda}^{n}: n<\omega, \lambda \in \operatorname{Lim}\left(\omega_{1}\right)\right)$ witnesses $(t)$. Let $G$ be a generic subset of $P$ meeting the following dense subsets of $P$ :

$$
D_{\xi, \lambda, n}=\left\{p \in P: S_{\lambda}^{n}-p^{-1}(\{0\}) \text { is finite, } \sup \left(p^{-1}(\{1\})>\xi\right\}\right.
$$

for all $\xi \in \omega_{1}, \lambda \in \operatorname{Lim}\left(\omega_{1}\right), n \in \omega$. Then $X=\left\{\xi \in \omega_{1}: \exists p \in G p(\xi)=1\right\}$ contradicts the properties of the sequence $\left(S_{\lambda}^{n}: \lambda \in \operatorname{Lim}\left(\omega_{1}\right), n \in \omega\right)$ stated in $(t)$. This completes the proof of the proposition.

Now we turn to the construction of a model of MA in which there is a countably tight algebra without a point of countable character. One of the crucial arguments used to prove countable tightness is going to be based on fact 3.4. Thus we will be interested in the proofs of the countable chain condition for forcings $P(A, u)$. The actual proof will require not only the countable chain conditions of the forcings 
$P(A, u)$ but their finite products. Thus lemma 3.8 will find its use. The countable chain condition will be established based on the fact that if we add to an algebra $A$ an $(A, u)$-minimal element $g$ over a model containing a maximal antichain $X$ in $P(A, u, n)$ then $X$ stays a maximal antichain in the forcing

$$
P(\langle A \cup\{g\}\rangle,\langle u \cup\{g\}\rangle, n) .
$$

The following preservation lemma of slightly more complicated form will be required.

Lemma 7.3. Suppose $A \subseteq A_{0}$ are subalgebras of $\wp(\omega)$ and $F \subseteq u$ are a filter and an ultrafilter of $A$ respectively. Assume, moreover, that $u$ generates an ultrafilter in $A_{0}$. Suppose that $X \subseteq\left(F^{+}\right)^{n}$ is a maximal antichain in the forcing $P\left(A_{0}, F, n\right)$. Suppose $g$ is $(A, u)$-minimal over $\mathcal{M} \ni A, A_{0}, u, F, X$ and that $\mathcal{M} \cap u$ generates $u$. Then $X$ is a maximal antichain in $P\left(A_{0}\langle g\rangle,\langle F\rangle_{A_{0}\langle g\rangle}^{F i}, n\right)$.

Proof. Let $p \in P(A, u)$ and $p \|-r \in P\left(A_{0}\langle g\rangle, F, n\right)$. We may w.l.o.g. assume that there are elements $e_{i}, f_{i}$ of $A_{0}$ for $i \leq n$ such that

$$
p \Vdash r=\left(g \cap e_{1} \cup g^{-1} \cap f_{1}, \ldots, g \cap e_{n} \cup g^{-1} \cap f_{n}\right) .
$$

We claim that there are at most one $i \leq n$ and at most one $j \leq n$ such that $e_{i} \in\langle u\rangle, f_{j} \in\langle u\rangle$ where $\langle u\rangle$ is the ultrafilter generated in $A_{0}$ by $u$. This follows from the fact that we require the coordinates of our conditions to be disjoint. Suppose for example that $e_{1}, e_{2} \in\langle u\rangle$; then $g \cap e_{1} \cap e_{2} \neq \emptyset$ by proposition 2.2 and fact 3.2, which contradicts the disjointness of the coordinates of conditions in $P(A, F, n)$. Also consider the element $g \cap e_{i} \cup g^{-1} \cap f_{i}$ for $e_{i}, f_{i} \notin\langle u\rangle$. Then $g \cap e_{i}, g \cap f_{i} \in A_{0}$, since $g, g^{-1}$ are minimal for $\left(A_{0}, u\right)$. Also note that if $e \in\langle u\rangle$, then $e \cap g^{k}=e^{\prime} \cap g^{k} \cup h$, where $e^{\prime} \in u$ and $h \in A_{0}-\langle u\rangle, k=-1,1$, because $u$ generates an ultrafilter in $A_{0}$. And finally note that we can w.l.o.g. assume that $i \neq j$; indeed, $g^{-1} \cap e_{i} \cup g \cap f_{i}=\left(g^{-1} \cap e_{i}-f_{i}\right) \cup\left(g \cap f_{i}-e_{i}\right) \cup\left(e_{i} \cap f_{i}\right) \in A_{0}$ since $e_{i}-f_{i}, f_{i}-e_{i} \notin\langle u\rangle$.

For simplicity let us assume that $\{i, j\} \subseteq\{1,2\}$. By the above discussion we may w.l.o.g. assume that $p$ forces that $r$ is of one of the following forms, for $d_{1}, d_{2}, d_{3}, \ldots, d_{n} \in A_{0}-\langle u\rangle$ and $e_{1}, e_{2}, f_{1}, f_{2} \in A$ :

$$
\begin{aligned}
& \text { *) } \\
& r=\left(\left(g \cap e_{1}\right) \cup d_{1},\left(g^{-1} \cap f_{2}\right) \cup d_{2}, d_{3}, \ldots, d_{n}\right) . \\
& \text { **) } \\
& r=\left(\left(g^{-1} \cap f_{1}\right) \cup d_{1},\left(g \cap e_{2}\right) \cup d_{2}, d_{3}, \ldots, d_{n}\right) .
\end{aligned}
$$

Let us consider the following conditions respectively:

$$
\begin{aligned}
& p^{*}=\left(\left(p_{1} \cap e_{1}\right) \cup d_{1},\left(p_{-1} \cap f_{2}\right) \cup d_{2}, d_{3}, \ldots, d_{n}\right) . \\
& p^{*}=\left(\left(p_{-1} \cap f_{1}\right) \cup d_{1},\left(p_{1} \cap e_{2}\right) \cup d_{2}, d_{3}, \ldots, d_{n}\right) .
\end{aligned}
$$

First note that in both cases $p^{*} \in P\left(A_{0}, F, n\right)$. All the coordinates must be disjoint since $p \| p_{i}^{*} \subseteq r_{i}$ for $i \leq n$ and $p \| r \in P\left(A_{0}, F, n\right)$. Also note that $p \Vdash p_{i}^{*} \subseteq r_{i} \notin F$.

Now find $x \in X$ such that $x \| p^{*}$, using the fact that $X$ is a maximal antichain in $P\left(A_{0}, F, n\right)$. Consider the following $q$ respectively:

$$
\begin{aligned}
& q=\left(p_{-1} \cup\left[e_{1} \cap \bigcup_{3 \leq i \leq n} x_{i}\right], p_{1} \cup\left[f_{2} \cap \bigcup_{3 \leq i \leq n} x_{i}\right]\right) . \\
& q=\left(p_{-1} \cup\left[e_{2} \cap \bigcup_{3 \leq i \leq n} x_{i}\right], p_{1} \cup\left[f_{1} \cap \bigcup_{3 \leq i \leq n} x_{i}\right]\right) .
\end{aligned}
$$


We claim that $q \in P(A, u)$. As $\bigcup_{3 \leq i \leq n} x_{i} \in F^{+}$, by the assumption about $X$, both coordinates are not in $\langle u\rangle$; also $f_{i}, e_{i}, p_{i}, x_{i} \in A$, because $F \subseteq A$. For disjointness of the coordinates we have to check, respectively, that

$$
\begin{aligned}
& p_{1} \cap\left[e_{1} \cap \bigcup_{3 \leq i \leq n} x_{i}\right]=p_{-1} \cap\left[f_{2} \cap \bigcup_{3 \leq i \leq n} x_{i}\right]=\emptyset . \\
& p_{-1} \cap\left[f_{1} \cap \bigcup_{3 \leq i \leq n} x_{i}\right]=p_{1} \cap\left[e_{2} \cap \bigcup_{3 \leq i \leq n} x_{i}\right]=\emptyset .
\end{aligned}
$$

But the above follows from the fact that $x$ is compatible with $p^{*}$, so $p \geq q \in P(A, u)$. Now we claim that $q \|-r$ is compatible with $x$. For this we only need to check, respectively, that

$$
\begin{aligned}
& q \Vdash\left(g \cap e_{1}\right) \cap \bigcup_{3 \leq i \leq n} x_{i}=\left(g^{-1} \cap f_{2}\right) \cap \bigcup_{3 \leq i \leq n} x_{i}=\emptyset . \\
& q \Vdash\left(g^{-1} \cap f_{1}\right) \cap \bigcup_{3 \leq i \leq n} x_{i}=\left(g \cap e_{2}\right) \cap \bigcup_{3 \leq i \leq n} x_{i}=\emptyset .
\end{aligned}
$$

This does not cause any problems since we have, respectively,

$$
\begin{aligned}
& {\left[e_{1} \cap \bigcup_{3 \leq i \leq n} x_{i}\right] \subseteq q_{-1},\left[f_{2} \cap \bigcup_{3 \leq i \leq n} x_{i}\right] \subseteq q_{1} .} \\
& {\left[f_{1} \cap \bigcup_{3 \leq i \leq n} x_{i}\right] \subseteq q_{1},\left[e_{2} \cap \bigcup_{3 \leq i \leq n} x_{i}\right]=\subseteq q_{-1} .}
\end{aligned}
$$

And obviously $q \Vdash q_{-1} \subseteq g^{-1}, q_{1} \subseteq g$. So the proof of the lemma is completed.

Lemma 7.4. Suppose that $n, k<\omega$ and $A_{1}, \ldots, A_{k}$ are Boolean algebras and for all $i \leq k, F_{i}$ is a filter in $A_{i}$. Suppose that $X$ is a maximal antichain in the product $P\left(A_{1}, F_{1}, n\right) \times \ldots \times P\left(A_{k}, F_{k}, n\right)$. Suppose that for every ultrafilter $u$ of $A_{i}$ for $i \leq k$ we have assigned an algebra $A_{u} \subseteq A_{i}$ such that $A_{u} \cap u$ generates $u$.

Let $U_{i}=\left\{u: F_{i} \subseteq u \in U l t\left(A_{i}\right): u\right.$ is $\omega$-generated in $\left.A_{i}\right\}$ and

$$
P=\prod_{u \in U_{1}} P\left(A_{u}, u\right) \times \ldots \times \prod_{u \in U_{k}} P\left(A_{u}, u\right)
$$

(the products are taken with finite supports).

Then in the generic extension $V^{P}, X$ is a maximal antichain in the forcing

$$
P\left(B_{1},\left\langle F_{1}\right\rangle^{F i}, n\right) \times \ldots \times P\left(B_{k},\left\langle F_{k}\right\rangle^{F i}, n\right)
$$

where for $i \leq k$

$$
B_{i}=\left\langle A_{i} \cup\left\{a_{G(u)}: u \in U_{i}\right\}\right\rangle
$$

where $G$ is the generic object in $P$ such that $V^{P}=V[G]$.

Proof. We will need the following elementary

Fact. Suppose that $P_{1}, \ldots, P_{m}, P_{m+1}$ are forcing notions for some $m<\omega$ and $X \subseteq$ $P_{1} \times \ldots \times P_{m} \times P_{m+1}$. Then the following are equivalent:

a) $X$ is a maximal antichain in $P_{1} \times \ldots \times P_{m} \times P_{m+1}$. 
b) For every $p=\left(p_{1}, \ldots, p_{m}\right) \in P_{1} \times \ldots \times P_{m}$ the set

$X(p)=\left\{x: \exists\left(x_{1}, \ldots, x_{m}\right)\right.$ compatible with $\left(p_{1}, \ldots, p_{m}\right)$ such that $\left.\left(x_{1}, \ldots, x_{m}, x\right) \in X\right\}$

is a maximal antichain in $P_{m+1}$.

By induction on $l \leq k$, we prove that in $V^{P}, X$ is a maximal antichain in $P\left(B_{1},\left\langle F_{1}\right\rangle^{F i}, n\right) \times \ldots \times P\left(B_{l},\left\langle F_{l}\right\rangle^{F i}, n\right) \times P\left(A_{l+1}, F_{l+1}, n\right) \times \ldots \times P\left(A_{k}, F_{k}, n\right)$.

Suppose that we are done till $l<k$. Let $\left(u_{\xi}: \xi<\alpha\right)$ be an enumeration of distinct countably generated ultrafilters of $A_{l+1}$ in $V$. Define

$$
P_{l+1}^{\eta}=\prod_{u \in U_{1}} P\left(A_{u}, u\right) \times \ldots \times \prod_{u \in U_{l-1}} P\left(A_{u}, u\right) \times \prod\left\{P\left(A_{u_{\xi}}, u_{\xi}\right): \xi<\eta\right\}
$$

and define

$$
B_{l+1}^{\eta}=\left\langle A_{l+1} \cup\left\{a_{G\left(u_{\xi}\right)}: \xi<\eta\right\}\right\rangle .
$$

So it is enough to prove, by induction on $\eta<\alpha$, that in $V^{P_{l+1}^{\eta}}, X$ is maximal antichain in

$P\left(B_{1},\left\langle F_{i}\right\rangle^{F i}, n\right) \times \ldots \times P\left(B_{l},\left\langle F_{l}\right\rangle^{F i}, n\right) \times P\left(B_{l+1}^{\eta},\left\langle F_{l+1}\right\rangle^{F i}, n\right) \times \ldots \times P\left(A_{k}, F_{k}, n\right)$.

Note that each condition $p^{l+1}=\left(p_{1}, \ldots, p_{l}, p_{l+2}, \ldots, p_{k}\right)$ of the forcing $P\left(B_{1},\left\langle F_{i}\right\rangle^{F i}, n\right)$ $\times \ldots \times P\left(B_{l},\left\langle F_{l}\right\rangle^{F i}, n\right) \times P\left(A_{l+2}, F_{l+2}, n\right) \times \ldots \times P\left(A_{k}, F_{k}, n\right)$ is already in the model $V^{P_{l+1}^{0}}$; thus $X\left(p^{l+1}\right)$ as defined in the fact is in the same model. So it is enough to show by induction on $\eta<\alpha$ that in $V^{P_{l+1}^{\eta}}, X\left(p^{l+1}\right)$ is a maximal antichain in $P\left(B_{l+1}^{\eta},\left\langle F_{l+1}\right\rangle^{F i}, n\right)$. By the inductive assumption it is true for $\eta=0$. Suppose we are done for $\xi<\eta$. If $\eta$ is a limit ordinal, there is nothing to prove as the forcings involved are increasing unions of preceding forcings in which $X\left(p^{l+1}\right)$ was a maximal antichain by the inductive assumption. So, suppose that $\eta=\xi+1$ for some ordinal $\xi$. We are going to use Lemma 7.3, for $A=A_{u_{\eta}}, A_{0}=B_{l+1}^{\xi}, F=$ $F_{l+1}, u=u_{\eta}, \mathcal{M}=V^{P_{l+1}^{\xi}}$. Now we check that the assumptions of lemma 7.3 are satisfied. By the definitions of the involved objects $u_{\eta} \cap A_{\eta}$ is an ultrafilter of $A_{u_{\eta}}$ which extends $F_{l+1}$ and generates an ultrafilter in $A_{l+1}$. We have to note that $u_{\eta} \cap A_{\eta}$ generates an ultrafilter in $A_{0}=B_{l+1}^{\xi}$; indeed $B_{l+1}^{\xi}$ was obtained from $A_{l+1}$ by adding $\left(A_{l+1}, u_{\delta}\right)$-minimal elements for $u_{\delta} \neq u_{\eta}$. Thus $u_{\eta} \cap A_{\eta}$ still generates an ultrafilter in $B_{l+1}^{\xi}$ by definition 2.1 and fact 3.2. (our ultrafilters are countably generated and so they are generated from every model that contains them). The next assumption of lemma 7.3 that we have to check is that $X\left(p^{l+1}\right)$ is a maximal antichain in $P\left(B_{l+1}^{\xi},\left\langle F_{l+1}\right\rangle^{F i}, n\right)$ but this is our inductive assumption. Now by the product lemma, (see $[\mathrm{K}])$ the element $a_{G\left(u_{\eta}\right)}$ is $\left(A_{u_{\eta}}, u_{\eta}\right)$-minimal over $V^{P_{l+1}^{\xi}}$ and $X\left(p^{l+1}\right), A_{u_{\eta}}, B_{l+1}^{\xi}, u_{\eta}, F_{l+1}$ all are in $\mathcal{M}=V^{P_{l+1}^{\xi}}$. So the use of lemma 7.3 is legitimate, and hence $X\left(p^{l+1}\right)$ is still a maximal antichain in $P\left(B_{l+1}^{\eta}\right)$, and this completes the inductive step and the proof of the lemma.

Before entering the proof of the main theorem of this section we have to recall certain results from [De]. Those results will enable us to obtain a model of MA without adding uncountable branches to trees of height $\omega_{1}$. This will increase our control over new uncountably generated ultrafilters in $T$-algebras, as those ultrafilters correspond to uncountable branches of $T$ by lemma 2.8. K. Devlin has 
proven in [De] (Corollary 3.15) that if $B$ is a complete Boolean algebra satisfying the c.c.c., then the following are equivalent:

(i) There are an $\omega_{1}$-tree $T$ and $a B$-name $\dot{b}$ such that $B \Vdash$ " $\dot{b}$ is an uncountable branch in $T$ which is not in $V$ ".

(ii) There is a subset $U \subseteq B$ such that $\left\langle U, \leq_{B}\right\rangle$ is a Souslin tree and for each $\alpha<\omega_{1}$, the $\alpha$-the level of $U$ is a maximal antichain in $B$.

We will call a c.c.c. forcing notion $P$ a Souslin free notion of forcing if and only if its Boolean completion (see $[\mathrm{K}]$ ) does not satisfy either of the above equivalent conditions. Since the generic extension obtained by a forcing notion and by its Boolean completion are the same, no Souslin free forcing notion introduces new branches to $\omega_{1}$-trees from the ground model. Note that actually we may say the same about any tree of height $\omega_{1}$ not necessarily having countable levels. It follows from the fact that a c.c.c. notion of forcing introduces an uncountable branch in a tree of height $\omega_{1}$ if and only if it introduces a new uncountable branch in an $\omega_{1}$-tree. This follows from the fact that if $\dot{b}$ is a name for an uncountable branch in a tree $T$, then $S=\{t \in T: \exists p \in P p \|-t \in b\}$ is a subtree of $T$ with countable levels (since $P$ satisfies the c.c.c) and $P \| \dot{b} \subseteq S$.

Note that if $P$ is not a Souslin free forcing notion, then we can consider the standard forcing $Q$ with finite conditions which specializes the Souslin tree $U$ in the Boolean completion of $P$ ( see [BMR] or [Ba1]). Note that $Q$ forces that $U$ does not satisfy the c.c.c., and so as $P$ is dense in its Boolean completion, $Q$ forces that $P$ does no longer satisfy the c.c.c. As $Q^{2}$ satisfies the c.c.c. (see and generalize the proof in [Ba1]), $Q$ is a Souslin free forcing. Thus one can add MA by an iteration $\left(P_{\alpha}, P^{\alpha}\right)_{\alpha<\kappa^{+}}$such that for each $\alpha<\kappa^{+}$, we have

$$
P_{\alpha} \Vdash P^{\alpha} \text { is a Souslin free forcing notion }
$$

because, if a forcing with which we need to force in some intermediate stage in order to ensure MA in the final model does not satisfy the c.c.c., we can force at this stage by a Souslin free forcing which kills the c.c.c. of the original forcings instead. So for all c.c.c. forcings and for all families of $\kappa$ many of their dense sets in the final model we have an appropriate generic object. To conclude this discussion of the results from [De], note that if a finite support iteration $\left(\left(P_{\alpha}, P^{\alpha}\right): \alpha<\lambda+1\right)$ for a limit $\lambda$ does introduce a new branch to an $\omega_{1}$-tree, then there is $\nu<\lambda$ so that the forcing $P_{\nu}$ already introduces a new uncountable branch to the tree (Lemma 3.7 of $[\mathrm{De}])$. Thus finite support iterations satisfying $*$ ) are Souslin free themselves. We will call those iterations iterations of Souslin free forcing notions.

Theorem 7.5. Suppose that ZFC is a consistent theory. Then so is the following theory for $\kappa$ an arbitrary cardinal: " $Z F C+M A_{\kappa}+$ There is a Boolean algebra $A$ whose every ultrafilter has uncountable character and for every ultrafilter $u$ of $A$ there is a subalgebra $A_{u}$ of $A$, such that the forcing notion $P\left(A_{u}, u \cap A_{u}\right)$ satisfies the c.c.c. and $u$ is generated from $A_{u} \cap u$. In particular $A$ has countable tightness".

Proof. The proof uses the method of iterated forcing. Let us fix cardinal $\kappa$. By induction on $\alpha<\kappa^{+}$we define:

1) A finite support iteration of forcings $P_{\alpha}$ of length $\alpha$ such that $P_{\alpha} \mid \beta=P_{\beta}$ for each $\beta<\alpha$.

2) A $P_{\alpha}$-name for an acceptable tree $\dot{T}_{\alpha}$ such that $\dot{T}_{\beta} \prec \dot{T}_{\alpha}$ for each $\beta<\alpha$.

3) A $P_{\alpha}$ name $\dot{A}_{\alpha}=\left\langle\dot{a}_{t}: t \in \dot{T}_{\alpha}\right\rangle$ for a $\dot{T}_{\alpha}$-algebra such that $\dot{A}_{\beta} \prec \dot{A}_{\alpha}$ for each $\beta<\alpha$. 
Suppose that we are given all the above objects for $\beta<\alpha$. We define $P_{\alpha}, \dot{T}_{\alpha}, \dot{A}_{\alpha}$ as follows:

Case 1. $\alpha$ is a limit ordinal.

1) Then $P_{\alpha}$ is a direct limit of $\left(P_{\beta},: \beta<\alpha\right)$, i.e., $P_{\alpha}=\bigcup_{\beta<\alpha} P_{\beta}$.

2) $\dot{T}_{\alpha}$ is a $P_{\alpha}$-name for $\bigcup_{\beta<\alpha} \dot{T}_{\beta}$.

3) $\dot{A}_{\alpha}$ is a $P_{\alpha}$-name for $\bigcup_{\beta<\alpha} \dot{A}_{\beta}$.

Case 2. $\alpha=\beta+1$ for some $\beta<\kappa^{+}$.

The following definition depends on $\dot{Q}_{\beta}$ which will be specified at the end of the definition, depending on subcases:

1) $P_{\alpha}=P_{\beta} *\left(\left(\dot{P}^{\beta}\right)^{\prime} * \dot{Q}^{\beta}\right)$, where $\left(\dot{P}^{\beta}\right)^{\prime}$ is a name for a product with finite supports of countably many copies of the forcing $\dot{P}^{\beta}$ which is also a finite support product such that

$$
P_{\beta} \Vdash \dot{P}^{\beta}=\prod\left\{P\left(A_{\dot{b}}, u_{\dot{b}}\right): \dot{b} \text { is a maximal branch of } \dot{T}_{\beta}\right\} \text {. }
$$

(We are using the terminology of lemma 2.8.)

2) $P_{\alpha} \Vdash \dot{T}_{\alpha}=\dot{T}_{\beta} \cup\left\{\dot{b} \frown i: \dot{b}\right.$ is a maximal, countable branch of $\left.\dot{T}_{\beta}\right\}$.

3) $P_{\alpha} \Vdash \dot{A}_{\alpha}=\left\langle\dot{A}_{\beta} \cup\left\{a_{\dot{b}-i}: \dot{b}\right.\right.$ is a maximal countable branch of $\left.\left.\dot{T}_{\beta}\right\}\right\rangle$,

where $a_{\dot{b} \frown i}=\bigcup\left\{p_{1}: \exists p_{-1}\left(p_{-1}, p_{1}\right) \in \dot{G}_{P^{\beta}}(\dot{b})\right\}$, where $\dot{G}_{P^{\beta}}$ is the canonical name for the $\dot{b}$-th coordinate of the generic object in the product $P^{\beta}$.

Now, as we have promised, we specify the forcing $\dot{Q}^{\beta}$. It depends whether $\operatorname{cf}(\beta)=\omega_{1}$ or not. If the cofinality is $\omega_{1}$, then $\dot{Q}^{\beta}$ is a name for the trivial forcing. Otherwise we choose the name $\dot{Q}^{\beta}$ so that $P^{\beta} *\left(\dot{P}^{\beta}\right)^{\prime}$ forces that it is the name for the next forcing $\dot{R}^{\beta}$, with which we need to force to ensure MA in the final model if $P_{\beta} *\left(\dot{P}^{\beta}\right)^{\prime} \Vdash \dot{R}^{\beta}$ is Souslin free, or otherwise $P_{\beta} *\left(\dot{P}^{\beta}\right)^{\prime} \Vdash \dot{Q}^{\beta}$ is a Souslin free forcing such that $\dot{Q}^{\beta} \|-\dot{R}^{\beta}$ is not c.c.c. This completes the description of the construction. The main part of the proof of the theorem is a simultaneous proof by induction on $\alpha<\kappa^{+}$of the following two claims.

Claim 1. $P_{\alpha}$ is Souslin free and satisfies the c.c.c.

Claim 2. $c f(\alpha) \neq \omega_{1}$ or one of the following holds in $V^{P_{\alpha}}$ : for any uncountable maximal branch $b$ of $T_{\alpha}$

a) There is $\beta<\alpha$ such that $b \subseteq T_{\beta}$ and $b \in V^{P_{\beta}}$.

b) There is a continuous, nondecreasing function $f_{b}: \omega_{1} \rightarrow \alpha$ with the range cofinal in $\alpha$ such that for each $\xi \in \omega_{1}$ we have

$$
b\left|\xi \in V^{P_{f_{b}(\xi)}} \& b\right| \xi \text { is a maximal branch of } T_{f_{b}(\xi)} .
$$

Proof of the claims. First consider the case when $\alpha$ is a limit ordinal. Claim 1 is then trivial as iterations of c.c.c. Souslin free forcing are again c.c.c., Souslin free (see [De]). Claim 2 says something only in the case when $\operatorname{cf}(\alpha)=\omega_{1}$. So we may w.l.o.g. assume that $c f(\alpha)=\omega_{1}$. Now, note that we may w.l.o.g. assume that there is no $\beta<\alpha$ such that $b \subseteq T_{\beta}$, as in this case $b$ has to be an element of $V^{P_{\beta}}$ : in $V^{P_{\beta}}$ the forcing $P_{[\beta, \alpha)}$ is Souslin free by the inductive assumption and results from [De]; hence the latter forcing does not add any new uncountable branches of $T_{\beta}$. It follows that the first part of the alternative in claim 2 holds, unless we assume that there is no $\beta<\alpha$ such that $b \subseteq T_{\beta}$. We aim at proving that the second part of the alternative in claim 2 holds. 
First go back to $V$ and find a continuous, nondecreasing function $g_{b}: \omega_{1} \rightarrow \alpha$ with the range unbounded in $\alpha$ such that in $V^{P_{\alpha}}$ we have

$$
\forall \xi \in \omega_{1} b \mid \xi \in V^{P_{g_{b}(\xi)}} .
$$

As $b$ is really a function from $\omega_{1}$ into $\{-1,1\}$ and $P_{\alpha}$ is an iteration of c.c.c. forcings, this task can be easily accomplished.

Work again in $V^{P_{\alpha}}$. Since $\left(T_{\xi}: \xi<\alpha\right)$ is a continuous, increasing sequence of trees which end-extend the previous elements of the sequence, there is a continuous, nondecreasing function $h_{b}: \omega_{1} \rightarrow \alpha$ such that

$$
\forall \xi \in \omega_{1} b \cap T_{h_{b}(\xi)}=b \mid \xi .
$$

Since we assumed that for no $\beta<\alpha$ do we have $b \subseteq T_{\beta}$, we conclude that the range of $h_{b}$ is unbounded in $\alpha$. So we can find a club subset $C$ of $\omega_{1}$ such that for all $\xi \in C$, we have $g_{b}(\xi)=h_{b}(\xi)$. Now, if we compose, say, $h_{b}$ with the function enumerating elements of $C$, we obtain a function $f_{b}: \omega_{1} \rightarrow \alpha$, which is continuous, nondecreasing and has its range unbounded in $\alpha$ and satisfies the condition

$$
\forall \xi \in \omega_{1} b\left|\xi \in V^{P_{f_{b}(\xi)}} \& b\right| \xi=b \cap T_{f_{b}(\xi)} .
$$

We claim that $f_{b}$ satisfies the second part of the alternative of Claim 2. This does not follow immediately from the fact that $T_{\xi} \prec T_{\alpha}$ and $b$ is a maximal branch of $T_{\alpha}$, since our trees are not normal. Nevertheless the fact that $T_{\xi}$ is acceptable is sufficient. Namely, if $b \cap T_{f_{b}(\xi)}$ is not a maximal branch, then there is an $i \in\{-1,1\}$ such that $b \frown i$ is in $T_{f_{b}(\xi)}$, but then $b \frown(-i)$ must be in the tree, but one of them has to be in $b$ which gives a contradiction. Thus, the proof of the claims in the limit case is completed.

Let us move to the proof of the claims in the case when $\alpha$ is a successor, say $\alpha=\beta+1$. Let us begin with the crucial subcase when $\operatorname{cf}(\beta)=\omega_{1}$. Let us begin this subcase with the proof of Claim 1. If we prove that in $V^{P_{\beta}}$ the forcing $P^{\beta}$ is Souslin free and c.c.c., we will be done with Claim 1, since an iteration of two Souslin free forcings is Souslin free itself by results of [De]. So, let us aim at this, by proving first that establishing the following subclaim will be sufficient.

Subclaim. Suppose that $n, k<\omega$. In $V^{P_{\beta}}$ if $b_{1}, \ldots, b_{k}$ are distinct uncountable branches of $T_{\beta}$, then

$$
P\left(A_{b_{1}}, u_{b_{1}}, n\right) \times \ldots \times P\left(A_{b_{k}}, u_{b_{k}}, n\right)
$$

satisfies the c.c.c.

Now we present the rest of the proof of Claim 1 , in the case when $\alpha=\beta+$ $1, \operatorname{cf}(\beta)=\omega_{1}$, assuming the subclaim. Work in $V_{\beta} \cdot\left(P^{\beta}\right)^{\prime}$ is a product with finite supports of countably many copies of certain forcings. Thus if it satisfies the c.c.c., then its square satisfies the c.c.c.; thus by results from [De], it is Souslin free if it is c.c.c. Now note that $P^{\beta}$ is also a product with finite supports, so to prove that such a countable product of such forcings satisfies the c.c.c. it is enough to prove that its every finite subproduct satisfies the c.c.c. (see $[K]$ ). But every finite subproduct of $P^{\beta}$ is included in a product of the form

$$
P^{n}\left(A_{b_{1}}, u_{b_{1}}\right) \times \ldots \times P^{n}\left(A_{b_{k}}, u_{k}\right)
$$

for some $n, k<\omega$ and distinct maximal branches of $T_{\beta}$. By lemma 3.8 the countable chain condition in a product as above is implied by the countable chain condition 
in the product

$$
P\left(A_{b_{1}}, u_{b_{1}}, 2^{n}\right) \times \ldots \times P\left(A_{b_{k}}, u_{b_{k}}, 2^{n}\right) .
$$

This completes the proof of Claim 1 in our case of $\alpha=\beta+1, \operatorname{cf}(\beta)=\omega_{1}$ subject to the Subclaim.

Proof of the subclaim. We work in $V^{P_{\beta}}$. We are going to use the inductive assumption for $\beta$. We want to prove that by Claim 2 for $\beta$ we can assume without loss of generality that the branches $b_{1}, \ldots, b_{k}$ in the statement of the subclaim can be assumed to satisfy the second part of the alternative in Claim 2. Since Claim 2 holds in $V^{P_{\beta}}$, it is enough to prove that the factors whose branches satisfy the first part of the alternative from Claim 2 do not affect the chain condition in the product from the statement of the subclaim. For this it is enough to note that they are $\sigma$-centered. Indeed if $b \in V^{P_{\gamma}}$ and $b \subseteq T_{\gamma}$ for some $\gamma<\beta$, then at the stage $\gamma+1$ we force with the product with finite supports of countably many copies of the forcing $P\left(A_{b}, u_{b}\right)$. Thus in $V^{P_{\gamma+1}}$, this forcing becomes a $\sigma$-centered forcing; thus it is a forcing of that kind in $V^{P_{\beta}}$.

Now suppose that the subclaim is false, that is that there is a uncountable antichain $X$ included in the product from the subclaim. We may w.l.o.g. assume that $X$ is a maximal antichain. Since for each $\xi<\omega_{1}$ the forcing $P\left(A_{b \mid \xi}, u_{b \mid \xi}\right)$ is countable (where $u_{b \mid \xi}=\left\{u_{t}: t \in b, \operatorname{dom}(t) \subseteq \xi\right\}, A_{b \mid \xi}=\left\langle u_{b \mid \xi}\right\rangle$ ) and since $X$ is a member of $V^{P_{\beta}}$ we can find a nondecreasing, continuous function $f: \omega_{1} \rightarrow \beta$ with an unbounded range in $\beta$ such that for each $\xi \in \omega_{1}$ we have for $X \mid \xi=$ $X \cap\left[P\left(A_{b_{1} \mid \xi}, u_{b_{1} \mid \xi}, n\right) \times \ldots \times P\left(A_{b_{k} \mid \xi}, u_{b_{k} \mid \xi}, n\right)\right]$ the following condition:

$X\left|\xi \in V^{P_{f(\xi)}} \& X\right| \xi$ is a maximal antichain

$$
\text { in } P\left(A_{b_{1} \mid \xi}, u_{b_{1} \mid \xi}, n\right) \times \ldots \times P\left(A_{b_{k} \mid \xi}, u_{b_{k} \mid \xi}, n\right) .
$$

Now, by claim 2 in $V^{P_{\beta}}$, by the second part of its alternative we are given functions $f_{b_{1}}, \ldots, f_{b_{k}}$ (recall that we have proven that we can restrict ourselves to branches satisfying the second part of the alternative). By continuity and unboundedness we can find $\xi<\omega_{1}$ such that $f_{b_{1}}(\xi)=\ldots=f_{b_{k}}(\xi)=f(\xi)=\theta<\beta$ and such that $b_{i}\left|\xi \neq b_{j}\right| \xi$ for all $i<j \leq k$.

Now by induction on $\eta$ where $\theta \leq \eta<\beta$, we will prove that in $V^{P_{\eta}}$

!) $X \mid \xi$ is a maximal antichain in $P\left(B_{1}^{\eta},\left\langle u_{b_{1} \mid \xi}\right\rangle^{F i}, n\right) \times \ldots \times P\left(B_{k}^{\eta},\left\langle u_{b_{k} \mid \xi}\right\rangle^{F i}, n\right)$

where

$$
B_{i}^{\eta}=\left\langle\left\{a_{t}: t \in T_{\eta}, b_{i} \mid \xi \subseteq t\right\}\right\rangle .
$$

For $\eta=\theta$ this follows from the choice of $\theta$. As the sequence $\left(B_{i}^{\eta}\right)_{\eta \leq \beta}$ is continuous and nondecreasing, the inductive step for limit ordinals $\eta$ is trivial. So suppose we are given $\eta=\delta+1$ and in $V^{P_{\delta}}$ we have that $X \mid \xi$ is a maximal antichain in $P\left(B_{1}^{\delta},\left\langle u_{b_{1} \mid \xi}\right\rangle^{F i}, n\right) \times \ldots \times P\left(B_{k}^{\delta},\left\langle u_{b_{k} \mid \xi}\right\rangle^{F i}, n\right)$.

We will use lemma 7.4 for this preservation argument. For this we need to know that $B_{i}^{\eta}$ 's are generated over $B_{i}^{\delta}$ 's like $B_{i}$ 's over $A_{i}$ 's from Lemma 7.4. By the definition of $P^{\eta}$ in $V^{P_{\eta}}$, the algebra $B_{i}^{\eta}$ is generated over the algebra $B_{i}^{\delta}$ by the set

$$
\left\{a_{G(b)}: b_{i} \mid \xi \subseteq b, b \text { is a maximal countable branch of } T_{\delta}, b \in V^{P_{\delta}}\right\} .
$$

Since $b_{i}\left|\xi \neq b_{j}\right| \xi$ for $i<j \leq k$, the product over $i \leq k$ of the products of the forcings $P\left(A_{b}, u_{b}\right)$ for $b$ such that $b_{i} \mid \xi \subseteq b$ and $b$ is a maximal, countable branch 
of $T_{\delta}$ which belongs to $V^{P_{\delta}}$ is a subproduct of the first coordinate of $P^{\delta^{\prime}}$; call this subproduct $P$. Thus by lemma 7.4 in $V^{P_{\delta} * P}$ the $X \mid \xi$ stays maximal antichain in the forcing $P\left(B_{1}^{\delta},\left\langle u_{b_{1} \mid \xi}\right\rangle^{F i}, n\right) \times \ldots \times P\left(B_{k}^{\delta}, u_{b_{k} \mid \xi}, n\right)$. This completes the proof of !).

To finish the proof of the subclaim we need to note that $X \mid \xi$ is a subset of

$$
P\left(A_{b_{1}}, u_{b_{1}}, n\right) \times \ldots \times P\left(A_{b_{k}}, u_{b_{k}}, n\right)
$$

and this forcing is a suborder of $P\left(B_{1}^{\beta},\left\langle u_{b_{1} \mid \xi}\right\rangle^{F i}, n\right) \times \ldots \times P\left(B_{k}^{\beta},\left\langle u_{b_{k} \mid \xi}\right\rangle^{F i}, n\right)$. So $X \mid \xi$ must be a maximal antichain of

$$
P\left(A_{b_{1}}, u_{b_{1}}, n\right) \times \ldots \times P\left(A_{b_{k}}, u_{b_{k}}, n\right),
$$

so $X=X \mid \xi$, but $X \mid \xi$ is countable as $b_{i} \mid \xi$ are countable, so this contradicts the choice of $X$ and this completes the proof of subclaim, so this completes the proof of Claim 1, for the successor of a limit ordinal of cofinality $\omega_{1}$. But since Claim 2 does not say anything in the case of a successor, this finishes the proof of both claims in this case.

So we are left with the proof of the claims for $\alpha$ such that $\alpha=\beta+1$ and $c f(\beta) \neq \omega_{1}$. Work in $V^{P_{\beta}}$. Again for claim 1 it is enough to establish that $P^{\beta}$ is Souslin free in $V^{P_{\beta}}$. It is enough to prove that all forcings of the form $P\left(A_{b}, u_{b}\right)$ for a maximal branch $b$ of $T_{\beta}$ are $\sigma$-centered. Certainly, it is true for countable maximal branches, since then the corresponding forcings are countable as well. Consider, then, an uncountable branch $b$. We will first prove that $b \subseteq T_{\gamma}$ for some $\gamma<\beta$. If $c f(\beta)>\omega_{1}$, this is clear. If $c f(\beta)<\omega_{1}$, if it is not the case, then for every $\gamma<\beta$ the intersection $b \cap T_{\gamma}$ is countable, but then $T_{\beta} \cap b=b$ is countable since the cofinality of $\beta$ was assumed to be countable, so indeed $b \subseteq T_{\gamma}$ for some $\gamma<\beta$. Now, since $b$ is a maximal uncountable branch of $T_{\beta}$, it is a maximal uncountable branch of $T_{\gamma}$ for the $\gamma$ such that $b \subseteq T_{\gamma}$. Now conclude that $b \in V^{P_{\gamma}}$; indeed it follows from the fact that in $V^{P_{\gamma}}$ the forcing $P_{[\gamma, \beta)}$ is Souslin free by results from [De], so it does not add new uncountable branches to $T_{\gamma}$ by [De]. So, by the definition of $P^{\gamma}$, at the stage $\gamma$ we force with a product of countably many copies of $P\left(A_{b}, u_{b}\right)$. Thus this forcing becomes a $\sigma$-centered forcing in $V^{P_{\gamma+1}}$, so it is of this kind in $V^{P_{\beta}}$, as we are in a case of a successor so claim 2 does not say anything and this completes the proof of both claims.

Now we are ready to conclude the theorem. Since all the forcings satisfy the c.c.c., the obtained iteration satisfies the c.c.c., and hence cardinals are preserved and by the appropriate choice of $\dot{Q}^{\alpha}$ 's $\mathrm{MA}_{\kappa}$ holds in $V^{P_{\kappa+}}$. Now, consider in $V^{P{ }^{+}}$ the unions of $T=\bigcup_{\alpha<\kappa^{+}} T_{\alpha}$ and $A=\bigcup_{\alpha<\kappa^{+}} A_{\alpha}$. Then, $A$ is a $T$-algebra and $T$ is a tree of height $\omega_{1}$ with no countable branches (in fact $T=2^{<\omega_{1}}$ ) so there is no ultrafilter of $A$ which is countably generated by fact 2.11. If $b$ is a maximal branch of $T$, then there is $\alpha<\kappa^{+}$such that $b$ is a maximal branch of $T_{\alpha}$ and $b \in V^{P_{\alpha}}$, so by the definition of $P^{\alpha}$ we force at stage $\alpha$ with a product of countably many copies of the forcing $P\left(A_{b}, u_{b}\right)$. Hence this forcing becomes $\sigma$-centered in $V^{P_{\alpha+1}}$ and it is $\sigma$-centered in the final model. The countable tightness of $A$ follows from fact 3.4. This completes the proof of the theorem.

As we mentioned in the introduction, our main interest is to investigate the gaps between certain cardinal functions on compact spaces. From a certain point of view the topic of this section falls into a more general compact topological issue of finding properties of a compact space which imply the existence of points of countable 
character. We found an application of the algebra obtained in this section in this area.

D. Shahmatov in his paper ([Shah] section 4) lists some questions whose equivalent versions are of the form If $X$ is compact and $X$ has a property $\mathcal{P}$, does it imply (consistently / in ZFC) that $X$ has a first countable point? One of them, namely, 4.6., due to Archangel'skii is : Let $X$ be a first countable compact space and $Y$ a continuous image of $X$. Must $Y$ contain a point of countable character?

It is clear that $Y$ as above is countably tight; thus under PFA (by the result from [Do]) it must contain a point of countable character. The following theorem together with the construction described in theorem 7.5, provides a consistently negative answer to the Archangel'skii's question.

Theorem 7.6. Assume $M A_{\kappa}$. Suppose that $A$ is a Boolean algebra such that for every ultrafilter $u \subseteq A$, there is a subalgebra $A_{u} \subseteq A$, such that $u$ is generated from $A_{u},\left|A_{u}\right| \leq \kappa$ and the forcing $P\left(A_{u}, u\right)$ satisfies the c.c.c. Then there is a Boolean algebra $C$ such that $A$ is a subalgebra of $C$ and $\chi(C)=\omega$.

Proof. For every ultrafilter $u \subseteq A$, consider the forcing $P^{\omega}\left(A_{u}, u\right)$, i.e., the countable product with finite supports of forcings $P\left(A_{u}, u\right)$.

Let $b_{1}, b_{2} \in u^{+} \cap A_{u}$ be disjoint. Then the set

$$
D_{b_{1}, b_{2}}=\left\{p \in P^{\omega}\left(A_{u}, u\right): \exists n p_{-1}(n) \supseteq b_{1}, p_{1}(n) \supseteq b_{2}\right\}
$$

is a dense subset of $P\left(A_{u}, u\right)$, where $\left(p_{-1}(n), p_{1}(n)\right)$ is the $n$-th coordinate of $p$. For every ultrafilter $u$ of $A$, find $G_{u} \subseteq P^{\omega}\left(A_{u}, u\right)$ which is a generic set meeting all the sets $D_{b_{1}, b_{2}}$ for disjoint $b_{1}, b_{2}$ in $u^{+} \cap A_{u}$ and all the dense sets from the family $\left\{D_{a}: a \in A_{u}\right\}$ defined in fact 3.2. This is possible since $\mathrm{MA}_{\kappa}$ holds and $\left|A_{u}\right| \leq \kappa$. Let

$$
b(u, n)=\bigcup\left\{a: \exists p \in G_{u} p_{1}(n)=a\right\} .
$$

Now let $C$ be the algebra generated over $A$ by $\{b(u, n): n<\omega, u \in U l t(A)\}$ and let $C_{u}$ be the algebra generated over $A_{u}$ by $\{b(u, n): n<\omega\}$ for any ultrafilter $u$.

Claim. If $v \in U l t(C)$, then $v$ is generated from $C_{v \cap A}$.

Proof. Since $v \cap A$ is generated from $A_{v \cap A}$, it is clear that $v$ is generated from $\left\langle A_{v \cap A} \cup\{b(u, n): n<\omega, u \in \operatorname{Ult}(A)\}\right\rangle$. It is enough to prove for $u \in \operatorname{Ult}(A)-$ $\{v \cap A\}$ that if $b(u, n) \in v$, then there is $b \in A_{v \cap A} \cap v$ such that $b \subseteq b(u, n)$.

Let $a \in(v \cap A)-u$. The element $b(u, n)$ is minimal for $\left(A_{u}, u\right)$; hence it is minimal for $(A, u)$ by lemma 2.4 , so by proposition $2.2, b(u, n) \cap a \in A$. On the other hand $b(u, n) \in v$, so $b(u, n) \cap a \in v$ and finally $b(u, n) \cap a \in A \cap v$, i.e., there is $b \in A_{A \cap v} \cap v$ such that $b \subseteq b(u, n) \cap a \subseteq b(u, n)$ which completes the proof of the claim.

Fix $v \in U l t(C)$. We will prove that it has a countable character. Let $\left\{\varepsilon_{n}: n<\right.$ $\omega\} \in\{-1,1\}^{\omega}$ be such that $b(v \cap A, n)^{\varepsilon_{n}} \in v$. Now consider two cases:

Case 1. For every $a \in A_{v \cap A}-v$ there is $n<\omega$ such that $a \cap b(v \cap A, n)^{\varepsilon_{n}}=\emptyset$.

In this case $\left\{b(v \cap A, n)^{\varepsilon_{n}}: n<\omega\right\}$ is a countable base for $v$. To see this take any $b \in v \cap A$. We may w.l.o.g. assume that $b \in A_{v \cap A}$, so there is $n<\omega$ such that $b^{-1} \cap b(v \cap A, n)^{\varepsilon_{n}}=\emptyset$, so $b(v \cap A, n)^{\varepsilon_{n}} \subseteq b$. So by the claim, $v$ has countable character. 
Case 2. There is $a \in A_{v \cap A}-v$ such that for every $n<\omega$ we have $a \cap b(v \cap A, n)^{\varepsilon_{n}} \neq \emptyset$.

In this case $\left\{b(v \cap A, n)^{\varepsilon_{n}} \cap a^{-1}: n<\omega\right\}$ is a countable base for $v$. To see this take any $b \in v \cap A$, we may w.l.o.g. assume that $b \in A_{v \cap A}$. Now $G_{v \cap A}$ meets $D_{b^{-1} \cap a^{-1}, a}$, i.e., there are $n<\omega$ and $\delta \in\{-1,1\}$ such that $b^{-1} \cap a^{-1} \subseteq$ $b(v \cap A, n)^{-\delta \varepsilon_{n}}$, and $a \subseteq b(v \cap A, n)^{\delta \varepsilon_{n}}$. By the assumed property of $a$, we obtain that $\delta=1$, so $b(v \cap A, n)^{\varepsilon_{n}} \subseteq\left(b^{-1} \cap a^{-1}\right)^{-1}=b \cup a, b(v \cap A, n)^{\varepsilon_{n}} \cap a^{-1} \subseteq b$, so by the claim, $v$ has countable character.

\section{Building SUPERATOMIC ALGEBRAS ALONG DIRECTED SETS}

The purpose of this section is to support the following section where we obtain some consistency results. Recall that a Boolean algebra is called minimally generated if and only if it is generated by $\left(a_{\alpha}: \alpha<\beta\right)$ and each $a_{\alpha}$ is minimal for $\left(\left\langle\left\{a_{\alpha^{\prime}}: \alpha^{\prime}<\alpha\right\}\right\rangle, u_{\alpha}\right)$ where $u_{\alpha}$ is some ultrafilter of $\left\langle\left\{a_{\alpha^{\prime}}: \alpha^{\prime}<\alpha\right\}\right\rangle$. If $u_{\alpha^{\prime}} \subseteq u_{\alpha}$ for $\alpha^{\prime} \leq \alpha$, then the obtained algebra is superatomic (see [Kop1]). W.l.o.g. we may assume that $u_{\alpha}=\left\langle\left\{a_{\alpha^{\prime}}: \alpha^{\prime}<\alpha\right\}\right\rangle^{F i}$. Also note that minimality then means that

$$
a_{\alpha} \cap a_{\alpha^{\prime}} \in\left\langle\left\{a_{\alpha^{\prime \prime}}: \alpha^{\prime \prime}<\alpha^{\prime}\right\}\right\rangle .
$$

One can impose more restricting conditions on the subalgebra in which $a_{\alpha} \cap a_{\alpha^{\prime}}$ should be, obtaining more structural information about the obtained algebra. In [BS], Baumgartner and Shelah considered a function $f:[\beta]^{2} \rightarrow[\beta]^{\omega}$ as a tool for imposing such restrictions. In this section we consider a partial order on $\beta$ for this purpose. Later, using a particular partial order, we will construct a forcing notion which adds a Boolean algebra of some interest. In this section working with ideals will be more convenient than working with filters.

Definition 8.1. Let $P$ be a well-founded partial order. We say that a Boolean algebra $A$ is a $P$-superatomic algebra if and only if $A=\left\langle\left\{a_{X}: X \in P\right\}\right\rangle, I_{A}=$ $\left\langle\left\{a_{X}: X \in P\right\}\right\rangle_{A}^{I d}$ forms a proper ideal of $A$ and for every $X, Y \in P$ we have

1) $a_{X} \notin\left\langle\left\{a_{Z}: Z<X\right\}\right\rangle$.

2) $a_{X} \cap a_{Y} \in\left\langle\left\{a_{Z}: Z \leq X, Y\right\}\right\rangle$.

If $F \subseteq P$, then by $A_{F}$ we denote $\left\langle\left\{a_{X}: X \in F\right\}\right\rangle_{A}$ and by $I_{F}$ we denote $\left\langle\left\{a_{X}: X \in\right.\right.$ $F\}\rangle_{A_{F}}^{I \bar{d}}$. By an $F$-superatomic algebra we mean a $(P \mid F)$-superatomic algebra, if $P$ is clear from the context. If we consider many $P$-superatomic algebras, then we denote the element $a_{X}$ of $A$ by $a_{X}(A)$ and $I_{F}$ by $I_{F}(A)$, in order to avoid confusion.

If $R$ is a suborder of $P$ and $B$ is an $R$-superatomic algebra and $A$ is a $P$ superatomic algebra, then we say that $B \leq A$ if and only if there is an embedding of Boolean algebras $i: B \rightarrow A$ such that $i\left(a_{X}(B)\right)=a_{X}(A)$ for all $X \in R$. If $i$ is onto, then we write $A \equiv B$. If $B \leq A$, we will often identify $a_{X}(B)$ with $a_{X}(A)$ for $X \in R$

Fact 8.2. Let $P$ be a partial order and $G$ a downward closed subset of $P$ and let $A$ be $P$-superatomic algebra. Then $A_{G}=\left\{a_{X}(A): X \in G\right\}$ is an $G$-superatomic algebra such that $A_{G} \leq A$.

Proof. The existence of the embedding as in definition 8.1 is obvious. So, we are left with the proof that $A_{G}$ is a $G$-superatomic algebra. The only nontrivial part is to prove that 2) of definition 8.1 holds. Take $X, Y \in G$; then use that fact that $G$ is downward closed i.e., $\left\{a_{Z}\left(A_{G}\right): Z \leq_{P \mid G} X, Y\right\}=\left\{a_{Z}(A): Z \leq_{P} X, Y\right\}$.

If $P$ is a partial order and $X \in P$, then $P \mid X$ we will denote the order restricted to the set $\left\{Y<_{P} X: Y \in P\right\}$. 
Fact 8.3. Suppose that $A$ is a $P$-superatomic algebra; then for every $X \in P$ we have $I_{P \mid X \cup\{X\}}=\left\langle I_{P \mid X \cup\{X\}}\right\rangle_{A}^{I d}$.

Proof. Let $a \in\left\langle I_{P \mid X \cup\{X\}}\right\rangle_{A}^{I d}$; then there is $a_{0}=\bigcup_{i<k} a_{X_{i}}$ such that $a \subseteq a_{0}$ and $X_{i} \leq X$ for $i<k \in \omega$. But then, $a=\bigcup_{i<k}\left(a \cap a_{X_{i}}\right)$. So it is enough to show that $a_{Y} \cap a \in A_{P \mid X \cup\{X\}}$ for $Y \leq X$. Let

$$
a=\bigcup_{i<k} \bigcap_{Z \in G} a_{Z}^{\varepsilon(Z, i)}
$$

for some $G \in[P]^{<\omega}, \varepsilon(Z, i) \in\{-1,0,1\}$. Again, it is enough to show that $a_{Z}^{\varepsilon(Z, i)} \cap$ $a_{Y} \in A_{P \mid X \cup\{X\}}$ for any $Y \in P \mid X \cup\{X\}$. If $\varepsilon(Z, i)=1$, we use part 2) of Definition 8.1. If $\varepsilon(Z, i)=0$, it is trivial. If $\varepsilon(Z, i)=-1$. Then $a_{Z}^{-1} \cap a_{Y}=a_{Y}-\left(a_{Y} \cap a_{Z}\right)$ but $a_{Y} \cap a_{Z} \in A_{P \mid X \cup\{X\}}$ by Definition 8.1. Hence we are done.

Fact 8.4. Let $A$ be a $P$-superatomic algebra and $F \subseteq P$ be a downward closed subset of $P$. Then $I_{F}=\left\langle I_{F}\right\rangle_{A}^{I d}$.

Proof. Let $a \in\left\langle I_{F}\right\rangle_{A}^{I d}$, then there is $a_{0}=\bigcup_{i<k} a_{X_{i}}$ such that $a \subseteq a_{0}$ and $X_{i} \in F$ for $i<k \in \omega$. But then $a=\bigcup_{i<k}\left(a \cap a_{X_{i}}\right)$ and by Fact 8.3, $a_{X_{i}} \cap a_{0} \in I_{P \mid X_{i} \cup\left\{X_{i}\right\}} \subseteq I_{F}$, so $a \in I_{F}$.

Fact 8.5. Suppose that $P$ is a partial order, and $F, G \subseteq P$ are downward closed sets. Then $I_{F}(A) \cap I_{G}(A)=I_{F \cap G}(A)$ for a $P$-superatomic algebra $A$.

Proof. Obviously $I_{F \cap G} \subseteq I_{F} \cap I_{G}$. So, take $a \in I_{F} \cap I_{G}$. Suppose that

$$
a=\bigcup_{i<k} \bigcap_{X \in F^{\prime}} a_{X}^{\varepsilon(X, i)}, a=\bigcup_{j<m} \bigcap_{Y \in G^{\prime}} a_{Y}^{\delta(Y, j)}
$$

for some $F^{\prime} \in[F]^{<\omega}, G^{\prime} \in[G]^{<\omega}$ and $\varepsilon(X, i), \delta(Y, j) \in\{-1,0,1\}, k, m<\omega$. Note that since $a \in I_{F}, I_{G}$, for every $i<k, j<m$ there are $X_{i} \in F^{\prime}, Y_{j} \in G^{\prime}$ such that $\varepsilon\left(X_{i}, i\right), \delta\left(Y_{j}, j\right)=1$. So,

$$
a=a \cap a=\bigcup_{(i, j) \in k \times m}\left[\bigcap_{(X, Y) \in F^{\prime} \times G^{\prime}}\left(a_{X}^{\varepsilon(X, i)} \cap a_{Y}^{\delta(Y, j)}\right) \cap a_{X_{i}} \cap a_{Y_{j}}\right] .
$$

But (by Definition 8.1) $a_{X_{i}} \cap a_{Y_{j}} \in\left\langle\left\{a_{X}: X \leq X_{i}, Y_{j}\right\}\right\rangle$, so $a_{X_{i}} \cap a_{Y_{j}} \in I_{\left\{X: X \leq X_{i}, Y_{j}\right\}}$ $\subseteq I_{F \cap G}$ since $F, G$ are downward closed. Now use Fact 8.4, to conclude that $a \in I_{F \cap G}$.

Fact 8.6. Suppose that $P$ is a well-founded partial order and that $A$ is a $P$-superatomic algebra. Then $A$ is a minimally generated Boolean algebra which is superatomic.

Proof. Let $\left\{X_{\xi}: \xi<\theta\right\}$ be a well-ordering of $P$ such that if $\xi_{1} \leq \xi_{2}$, then $\operatorname{rank}\left(X_{\xi_{1}}\right) \leq \operatorname{rank}\left(X_{\xi_{2}}\right)$. We prove that $a_{X_{\xi}}$ is minimal for $\left(A_{\xi}, I_{\xi}^{+}\right)$, where $A_{\xi}=$ $\left\langle\left\{a_{X_{\eta}}: \eta<\xi\right\}\right\rangle$ and $I_{\xi}=\left\langle\left\{a_{X_{\eta}}: \eta<\xi\right\}\right\rangle_{A_{\xi}}^{I d}$.

Obviously, $\left\langle\left\{a_{X_{\eta}}: \eta<\xi\right\}\right\rangle_{A_{\xi}}^{I d}=I_{\xi}$ is a maximal ideal of $A_{\xi}$. So it is enough to show that $I_{\xi} \subseteq\left\{a \in A_{\xi}: a \cap a_{X_{\xi}} \in A_{\xi}\right\}$. Let $a \in I_{\xi}$. Then for some $F \in$ $\left[\left\{X_{\eta}: \eta<\xi\right\}\right]^{<\omega}, k \in \omega$, we have

$$
a=\bigcup_{j<k} \bigcap_{X \in F} a_{X}^{\varepsilon(X, j)}
$$


for some $\varepsilon(X, j) \in\{-1,0,1\}$ such that for every $j<k$ there is $X_{j} \in F$ that $\varepsilon\left(X_{j}, j\right)=1$. So

$$
a \cap a_{X_{\xi}}=\bigcup_{j<k}\left(\bigcap_{X \in F} a_{X}^{\varepsilon(X, j)} \cap\left(a_{X_{j}} \cap a_{X_{\xi}}\right)\right)
$$

but $a_{X_{j}} \cap a_{X_{\xi}} \in\left\langle I_{P \mid X_{\xi}}\right\rangle_{I d}^{A}=I_{P \mid X_{\xi}} \subseteq I_{\left\{X_{\eta}: \eta<\xi\right\}}=I_{\xi}$, by fact 8.4. Hence $a \cap a_{X_{\xi}} \in$ $I_{\xi} \subseteq A_{\xi}$; thus $a_{X_{\xi}}$ is minimal for $\left(A_{\xi}, I_{\xi}^{+}\right)$. Superatomicity of $A$ follows from Proposition 3.5 from [Kop1].

Fact 8.7. Let $P$ be a well-founded partial order and let $A$ be a $P$-superatomic algebra. Let $u$ be an ultrafilter of $A$. Then, either

1) $u=\infty_{A}=\left\langle\left\{a_{X}^{-1}: X \in P\right\}\right\rangle_{A}^{F i}$ or

2) $u=u_{Y}=\left\langle\left\{a_{Y}\right\} \cup\left\{a_{X}^{-1}: X<Y\right\}\right\rangle_{A}^{F i}$ for some $Y \in P$.

Every filter of the above form $u_{Y}$ is an ultrafilter of $A$.

Proof. If $u \subseteq \infty_{A}$, then we are done since $\infty_{A}$ is a proper filter by definition 8.1 and it is an ultrafilter of $A$ because it is generated by generators of $A$.

Suppose that $u \neq \infty_{A}$, i.e., for some $Y \in P$ we have $a_{Y} \in u$. Let $Y$ be an element of $P$ of minimal rank among elements $Y$ such that $a_{Y} \in u$. By the minimality $u_{Y} \subseteq u$. So, we are left with the proof that $u_{Y}$ is an ultrafilter for each $Y \in P$. First note that $u_{Y}$ is a proper filter; if not then $a_{Y} \subseteq a_{X_{1}} \cup \cdots \cup a_{X_{n}}$ for some $X_{1}, \ldots, X_{n}<Y$, so $a_{Y} \in\left\langle I_{Y}\right\rangle_{A}^{I d}=I_{P \mid Y} \subseteq A_{P \mid Y}$, by fact 8.4, but this contradicts definition 8.1.

Now note that if $a \cap a_{Y} \in I_{P \mid Y}$ for $a \in A$, then there are $X_{1}, \ldots, X_{n}<Y$ such that $a \cap a_{Y} \subseteq a_{X_{1}} \cup \cdots \cup a_{X_{n}}$, i.e., $a_{X_{1}}^{-1} \cap \ldots \cap a_{X_{n}}^{-1} \supseteq a^{-1} \cup a_{Y}^{-1}$ so $a^{-1} \in u_{Y}$.

So, it is enough to note that either $a \cap a_{Y} \in I_{P \mid Y}$ or $a^{-1} \cap a_{Y} \in I_{P \mid Y}$ for any $a \in A$.

For this note that $a \cap a_{Y}, a^{-1} \cap a \in I_{P \mid Y \cup\{Y\}} \subseteq A_{P \mid Y \cup\{Y\}}$, so there are $b_{1}, b_{-1} \in$ $A_{P \mid Y}$ such that $a^{\varepsilon} \cap a_{Y}=b_{\varepsilon} \cap a_{Y}$ for $\varepsilon \in\{-1,1\}$.

If $b_{-1}, b_{1} \notin I_{P \mid Y}$, then, as $I_{P \mid Y}$ is a maximal ideal in $A_{P \mid Y}$, we have $b_{-1} \cap b_{1} \notin$ $I_{P \mid Y}$ so $I_{P \mid Y} \ni b_{-1}^{-1} \cup b_{1}^{-1} \supseteq a_{Y}$, because $a_{Y} \cap b_{-1} \cap b_{1}=\emptyset$. Hence by fact 8.4, $a_{Y} \in I_{P \mid Y} \supseteq A_{P \mid Y}$ and this contradicts definition 8.1. The obtained contradiction proves that for some $\varepsilon \in\{-1,1\}$, we have $b_{\varepsilon} \in I_{P \mid Y}$, but then $a^{\varepsilon} \cap a \in I_{P \mid Y}$, as required.

Fact 8.8. Suppose $P$ is a partial order whose cofinality is $\kappa$. Then $\chi\left(\infty_{A}, A\right) \geq \kappa$.

Proof. Suppose that $\chi\left(\infty_{A}, A\right)<\kappa$, i.e., there is some $Q \in[P]^{<\kappa}$ such that $\left\langle\left\{a_{X}^{-1}\right.\right.$ : $X \in Q\}\rangle_{A}^{F i}$ generate $\infty_{A}$. Since $Q$ is not cofinal in $P$, there is $Y \in P$ such that for no $X \geq Y$ we have $X \in Q$. Find $F \in[Q]^{<\omega}$ such that $a_{Y}^{-1} \supseteq \bigcap_{X \in F} a_{X}^{-1}$; hence

$$
a_{Y}=a_{Y} \cap \bigcup_{X \in F} a_{X} \in\left\langle\left\{a_{Z}: Z<Y\right\}\right\rangle
$$

by the choice of $Y$. But this contradicts 1) of Definition 8.1.

\section{Hereditary Characters of points in COMPaCt SPACES}

In this section we consider forcing a $P$-superatomic algebra with finite conditions which are $F$-superatomic algebras for $F \in[P]^{<\omega}$. 
Definition 9.1. Let $P$ be a well-founded partial order. We define $\mathcal{P}_{P}$ to be a notion of forcing consisting of conditions of the form $\left(\operatorname{supp}(p), A_{p}\right)$ where $\operatorname{supp}(p) \in[P]<\omega$ and $A_{p}=\left\langle\left\{a_{X}: X \in \operatorname{supp}(p)\right\}\right\rangle$ is a $\operatorname{supp}(p)$-superatomic algebra. We define the order on $\mathcal{P}_{P}$ by putting $q \leq p$ if and only if

1) $\operatorname{supp}(q) \supseteq \operatorname{supp}(p)$,

2) $A_{p} \leq A_{q}$.

Now let us prove some lemmas concerning creating some conditions in $\mathcal{P}_{P}$. For next three lemmas we fix some $P$ and $\mathcal{P}$ as in definition 9.1. In the proofs of these lemmas we often identify our abstract algebras that are parts of conditions of $P$ with some representations of these algebras as fields of sets. Often we change these interpretations if it is convenient for the proof.

Lemma 9.2. For all $p \in \mathcal{P}_{P}$, for all $X \in P$ there is $q \leq p$ such that $X \in \operatorname{supp}(q)$.

Proof. Let $A_{p} \subseteq \wp(W)$ for some set $W$. We may w.l.o.g. assume that $\bigcap I_{\operatorname{supp}\left(A_{p}\right)}^{+}=$ $V \subseteq W$ is of cardinality bigger than one. Let $v \in V$, put $a_{X}=\{v\}$, and extend $a_{p}$ to $a_{q}$ by adding $a_{X}$.

Lemma 9.3. Suppose that $p \in P$, $\sup p(p)=F$. Let $G \subseteq F$ be downward closed in $F$; then $p \mid G=\left(G, A_{G}\right)$ is a condition of $P$ such that $p \leq p \mid G$.

Proof. Note that by fact $8.2, A_{G} \leq A$.

Lemma 9.4. Suppose that $F \cap G \in[P]^{<\aleph_{0}}$ are such that $F \cap G$ is downward closed in $G$. Suppose that $A, B$ are $F$-superatomic and $G$-superatomic algebras respectively, such that there is an $(F \cap G)$-superatomic algebra $C$ such that $C \leq A, B$. Then there is an $(F \cup G)$-superatomic algebra $D$ such that $A, B \leq D$ and for every $X \in G-F$

$$
a_{X}(D) \cap \bigcup I_{F}(D)=a_{X}(D) \cap \bigcup I_{F \cap G}(D) .
$$

Lemma 9.5. Let $C \leq A$ be $F$-superatomic and $G$-superatomic algebras respectively. Let $F \subseteq G \subseteq \mathcal{F} \mid Z$, for some $Z \in P$. Suppose that $B$ is an $(F \cup\{Z\})$ superatomic algebra such that $C \leq B$. Then there is an $(F \cup\{Z\})$-superatomic algebra $D$ such that $A, B \leq D$ and $\bigcup I_{G}-\bigcup I_{F} \subseteq a_{Z}$.

Proof. Suppose that $A_{0}$ is an $F_{0}$-superatomic algebra and $F_{0}$ has a maximal element $Z_{0}$. Then $a_{Z_{0}}^{-1} \cap a_{X}=a_{X}-\left(a_{Z_{0}} \cap a_{X}\right) \in\left\langle\left\{a_{Y}: Y \leq Z_{0}, X\right\}\right\rangle$ because $X \leq Z_{0}$. And so we have another minimally generated algebra (isomorphic to the original but with different minimal generators) $A_{0}^{*}$ which is minimally generated by $\left\langle\left\{a_{X}: X \in F-\left\{Z_{0}\right\}\right\} \cup\left\{a_{Z_{0}}^{-1}\right\}\right\rangle$, (i.e., $\left.a_{Z_{0}}\left(A_{0}^{*}\right)=a_{Z_{0}}^{-1}\left(A_{0}\right)\right)$. So, consider $B^{*}$ for $B$ from the assumptions of the lemma. Since $F \subseteq G$ we may apply lemma 9.4 , to get an algebra $D_{0}$ such that $A, B^{*} \leq D_{0}$ and $\bigcup I_{G} \cap a_{Z}\left(D_{0}\right)=\bigcup I_{F} \cap a_{Z}\left(D_{0}\right)$. Now consider $D_{0}^{*}$; obviously $\bigcup I_{G}-\bigcup I_{F} \subseteq a_{Z}\left(D_{0}^{*}\right)$ and $A \leq D_{0}^{*}$. Also $B \leq D_{0}^{*}$ since $B=\left\langle C \cup\left\{a_{Z}\right\}\right\rangle$.

Fact 9.6. Suppose that $P$ is locally countable, i.e., $P \mid X=\left\{Y: Y{ }_{P} X\right\}$ is countable for every $X \in P$. Then $\mathcal{P}_{P}$ is a c.c.c. notion of forcing.

Proof. Suppose not, i.e., that there is an antichain $\left(p_{\xi}: \xi<\omega_{1}\right)$. Use the standard $\Delta$-system argument and local countability of $P$ to conclude that we may assume that $\left(\operatorname{supp}\left(p_{\xi}\right): \xi<\omega_{1}\right)$ forms a $\Delta$ system with the root $\Delta$ which is downward closed in the union of the $\Delta$-system and $A_{p_{\xi_{1}}}\left|\Delta \equiv A_{p_{\xi_{2}}}\right| \Delta$ for any $\xi_{1}, \xi_{2}<\omega_{1}$. Now apply Lemma 9.4 to amalgamate any two conditions. 
Fact 9.7. Suppose $P$ is a well-founded and locally countable partial order. Then $\mathcal{P}_{P} \Vdash A_{P}$, the direct limit of $\left(A_{p}: p \in \dot{G}\right)$, is a $\check{P}$-superatomic algebra, where $\dot{G}$ is the canonical name for a generic set in $\mathcal{P}_{P}$.

Fact 9.8. Suppose that $P$ is a well-founded, locally countable partial order. Then $\mathcal{P}_{P} \Vdash P\left(A_{P}, \infty_{A_{P}}\right)$ is a c.c.c. forcing notion.

Proof. Suppose the opposite, i.e., let $p \|-\left\{\left(p_{-1}^{\xi}, p_{1}^{\xi}\right): \xi<\omega_{1}\right\}$ be an antichain of $P\left(A_{P}, \infty_{A_{P}}\right)$. Choose $\left(p_{\xi}: \xi<\omega_{1}\right) \subseteq P$ such that there are Boolean polynomials $\phi_{i}$ for $i \in\{-1,1\}$ and there are $n \in \omega$ and $\left(R_{\xi} \in[P]^{n}: \xi<\omega_{1}\right)$ such that

$$
p_{\xi} \Vdash\left(p_{-1}^{\xi}, p_{1}^{\xi}\right)=\left(\phi_{-1}^{\xi}\left(a_{R_{\xi}}\right), \phi_{1}^{\xi}\left(a_{R_{\xi}}\right)\right)
$$

where $\phi\left(a_{F}\right)$ denotes a polynomial $\phi$ in variables $\left\{a_{X}: X \in F\right\}$. Note that we also may w.l.o.g. assume that $\phi_{1}^{\xi}=\phi_{1}, \phi_{-1}^{\xi}=\phi_{-1}$ for some polynomials $\phi_{1}$ and $\phi_{-1}$. We may w.l.o.g. assume that $R_{\xi}$ 's form a $\Delta$-system with a root $\Delta$, which is downward closed in every $R_{\xi}$ (by local countability of $P$ ). We may w.l.o.g. assume that for every $\xi \in \omega_{1}, R_{\xi} \subseteq \operatorname{supp}\left(p_{\xi}\right)$ and that there is a single $\Delta$-superatomic algebra $C$ on $\Delta$, such that $C \leq A_{p_{\xi}}$ for every $\xi<\omega_{1}$. Moreover, we can assume that for every $\xi_{1}, \xi_{2}<\omega_{1}$ there is an order isomorphism $\pi: R_{\xi_{1}} \rightarrow R_{\xi_{2}}$ such that $\pi \mid \Delta=i d_{\Delta}$, which extends to an isomorphism of superatomic algebras $A_{p_{\xi_{1}}}, A_{p_{\xi_{2}}}$, i.e., there is an isomorphism $j: A_{p_{\xi_{1}}} \rightarrow A_{p_{\xi_{2}}}$ such that $j\left(a_{X}\left(A_{p_{\xi_{1}}}\right)\right)=a_{\pi(X)}\left(A_{p_{\xi_{2}}}\right)$. Choose $\xi_{1} \neq \xi_{2}$ and apply lemma 9.4 , to find $D$ such that $A_{p_{\xi_{1}}}, A_{p_{\xi_{2}}} \leq D$ such that for every $X \in R_{\xi_{1}}-\Delta$ we have $a_{X}\left(A_{p_{\xi_{1}}}\right) \cap I_{R_{\xi_{2}}}=a_{X}\left(A_{p_{\xi_{1}}}\right) \cap \bigcup I_{\Delta}$. We will show that $q \Vdash\left(p_{-1}^{\xi_{1}} \cup p_{-1}^{\xi_{2}}, p_{1}^{\xi_{1}} \cup p_{1}^{\xi_{2}}\right) \in P\left(A_{P}, \infty A_{P}\right)$, where $A_{q}=D$.

Note that $p_{\xi_{1}} \Vdash \phi_{i}\left(a_{R_{\xi_{1}}}\right) \subseteq \bigcup I_{R_{\xi_{1}}}$, since $p_{\xi_{1}} \Vdash \phi_{i}\left(a_{R_{\xi_{1}}}\right) \notin \infty$. Hence $\phi_{i}\left(a_{R_{\xi_{1}}}\right) \cap$ $\bigcup I_{R_{\xi_{2}}}=\phi_{i}\left(a_{R_{\xi_{1}}}\right) \cap \bigcup I_{\Delta}$. By the isomorphism $j$ and the fact that $\left\langle I_{\Delta}\right\rangle_{D}^{I d}=I_{\Delta}$, we have $\phi_{i}\left(a_{R_{\xi_{1}}}\right) \cap \bigcup I_{\Delta}=\phi_{i}\left(a_{R_{\xi_{2}}}\right) \cap \bigcup I_{\Delta}$ for $i=-1,1$, so

$$
\begin{gathered}
\phi_{i}\left(a_{R_{\xi_{1}}}\right) \cap \phi_{-i}\left(a_{R_{\xi_{2}}}\right)=\phi_{i}\left(a_{R_{\xi_{1}}}\right) \cap\left(\bigcup I_{\Delta}\right) \cap \phi_{-i}\left(a_{R_{\xi_{2}}}\right) \\
=\phi_{i}\left(a_{R_{\xi_{2}}}\right) \cap\left(\bigcup I_{\Delta}\right) \cap \phi_{-i}\left(a_{R_{\xi_{2}}}\right) \subseteq \phi_{i}\left(a_{R_{\xi_{2}}}\right) \cap \phi_{-i}\left(a_{R_{\xi_{2}}}\right)=\emptyset .
\end{gathered}
$$

This completes the proof of the lemma.

Before entering the main construction of this section let us prove an elementary fact concerning hereditary characters of points in compact spaces.

Fact 9.9. Let $X$ be a compact space, $x \in X$. Suppose that $h \chi(x, X)=\kappa$; then $c f(\kappa)>\omega$ or $\kappa=\omega$.

Proof. We will show that if $\operatorname{cf}(\kappa)=\omega$, then there is a converging sequence to $x$; hence $h \chi(x, X)=\omega$. We may w.l.o.g. assume that $\chi(x, X)=\kappa$. Let $\mathcal{B}$ be a neighborhood base at $x$, of open subsets of $X$, such that $\mathcal{B}=\bigcup_{n \in \omega} \mathcal{B}_{n}, \mathcal{B}_{n} \subseteq \mathcal{B}_{n+1}$ and $\left|\mathcal{B}_{n}\right|<\kappa$ for every $n \in \omega$. Since $X$ is compact, the pseudocharacter of $x$ is $\kappa$. So there is $x_{n} \neq x$ such that $x_{n} \in \bigcap \mathcal{B}_{n}$ for every $n \in \omega$. Now note that $\left(x_{n}\right)_{n \in \omega} \rightarrow x$.

In the remaining part of this section we will consider $\mathcal{P}_{P}$ for a particular $P$. To describe properties of $P$ we need to recall some terminology. $\mathcal{F} \subseteq[\lambda]^{\aleph_{0}}$ is called a stationary coding set $[\mathrm{Z}]$ if and only if it is stationary in $[\lambda]^{\aleph_{0}}$ and there is a 1-1 function $c: \mathcal{F} \rightarrow \lambda$ such that for every $X, Y \in \mathcal{F}$ we have

$$
X \subset Y \Rightarrow c(X) \in Y \text {. }
$$


For $\mathcal{F} \subseteq[\lambda]^{\aleph_{0}}$ and $f:[\mathcal{F}]^{<\aleph_{0}} \rightarrow[\lambda]^{\aleph_{0}}$, we say that $X \in \mathcal{F}$ is closed under $f$ if and only if we have $f(Y) \subseteq X$ for every $Y \in[\mathcal{F} \mid X]^{<\aleph_{0}}$. We will consider $\mathcal{P}_{P}$ for $P$ which is a stationary coding set $\mathcal{F}$ together with inclusion. The consistency of the existence of locally countable, well-founded, stationary coding sets for any cardinal $\lambda$ of uncountable cofinality was proved e.g. in [Kosz3]; for more on the existence of stationary coding sets see [Sh3], [Ve]. At the end of this discussion let us note the following elementary

Fact 9.10. Suppose $\lambda$ is an uncountable cardinal and $\mathcal{F} \subseteq[\lambda]^{\aleph_{0}}$ is a stationary coding set. Suppose that $f:[\mathcal{F}]^{<\aleph_{0}} \rightarrow[\lambda]^{\aleph_{0}}$. Then there is $X \in \mathcal{F}$ such that $X$ is closed under $f$.

Proof. Let $c: \mathcal{F} \rightarrow \lambda$ be the coding function. Let $M \prec H(\theta)$ be such that $c, f \in M$ and $M \cap \lambda \in \mathcal{F}$ and $\theta$ is large enough. Take any $Y \in[\mathcal{F} \mid(M \cap \lambda)]^{<\aleph_{0}}$; then $c(Y) \in[M \cap \lambda]^{<\aleph_{0}}$, so $c(Y) \in M$. Since $c$ is $1-1$, we have $Y \in M$, so $f(Y) \in M$, and so $f(Y) \subseteq M \cap \lambda$, as required.

From now on, we fix a cardinal $\lambda$ of uncountable cofinality and $\mathcal{F}$, a well-founded, locally countable stationary coding set in $[\lambda]^{\aleph_{0}}$. By $P$ we mean $\mathcal{P}_{\mathcal{F}}$.

Fact 9.11. $P \| h \chi(\infty)=\lambda$.

Proof. Suppose the opposite, i.e., that there is a $p_{0} \in P$ such that for some $\kappa<\lambda$ we have that $p_{0} \Vdash h \chi(\infty)=\kappa$. It follows that $p_{0}$ forces that there are an ideal $\dot{J} \subseteq \infty^{+}$ and a sequence $\left\{a_{\dot{X}_{\xi}}: \xi<\kappa\right\}$ such that $a_{\dot{X}_{\xi}} \notin \dot{J}_{\xi}$ where $\dot{J}_{\xi}=\left\langle\left\{a_{\dot{X}_{\xi^{\prime}}}: \xi^{\prime}<\xi\right\} \cup \dot{J}\right\rangle$ for $\xi \leq \kappa$ and $\infty^{+}=\dot{J}_{\kappa}$. Since $P$ is c.c.c., there is $\alpha \in \lambda$ such that $p_{0} \Vdash \alpha \notin \bigcup_{\xi<\kappa} \dot{X}_{\xi}$.

By fact 9.9 we may assume that $c f(\kappa)>\omega$ or $\kappa=\omega$. We will consider these two cases separately.

First, assume that $c f(\kappa)>\omega$.

Let $\eta$ be a function from $[\mathcal{F}]^{<\omega}$ into $\kappa$ such that

$$
\eta\left(X_{1}, \ldots, X_{k}\right)=\min \left\{\eta<\kappa: P \Vdash a_{X_{1}} \cup \ldots \cup a_{X_{k}} \in \dot{J}_{\eta}\right\} .
$$

Since $P$ is a c.c.c. notion of forcing and $\left(\dot{J}_{\eta}\right)_{\eta<\kappa}$ is a name for a strictly increasing sequence of ideals of length of uncountable cofinality whose union is the ideal $\infty^{+}$, the existence of such an $\eta$ is easy to prove. Now we are going to use that fact that $\mathcal{F}$ is a stationary coding set. Define a function $f:[\mathcal{F}]^{<\aleph_{0}} \rightarrow \mathcal{F}$ such that for every $Y \in[\mathcal{F}]^{<\omega}$ we have $\alpha \in f(Y)$ and

$$
\left\{X: \exists p \in P p \| X=\dot{X}_{\eta(Y)}\right\} \cup \bigcup\left\{\operatorname{supp}(p): p \in \mathcal{A}_{\eta(Y)}\right\} \subseteq f(Y)
$$

where $\mathcal{A}_{\eta(Y)}$ is a maximal antichain in $P$ deciding the value of $\dot{X}_{\eta(Y)}$. Again, since $P$ is a c.c.c. notion of forcing $f$ can be easily constructed. Now find $Z \in \mathcal{F}$ such that $Z$ is closed under $f$.

Take any $q \leq p_{0}$ and consider the conditions $q_{1}=q\left|(\mathcal{F} \mid Z), q_{2}=q\right|(\mathcal{F} \mid Z \cup\{Z\})$. By lemma 9.2, $q \leq q_{2} \leq q_{1}$. Since $\operatorname{supp}\left(q_{1}\right) \subseteq \mathcal{F} \mid Z$, we have that there is $r \in$ $\mathcal{A}_{\eta\left(\operatorname{supp}\left(q_{1}\right)\right)}$ such that $r \| q_{1}$, supp $(r) \in[\mathcal{F} \mid Z]^{<\omega}$ and $r \| \dot{X}_{\eta\left(\operatorname{supp}\left(q_{1}\right)\right)}=\check{X}$ for some $X \in \mathcal{F} \mid Z$. Take $s \leq r, q_{1}$. By lemma 9.4 we may w.l.o.g. assume that $\operatorname{supp}(s) \in$ $[\mathcal{F} \mid Z]^{<\omega}$ and by lemma 9.3 we may w.l.o.g. assume that $X \in \operatorname{supp}(s)$. Now note that we can apply lemma 9.4 for $C=A_{q_{1}}, A=A_{s}, B=A_{q_{2}}$, to obtain $D$ such that $A_{q_{2}}, A_{s} \leq D$. By lemma $9.4, D$ can be assumed to be an $F$-superatomic algebra 
for $F \in[\mathcal{F} \mid Z \cup\{Z\}]^{<\omega}$. Let $s_{1}$ be such that $A_{s_{1}}=D$. Thus $\operatorname{supp}\left(s_{1}\right) \subseteq \mathcal{F} \mid Z \cup\{Z\}$ and $s_{1} \leq s, q_{2}$ and

$$
s_{1} \Vdash a_{X_{\eta\left(s u p p\left(q_{1}\right)\right)}}-\bigcup I_{s u p p\left(q_{1}\right)} \subseteq a_{Z}
$$

Note that

$$
p \Vdash \bigcup I_{s u p p\left(q_{1}\right)} \in J_{\eta\left(\operatorname{supp}\left(q_{1}\right)\right.}, a_{X_{\eta\left(\operatorname{supp}\left(q_{1}\right)\right)}} \notin J_{\eta\left(\operatorname{supp}\left(q_{1}\right)\right)},
$$

SO

$$
s_{1} \Vdash a_{Z} \notin J_{\eta\left(\operatorname{supp}\left(q_{1}\right)\right)} .
$$

Now use lemma 9.4, to find $s_{2} \leq s_{1}, q$. Since $\operatorname{supp}\left(s_{1}\right) \subseteq \mathcal{F} \mid Z \cup\{Z\}$, supp $(q) \cap$ $\operatorname{supp}\left(s_{1}\right)=\operatorname{supp}\left(q_{2}\right)$ is downward closed in $\operatorname{supp}(q)$ and $A_{q_{2}} \leq A_{q}, A_{s_{2}}$; hence the use of lemma 9.4 is legitimate. Note that since, $\operatorname{supp}\left(q_{1}\right)$ can be an arbitrary finite subset $Y$ of $\mathcal{F} \mid Z$ we have proved the following

Claim. $\forall q \leq p_{0} \forall Y \in[\mathcal{F} \mid Z]<\omega \exists s \leq q s \| a_{Z} \notin J_{\eta(Y)}$.

Now let us finish the proof of the fact in this case of $c f(\kappa)>\omega$. Let $q \leq p, \eta<\kappa$ be such that $q \|-a_{Z} \in J_{\eta+1}-J_{\eta}$. Since $\alpha \in Z$, there is $q_{1} \leq q$ such that $q_{1} \Vdash \dot{X}_{\eta}=\check{X}$ for some $X \notin \mathcal{F} \mid Z$, but

$$
q_{1} \Vdash a_{Z} \cap a_{\dot{X}_{\eta}} \subseteq a_{X_{1}} \cup \ldots \cup a_{X_{k}} .
$$

for some $X_{1}, \ldots, X_{k}<Z$ (see definition 8.1). Now by the claim, there is $s \leq q_{1}$ such that

$$
s \| a_{Z} \notin J_{\eta\left(X_{1}, \ldots, X_{k}\right)}
$$

so $s \Vdash \eta+1>\eta\left(X_{1}, \ldots, X_{k}\right)$; hence by $\left.*\right)$ we get

$$
s \Vdash a_{Z}-a_{\dot{X}_{\eta}} \in J_{\eta+1}-J_{\eta} .
$$

This contradicts the definition of $J_{\eta+1}$. This finishes the proof in the case $c f(\kappa)>\omega$.

Now let us consider the case $\kappa=\omega$. Since $\mathcal{F}$ is a stationary coding set, we can find $Z \in \mathcal{F}$ such that

$$
\begin{gathered}
\bigcup\left\{\operatorname{supp}(p): p \in \mathcal{A}_{n}(Y), Y \in[\mathcal{F} \mid Z]^{<\aleph_{0}}\right\} \\
\cup\left\{X: \exists p \in P \exists n \in \omega p \| \check{X}=\dot{X}_{n}\right\} \subseteq \mathcal{F} \mid Z
\end{gathered}
$$

where $\mathcal{A}_{n}(Y)$ is a maximal antichain deciding the formula $\bigcup Y \in J_{n+1}-J_{n}$. Now use a similar argument as in the case $c f(\kappa)>\omega$ to show that

$$
\forall q \forall n \in \omega \exists s \leq q s \| a_{Z} \notin \dot{J}_{n} .
$$

This contradicts the fact that $P \Vdash \bigcup_{n \in \omega} J_{n}=\infty^{+}$and this completes the proof of the fact.

Now we are ready to state the main result of this section, which for $\lambda$ regular can be easily deduced from results of [N1].

Theorem 9.12. For every cardinal $\lambda$ of uncountable cofinality it is consistent that there is a countably tight compact space $X$ with a point $x$ such that $h \chi(x, X)=\lambda$ and for every clopen a such that $x \notin a$ we have $|a| \leq \omega$.

Proof. Let $A$ be the algebra $A_{\mathcal{F}}$ considered in $V^{P}$. The fact that $h \chi(\infty, X)=\lambda$ where $X$ is the Stone space of $A$ follows from Fact 9.11. The countable tightness and the remaining property follow from Fact 3.3 and Fact 9.8. 


\section{REFERENCES}

[BSV] B.Balcar, P.Simon, P.Vojtas; Refinement properties and extensions of filters in Boolean algebras; Trans. AMS. 267, 1981, pp. 265-283. MR 82k:06014

[Bal] Z.Balogh; On Compact Hausdorff Spaces of Countable Tightness; Proc. Amer. Math. Soc., 105, 1989, pp. 755-764. MR 89h:03088

[BMR] J.Baumgartner, J.Malitz, W.Reinhard; Embedding trees in the rationals; Proc. Nat. Acad. Sci. U.S.A. 67. (1970), pp. 1748-1753.j MR 47:3172

[Ba1] J.Baumgartner; Iterated Forcing; in A. Mathias, (ed); Surveys in Set Theory; Lecture Note Series 87, London Mathematical Society 1983, pp. 1-59. MR 87c:03099

[Ba 2] J.Baumgartner; Applications of Proper Forcing; in [KV], pp. 913-959. MR 86g:03084

[BK] J. Baumgartner, P. Komjath; Boolean algebras in which every chain and every antichain is countable; Fund.Math 111, 1981. pp. 125-133. MR 82j:06023

[BMS] J.Baumgartner, D.Martin, S.Shelah (eds); Axiomatic Set Theory; Contemporary Mathematics 31, 1984. MR 85g:03004

[BS] J.Baumgartner, S.Shelah; Remarks on Superatomic Boolean Algebras; Ann. Pure. Appl. Logic 33. 1987 pp. 109-130. MR 88d:03100

[De] K.Devlin; $\aleph_{1}$-trees; Annals of Mathematical Logic, 13. 1978 pp. 267-330. MR 80c:03053

[vDMR] E.vanDouwen, J.D.Monk, M.Rubin; Some Questions About Boolean Algebras; Algebra Universalis 11, 1988, pp. 220-243. MR 82a:06024

[vD] E.vanDouwen; Cardinal Functions on Boolean Spaces; in [MB], pp. 417-467. CMP 21:10

[Do] A.Dow Applications of Elementary submodels to Topology; Topology Proceedings Vol. 13, No 1, 1988, pp. 17-72. MR 91a:54003

[Fe1] V. Fedorchuk; On the cardinality of hereditarily separable compact Hausdorff spaces; Soviet Math. Dokl. 16, 1975 pp. 651-655.

[Fe2] V. Fedorchuk; A compact space having the cardinality of the continuum with no convergent sequences; Math. Proc. Camb. Phil. Soc. 81, 1977, pp. 177-181. MR 54:13827

[G] S. Grigorieff; Combinatorics on Ideals and Forcing; Annals of Mathematical Logic 3, 1971, pp. 363-393. MR 45:6614

[H] R. Hodel; Cardinal Functions I; in [KV]. MR 86j:54007

[Ju1] I.Juhasz; Cardinal Functions in Topology; Math. Centre Tracts 34, 1971 Amsterdam. MR 49:4778

[Ju2] I. Juhasz; Cardinal Functions in Topology - Ten Years Later; Math. Centre Tracts 123, 1981 Amsterdam. MR 82a:54002

[Ju3] I. Juhasz; Cardinal Functions II; in [KV]. MR 86j:54008

[Ju4] I. Juhasz; On the minimal character of points in compact spaces; Colloq. Math. Soc., János Bolyai 55, North-Holland, Amsterdam, 1993. MR 94k:54004

[Ju5] I. Juhasz; A weakening of $\mathbf{\$}$, with applications to topology; CMUC 29, 1988, pp. 767-773. MR 90d:54005

[JSz] I. Juhasz, Z.Szentmiklossy; Convergent Sequences in Compact Spaces; Proc. AMS, 116, 1992, pp. 1153-1160. MR 93b:54024

[Just] W. Just; Remarks on the altitude of Boolean algebras; Algebra Universalis, 25 (1988) pp. 283-289. MR 90d:03101

[Kop1] S. Koppelberg; Minimally Generated Boolean Algebras; Order 5, 1989, pp. 393-406. MR 90g:06022

[Kop2] S.Koppelberg; Counterexamples in minimally generated Boolean algebras; Acta Univ. Carolin. Math. Phys. 29, 1988, pp 37-46. MR 90a:06014

[Kop3] S. Koppelberg; General Theory of Boolean Algebras; in [MB], Vol I. MR 90k:06002

[Kosz1] P. Koszmider; The Consistency of $\neg C H+p a \leq \omega_{1}$; Algebra Universalis vol.27, 1990, pp. 80-87. MR 91a:03101

[Kosz2] P. Koszmider; On The Complete Invariance Property in Some Uncountable Products; Canadian Mathematical Bulletin, Vol 35 (2), 1992, pp. 221-229. MR 93g:54057

[Kosz3] P. Koszmider; Semimorasses and Nonreflection at Singular Cardinals; Annals of Pure and Applied Logic 72 (1995), 1-23. MR 96i:03043

[K] K.Kunen; Set Theory. An Introduction to Independence Proofs; North Holland, 1980. MR 82f:03001

[KV] K. Kunen, J.E.Vaughan, (eds); Handbook of Set-Theoretic Topology; North Holland, 1984. MR 85k:54001 
[Mal] V.Malykhin; A Frechet-Urysohn compactum without points of countable character; Math. Notes, 41, pp. 210-216, original in Mat. Zametki 41, 1987, pp. 365-376. MR 88g:54645

[MB] J.D. Monk, R.Bennet; (eds) Handbook of Boolean Algebras; North-Holland, 1989. MR 90k:06004

[M1] J.D. Monk; Cardinal Functions on Boolean Algebras; Lectures in Mathematics, ETH Zurich, Birkhäuser-Verlag, 1990. MR 92d:06033

[M2] J.D. Monk; Cardinal Functions on Boolean Algebras a revised version of [M1], 17 September 1992, circulated notes.

[N1] P. Nyikos; Forcing compact non-sequential spaces of countable tightness; Preprint.

[N2] P. Nyikos; Dichotomies in Compact spaces and $T_{5}$-spaces; in New Classic problems; Topology Proceedings, vol 15, pp. 201-220.

[Ra] M. Rabus; An $\omega_{2}$-minimal Boolean algebra; Trans. Amer. Math. Soc. 348, 1996, pp. 3235-3244. MR 96j:03070 1994.

[R] J. Roitman; Superatomic Boolean Algebras; in [MB] pp. 719-740. CMP 21:10

[Si] R. Sikorski; Boolean Algebras; Springer-Verlag 1964. MR 31:2178

[Shah] D.Shahmatov; Compact spaces and their generalizations; in M. Husek, J. vanMill Recent progress in General Topology; NHPC 1992, pp. 571- 640. MR 95g:54004

[Shap] B.Shapirovskii; On $\pi$-character and $\pi$-weight of compact Hausdorff spaces; Soviet math. Dokl. 16, 1975, pp. 999-1003.

[Sh1] S.Shelah; On uncountable Boolean algebras with no uncountable pairwise comparable or incomparable sets of elements; Notre Dame Journal of Formal Logic 22, 1981, pp. 301-308. MR 83d:03060

[Sh2] S. Shelah; Constructions of many complicated uncountable structures and Boolean algebras; Israel J. Math. Vol 45, No 2-3, 1983, pp. 100-146. MR 86k:06010

[Sh3] S. Shelah; Around Classification Theory of Models; Lecture Notes in Mathematics 1182, Springer-Verlag, 1986. MR 90a:03037

[Sz] Z.Szentmiklossy; S-spaces and L-spaces under Martin's axiom; Coll. Math. Soc. Janos Bolyai 23, Budapest, 1978, 1139-1145. MR 81k:54032

[Ve] D.Velleman; Souslin Trees Constructed from Morasses; in [BMS], pp. 219-241. MR 86b:03066

[W] W. Weiss; Versions of Martin's Axiom; in [KV], pp. 827-886. MR 86d:03088

[Z] W.Zwicker; $\wp_{\kappa} \lambda$ Combinatorics I: Stationary coding sets rationalize the club filter; in [BMS] , pp. 243-259. MR 86e:03046

Departmento de Matemática, Universidade de São Paulo, Caixa Postal: 66281, São Paulo, SP, CEP: 05315-970, Brasil

E-mail address: piotr@ime.usp.br 